\title{
Pulse Transition Duration Measurements and Standards at NBS - 1975 to 1988
}

W. L. Gans
N. S. Nahman
J. R. Andrews
E. E. Baldwin

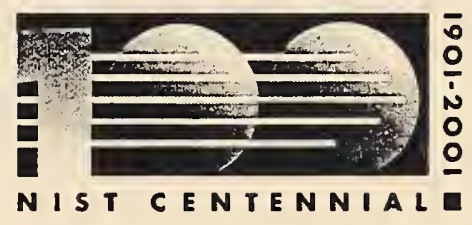

$Q C$

100

.456

N0.6550

2000 c.2 



\title{
Pulse Transition Duration Measurements and Standards at NBS - 1975 to 1988
}

\author{
W. L. Gans ${ }^{1}$ \\ N. S. Nahman ${ }^{1}$ \\ J. R. Andrews ${ }^{2}$ \\ E. E. Baldwin ${ }^{1}$ \\ ${ }^{1}$ Electricity Division \\ Electronics and Electrical Enginering Laboratory \\ National Institute of Standards and Technology \\ Gaithersburg, MD 20899-8110 \\ ${ }^{2}$ Picosecond Pulse Laboratories, Inc. \\ Boulder, CO 80306
}

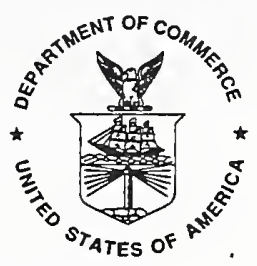

U.S. Department of Commerce Norman Y. Mineta, Secretary

Technology Administration Dr. Cheryl L. Shavers, Under Secretary of Commerce for Technology 



\section{Forward}

This NIST Interim Report (NISTIR) reports on work conducted at NBS by the authors during the period 1975 to 1988 . In large part, this work forms the foundation for the fast electrical pulse metrology services now offered at NIST ${ }^{1}$. However, for a variety of reasons, much of the work was not documented as thoroughly as is done in this note. Because more stringent documentation requirements are now in effect for all advertised NIST Calibration Services, it was deemed necessary to publish a more comprehensive treatise covering this work.

The primary pulse measurement system described in this NISTIR, the Automatic Pulse Measurement System (APMS), was replaced by a new system in 1989. The version of the APMS that is described in this IR is circa 1981. The reason for this choice is that, although additional improvements were made to the system after 1981, the 1981 version with its three measurement subsystems, was the most versatile and robust of all of the other versions, both earlier and later.

${ }^{1}$ NBS became NIST in August, 1988, with the U.S. Government's enactment of the Omnibus Trade Bill. 


\section{Table of Contents}

Foreword iii

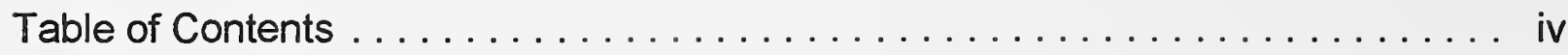

List of Figures $\ldots \ldots \ldots \ldots \ldots \ldots \ldots \ldots \ldots \ldots \ldots \ldots \ldots \ldots \ldots \ldots$

List of Tables . . . . . . . . . . . . . . . . . . . . . . .

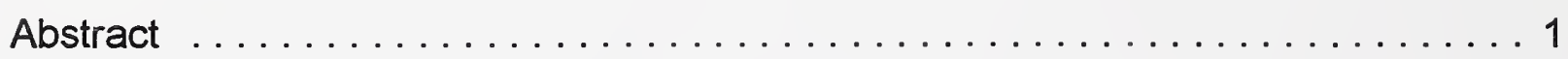

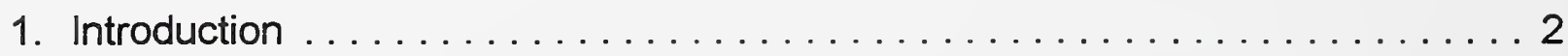

2. Pulse Transition Duration Definition $\ldots \ldots \ldots \ldots \ldots \ldots \ldots \ldots \ldots$

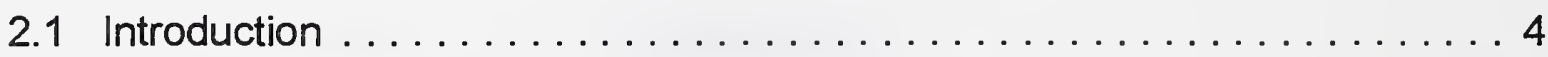

2.2 IEEE Parameters . . . . . . . . . . . . . . . . . . 4

3. Pulse Transition Duration Measurement . . . . . . . . . . . . . . . . 10

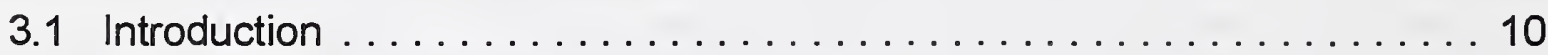

3.2 NBS APMS ........................ 10

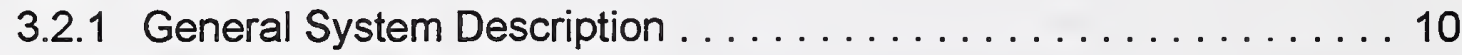

3.2.2 Sampling Oscilloscope . . . . . . . . . . . . . . . . 13

3.3 NBS Requirements for Acceptance of Step-Like Artifact

Standards for Transition Duration Calibration . . . . . . . . . . 14

3.3.1 Connectors . . . . . . . . . . . . . . . . . . . . . . . 14

3.3.2 Base and Top Characteristics ... . . . . . . . . . . . . . 14

3.3.3 Pulse Generator Synchronization . . . . . . . . . . . . . 15

3.4 Pulse Waveform Measurements . . . . . . . . . . . . . . . . . . . . 16

3.4 .1 General Remarks . . . . . . . . . . . . . . . . . . . . . 16

3.4 .2 Deconvolution . . . . . . . . . . . . . . . . . . . . . . 17

3.4.3 Pulse Generator Measurements . . . . . . . . . . . . . . . 23

3.4.4 Typical Results for Low-Pass Filter Measurements . . . . . . . . . 26

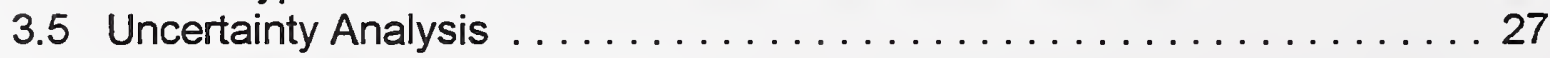

3.5.1 General Sources of Error . . . . . . . . . . . . . . . . 27

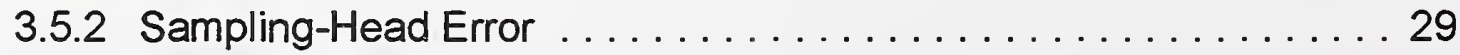

3.5 .3 System Calibration Error . . . . . . . . . . . . . . . . . 29

3.5.4 Deconvolution Error - Pulse Generator Problem . . . . . . . . . . 30

3.5.5 Deconvolution Error - Low-Pass Filter Problem . . . . . . . . . . . 33 
4. Pulse Transition Duration Transfer Standard . . . . . . . . . . . . . . . 49

4.1 Introduction . . . . . . . . . . . . . . . . . . . . 49

4.2 Objective and Concept . . . . . . . . . . . . . . . . . 49

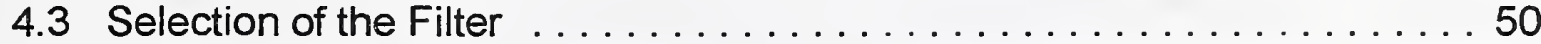

4.4 The Filter Model . . . . . . . . . . . . . . . . . . . . . 50

4.5 Definition and Prediction of the Available-Pulse (Waveform) . . . . . . . 53

4.6 Hardware ................................ 55

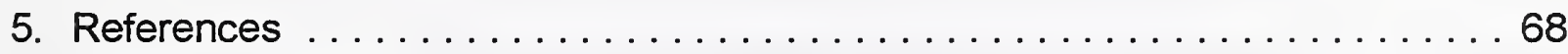

6. Appendix 1: NBS Pulse Transition Duration Transfer Standard

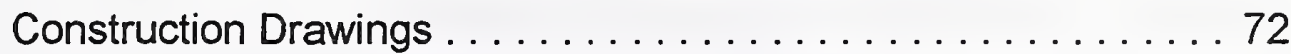

7. Appendix 2: NBS Pulse Transition Duration Transfer Standard Temperature Controller .......................86 86

8. Appendix 3: NBS Pulse Transition Duration Transfer Standard Automatic Tunnel Diode Bias Supply . . . . . . . . . . . . . 101 


\section{List of Figures}

Figure 2.1 A single pulse $[4,5] \ldots \ldots \ldots \ldots \ldots \ldots \ldots \ldots \ldots$

Figure 2.2 Graphical determination of the occurrence density $[3,6]$. (A) - Pulse waveform with superimposed grid. B - Probability density histogram. . . . . . . . . 9

Figure 3.1 The Automatic Pulse Measurement System (APMS) . . . . . . . 34

Figure 3.2 The APMS minicomputer subsystem $\ldots \ldots \ldots \ldots \ldots \ldots \ldots$

Figure 3.3 HP-1430A 28 ps sampling head step response; vertical scale factor is $111.1 \mathrm{mV} /$ div and time scale factor is $25.6 \mathrm{ps} / \mathrm{div}$; response bias $=-1.76 \mathrm{~V} \ldots \ldots \ldots . \ldots 36$

Figure 3.4 The step response and probability density histogram of the model for the Hewlett-Packard Model 1430B sampling head used in the NBS APMS

Figure 3.5 HP-1430A 28 ps sampling head impulse response; vertical scale factor is arbitrary and time scale factor is $25.6 \mathrm{ps} / \mathrm{div}$; response bias $=-1.76 \mathrm{~V} \ldots \ldots \ldots \ldots \ldots \ldots$

Figure 3.6 The impulse response and probability density histogram of the model for the Hewlett-Packard Model 1430B sampling head used in the NBS APMS .

Figure 3.7 Block diagram illustrating the convolution and deconvolution problems

Figure 3.8 An actual physical measurement system. The actual variables, $x(t), h(t)$, and $y(t)$ differ from the conceptual ones by the respective departures $n_{x}(t), n_{h}(t)$, and $n_{y}(t)$

Figure 3.9 The pulse-generator measurement system. In principle, the output $y(n \Delta t)$ is equal to the convolution of the pulse being measured $x(n \Delta t)$ with the impulse response of the measurement system $h(n \Delta t)$ 
Figure 3.10 A pulse generator problem. (A) The measured output waveform $y(n \Delta t)$ from an NBS 100 ps transition duration standard; TD $=103.6 \mathrm{ps}$. (B) The sampler estimated step response $h_{0}(n \Delta t)$; TD $=20.85 \mathrm{ps}$. (C) The deconvolved estimate $\left(\gamma_{0}=1.35 \times 10^{5}\right)$ of the true output waveform from the standard $\left[d_{x}(n \Delta t)\right]_{y} ; T D=97.4$ ps. $N=512$ points, vertical scale factors for $(A)$ and $(C)$ are $50 \mathrm{mV} / \mathrm{div}$, for $(B)$, $200 \mathrm{mV} / \mathrm{div}$, and time scale factor for all is $200 \mathrm{ps} / \mathrm{div}$.

Figure 3.11 The low-pass filter measurement system. CC denptes the connector pair. The measured waveforms are shown as $x(n \Delta t)$ and $y(n \Delta t)$. In principle, the response $y(n \Delta t)$ is equal to the convolution of the reference waveform $x(n \Delta t)$ with the impulse response $h(n \Delta t)$ of the filter being measured

Figure 3.12 A low-pass filter problem. (A) The measured reference waveform $x(n \Delta t) ; V_{\text {pk-pk }}=25.8 \mathrm{~V}$. (B) The measured response waveform $y(n \Delta t)$ for a $3.5 \mathrm{GHz}$ low-pass filter; $\mathrm{V}_{\mathrm{pk} \text {-pk }}=23.7 \mathrm{~V}$. For both waveforms, $\mathrm{N}=512$ points, time scale factor is $1 \mathrm{~ns} / \mathrm{div}$, and the number of waveform ensemble averages is 100

Figure 3.13 A low-pass filter problem. (A) The deconvolved estimate $\left(Y_{0}=9 \times 10^{8}\right)$ of the $3.5 \mathrm{GHz}$ low-pass filter impulse response $\left[d_{h}(n \Delta t)\right]_{\text {yo }}$. (B) The estimated step response of the filter (integral of the impulse response); TD $=124.8 \mathrm{ps}$. Vertical scale factor for $(A)$ is arbitrary and for (B) is $200 \mathrm{mV} /$ div; time scale factor is $1 \mathrm{~ns} / \mathrm{div}$ for both waveforms

Figure 3.14 A pulse generator problem. (A) The spectrum magnitude $\left|D_{x}(k \Delta \Omega)\right|$ of the deconvolved estimate of the true output waveform $d_{x}(n \Delta t)$ with no filtering, $Y=0$. (B) The spectrum magnitude of the deconvolved estimate of the true output waveform with optimal filtering, $Y=1.35 \times 10^{5}$. For both spectra, vertical scale factor is $10 \mathrm{~dB} /$ div and frequency scale factor is $12.5 \mathrm{GHz} /$ div. (See Fig 3.10 for associated timedomain waveform plots.). 
Figure 3.15 A low-pass filter problem. (A) The spectrum magnitude $\left|D_{h}(k \Delta \Omega)\right|$ of the deconvolved estimate of the impulse response $d_{h}(n \Delta t)$ of the $3.5 \mathrm{GHz}$ low-pass filter with no filtering; $y=0$. (B) The spectrum magnitude of the deconvolved estimate of the impulse response of the $3.5 \mathrm{GHz}$ filter with optimal filtering; $\gamma=9.0 \times 10^{8}$. For both spectra, vertical scale factor is $10 \mathrm{~dB} / \mathrm{div}$ and frequency scale factor is $1.25 \mathrm{GHz} /$ div. (See Fig 3.13 for associated time-domain waveform plots.)

Figure 4.1 Typical step response $f(t)$ of a low-pass filter having a sharp cut-off frequency

Figure 4.2 Commercial 2 ns transition duration standard. Note the small steps on the leading edge. The lower curve shows the leading edge on a faster time scale. .

Figure 4.3 Step response of a typical coaxial cable with skineffect loss. Note the long "dribble-up", that is, the slow approach to the final value, $\mathrm{E}$.

Figure 4.4 Debye-type dielectric function . . . . . . . . . . . . . . . 59

Figure 4.5 Per-unit-length equivalent circuit of a transmission line with Debye-type dielectric $\ldots \ldots \ldots \ldots \ldots \ldots \ldots \ldots \ldots$

Figure 4.6 Step-response transition duration versus Debye-

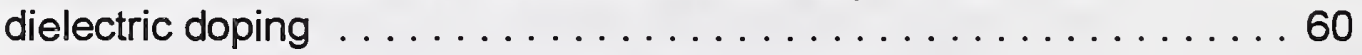

Figure 4.7 Unit-step response of a Debye-dielectric, coaxial low-

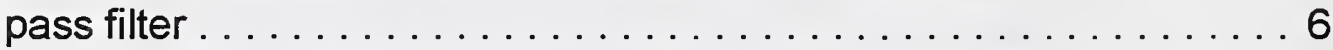

Figure 4.8 (A) The complete model of the Debye-dielectric filter including the connectors $N_{3}$ and $N_{4}$. (B) The equivalent circuit for connector $\mathrm{N}_{3}$. (C) The equivalent circuit for connector $\mathrm{N}_{4}$

Figure 4.9 (A) The tunnel-diode transition generator operating into a $50 \Omega$ load with the load voltage being observed by the oscilloscope system, $H(s)$. (B) The tunnel-diode transition generator driving the lowpass filter with the low-pass filter load voltage being observed by the oscilloscope system, $H(s)$. 
Figure 4.10 Measured and deconvolved waveforms from the

(a) tunnel diode and the (b) $50 \mathrm{ps}$, (c) $100 \mathrm{ps}$, and

(d) 200 ps low-pass filters.

Figure 4.11 Available reference pulse into a $50 \Omega$ load from The TD/100 ps filter combination, $e_{\mathrm{a}}(t)$.

Figure 4.12 Overlay of a predicted 100 ps reference pulse, $e_{a}(t)$, the actual measured waveform (dotted line), $e_{\mathrm{am}}(t)$, and their resultant difference, $\Delta(t)$. The waveforms are aligned so that their $50 \%$ values coincide with each other.

Figure 4.13 (A) Photograph of the NBS reference waveform pulse generator. (B) Generator shown with associated temperature controller, tunnel-diode pulse generator (attached to rear APC-7 connector), and APMS dualchannel sampler (attached to front APC-7 connector). 


\section{List of Tables}

Table 3.1 Nominal values of pulse generator peak-to-peak time jitter, $J$, acceptable to NBS . . . . . . . . . . . . . . 16

Table 4.1 As-built typical filter physical parameters . . . . . . . . . . . 57 


\title{
PULSE TRANSITION DURATION MEASUREMENTS AND STANDARDS AT NBS -- 1975 TO 1988
}

\author{
Norris S. Nahman \\ National Institute of Standards and Technology (Ret.) \\ Boulder, CO 80303 \\ James R. Andrews \\ Picosecond Pulse Labs, Incorporated \\ Boulder, CO 80306 \\ (formerly with NBS -- 1967-80) \\ William L. Gans \\ National Institute of Standards and Technology (Ret.) \\ Gaithersburg, MD 20899 \\ E. E. Baldwin \\ National Institute of Standards and Technology (Ret.) \\ Boulder, CO 80803
}

\begin{abstract}
The techniques employed by NBS from the mid 70s through 1988 for the calibration of the pulse transition duration (TD) of a fast, step-like, electrical pulse generator are described along with the artifact requirements imposed on the pulse generator. The fundamental definitions, and measurement and generation methods relevant to the calibration of pulse transition duration are presented. Subjects discussed include the IEEE Pulse Standards 181-77 and 194-77, deconvolution as applied in (1) time-domain waveform measurements of a pulse and in (2) network impulse response characterization, and the NBS 50ps, 100ps, and 200 ps Transition Duration Transfer Standards which are based upon a tunnel diode step-like generator (TD about $20 \mathrm{ps}$ ), and Debye liquid-dielectric coaxial line filters. The Appendices contain electronic circuit diagrams and mechanical drawings for the Transition Duration Transfer Standards.

Key words: deconvolution, impulse response, pulse, rise time, step response, time domain metrology, transfer standards, transition duration, waveform.
\end{abstract}




\section{Introduction}

Pulse transition duration (formerly called pulse rise or fall time) is a parameter that most technical people understand intuitively. It is defined as the (time) interval in which a pulse changes in magnitude from one value to another; usually, the magnitude levels are defined as the $10 \%$ and $90 \%$ values of the pulse amplitude (100\% level). However, pulse transition duration is one of the more difficult parameters to measure precisely due to the difficulty of determining the following factors:

(1) The fidelity of the measured or observed waveform with respect to the physical or actual pulse.

(2) The waveform baseline and topline values, i.e., the $0 \%$ and $100 \%$ levels whose difference define the waveform amplitude.

(3) The absolute calibration of the waveform magnitude and time scales.

As it was implied above, the terms pulse and waveform are not interchangeable and are defined in the IEEE Standard 194[4], and identically defined in the IEC Publication No. 469-1 [5]. Pulse is defined as the physical modifications of state, while waveform is reserved for the observed or measured shape of the pulse. Clearly, the pulse and its corresponding waveform (through measurement) will differ; in other words, the measurement or observation process yields a waveform that is not an identical replica of the pulsed physical state, that is, the pulse.

Consequently, pulse transition duration is that transition duration belonging to the pulse while waveform transition duration belongs to the waveform. To be able to determine the pulse transition duration corresponding to a given (observed) waveform requires the application of a deconvolution method to remove the effects of the measurement instrument. Thus, the pulse transition duration must always be inferred from the observable waveform through a deconvolution process.

The objective of this technical note is to present the fundamental definitions, and measurement and generation methods relevant to the calibration of pulse transition duration, and in particular, to present the methods used and the artifact requirements imposed by NIST in the calibration of pulse transition duration. To such an end, this Technical Note is organized into four chapters and three appendices. The first chapter is the present introduction.

Chapter 2 is concerned with the definition of pulse (waveform) parameters and the algorithms (means) for establishing their values. 
Chapter 3 presents the NBS methods for the calibration of pulse transition duration. Also described are the NBS requirements for pulse generators and networks in order for them to be acceptable for NBS calibration (connectors, pulse shape, etc.).

Chapter 4 discusses reference pulse generators and describes the NBS pulse transition duration transfer standards which employ the Debye-dielectric relaxation phenomena to provide a step-like pulse having a specified transition duration and a specified source impedance.

Appendix 1 contains the construction drawings for the NBS pulse transition duration transfer standards while Appendix 2 contains the theory, calibration and adjustment procedures, and schematics for the transfer standards' associated temperature controllers. Finally, Appendix 3 contains the theory, schematics, and parts list for the NBS Automatic Tunnel Diode Bias Supply. 


\section{Pulse Transition Duration Definition}

\subsection{Introduction}

The application of pulse time-domain measurements continues to be broadly developed throughout the technical community due to the continuing expansion of digital methods and pulse techniques in many areas including communications, fusion energy systems, instrumentation, pulsed electromagnetic fields, time-domain reflectometry and transmission measurements, and time-domain spectroscopy. From about 1965 to 1980 digital computer systems evolved from the large computer to the minicomputer and subsequently to the microprocessor. This evolution has led to automated time-domain measurement systems capable of acquiring, processing, and analyzing pulse signals in a sophisticated manner far beyond the limited methods applied in the past to an oscillographic display.

The field of pulse measurements is one area of measurement science that has historically been identified as having poor accuracy and precision. Aside from the instrumentation difficulties, a major cause of this had been the lack of precise standard terms and definitions to characterize the infinite variety of complicated pulse shapes that are encountered in actual practice. In comparison, the signal shapes encountered in dc and frequency-domain measurements are extremely simple and mathematically definable, namely a straight-line and a sinusoidal curve, respectively. In 1967 the Institute of Electrical and Electronic Engineers (IEEE) recognized the need for improved pulse standards and thus established the Subcommittee on Pulse Techniques (SCOPT) within the High Frequency Instrumentation and Measurement Society. SCOPT was charged with the task of completely revising the IEEE Pulse Standards 181 and 194 [1,2]. The International Electrotechnical Commission (IEC) joined with the IEEE in this endeavor, and both organizations individually issued the (same) revised standards during the period 1974-77 [3-6].

Use of these new IEEE/IEC Pulse Standards in conjunction with automated pulse measurement systems now allows consistent and repeatable measurements to be made on pulses and suitably defined pulse parameters. Consistency and repeatability are fundamental to fostering measurement agreement between laboratories.

\subsection{IEEE Pulse Parameters}

For clarity, it is appropriate at this point in the discussion to state a few definitions from the IEEE/IEC pulse standards. The term pulse is defined as pertaining to the modification 
of physical state while the term waveform is used when referring to the observed, measured, or modeled shape of the pulse. Clearly, the pulse and its waveform do differ; in other words, the measurement or modeling process always yields a waveform that is not an exact replica of the pulsed physical state. Accordingly, the terms pulse and waveform must also be used as adjectives. As an example, for the case of pulse area and waveform area, the area under the curve describing the pulse is being denoted as inherently different from that under the waveform curve. Also acceptable is such usage as pulse waveform area where the adjective pulse is redundant because waveform is the measurement system or modeling entity corresponding to the physical pulse.

The difference between two values of time is defined in the standards as duration because the word "duration" denotes extent in time while "width," an often-used misnomer, denotes extent in space. Prior to the introduction of the new standards, for a simple pulse in which the modifications in state occur in a smooth quasi-increasing and/or decreasing manner, the terms rise time and fall time were used to denote the off-to-on and on-to-off states, respectively. The term rise was used for the first change of state even when the pulse or waveform fell to its on-state. In the IEEE/IEC terminology, the terms first transition duration and last or second transition duration are used. Note that for a waveform that does not turn on and off in a simple monotonic manner, several transition durations may be needed to characterize the waveform's leading/trailing edges.

It is common practice to describe a waveform transition in terms of three values in time, for example, the times at which the waveform crosses the $10 \%, 50 \%$, and $90 \%$ pulse amplitude levels. These three levels are commonly used, but in general other levels may be more appropriate for a given situation. Accordingly, the three levels are denoted as proximal, mesial, and distal levels, respectively. Proximal, mesial, and distal are English language words denoting nearest, toward the middle, and farthest, respectively. They are used in the context of being nearest, toward the middle, and farthest from the waveform baseline. In a given application, if these levels are not numerically defined, they are to be assumed to be the $10 \%, 50 \%$, and $90 \%$ levels, respectively. A transition duration is defined as the difference in time between the proximal and distal points of a given transition. A waveform duration is defined as the difference in time between the first and last mesial points of the first and last transitions. Fig 2.1 is reproduced from the 1977 IEEE Pulse Standard and illustrates these definitions for a single pulse.

The parameters that must first be determined are the base magnitude and top magnitude whose difference defines the waveform amplitude. All of the other lines and points of Fig 2.1 are obtained directly from their definitions once the base and top magnitudes are established. Thus, obtaining parameters such as pulse amplitude, 
transition duration, pulse duration, etc., is a definable process once the base and top magnitudes have been determined.

Until recently, the determination of the base and top magnitudes has been difficult at best. This is due to the fact that most real pulses are not ideal, trapezoidal pulses, but are distorted in the pulse base and top regions. The actual determination of the base and top magnitudes has relied upon human judgment in determining the most probable base and top lines from a visual inspection of an oscilloscope display or waveform photograph. Early attempts at automating pulse measurements used either the minimum-and-maximum waveform values or the (turn-on) first-and-last waveform values as the base and top magnitudes, respectively. This was done primarily because such values were easily specified or measured. However, the pulse parameter values obtained using such methods often did not agree with those produced by a human observer using other criteria and subjective judgment.

The IEEE 181-1977 (IEC 469-1) standard on pulse measurement and analysis [3,6] describes four algorithms that can be used to determine the base and top magnitudes. The maximum/minimum values and the first/last data points are two of the algorithms. The other two use either the mean or the mode of the pulse waveform occurrence density distribution, or histogram, as is illustrated in Fig 2.2.

The occurrence density distribution is obtained by superimposing a rectangular grid of elemental dimensions $\Delta t$ and $\Delta m$ over the pulse waveform, Fig 2.2A. The probability density histogram is then determined by counting the number of elementary rectangles through which the pulse waveform passes for each vertical element of height $\Delta m$. The result, Fig 2.2B, is a bimodal histogram in which the base and top histograms can be identified. The development of an accurate histogram can be a very tedious task if it is done manually from recorded data. However, it is a simple task for an automated measurement system that is based upon high resolution analog to digital converters and a digital computer for analysis and control.

NBS chose to use the mode of the histogram algorithm for establishing the base and top magnitudes of a pulse waveform having appreciable top and base durations. The mode of the histogram is the value of the histogram (distribution) that occurs most frequently in the distribution, that is, the maximum value of the histogram. As pointed out in the IEEE Standard 181-1977, the histogram mode values for base and top magnitudes are compatible to those obtained with visual observation and analysis by an operator.

Depending on the situation, it is not always necessary or desirable to use the same algorithm for both the base and top magnitude $[3,6]$. For example, the peak magnitude 
algorithm is best suited to the analysis of pulse waveforms with tops of negligible or relatively short duration, while the histogram mode would be used for a base of significant duration $[3,6]$. 


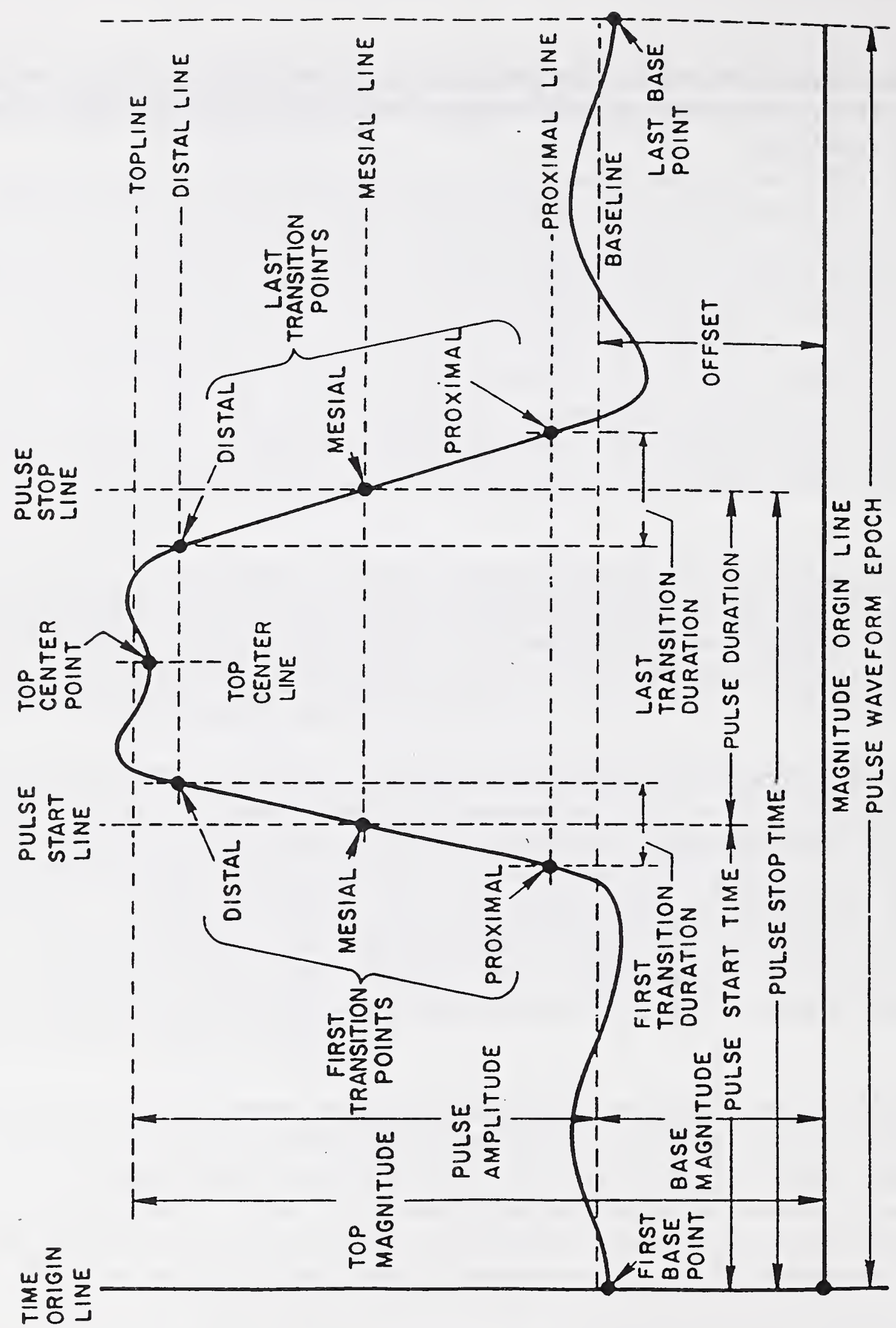

Figure 2.1 A single pulse $[4,5]$. 

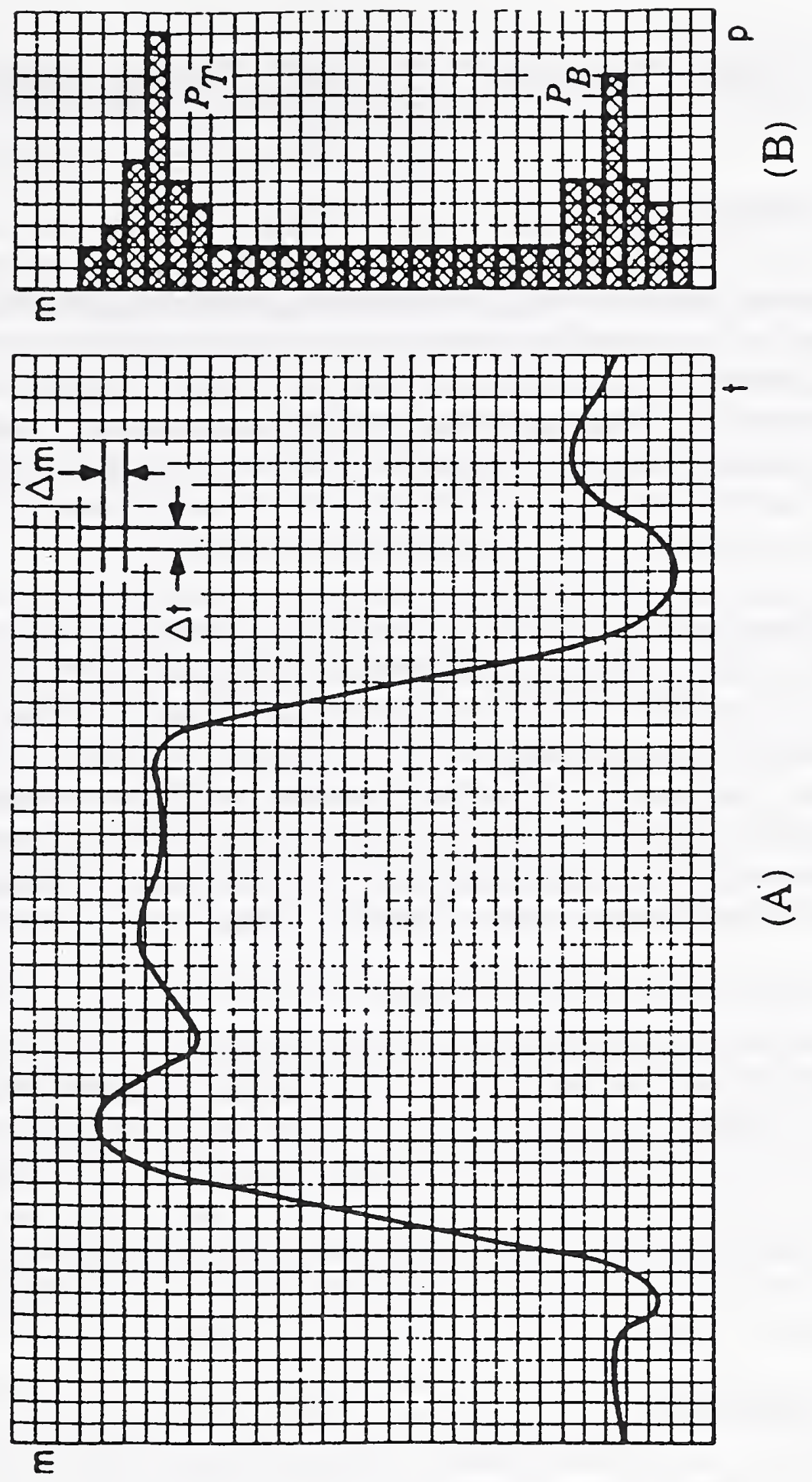

Figure 2.2 Graphical determination of occurrence density [3,6]. (A) - Pulse waveform with superimposed grid. (B) -- Probability density histogram. 


\section{Pulse Transition Duration Measurement}

\subsection{Introduction}

In general, a pulse transition-duration measurement is performed in three steps. First, the waveform corresponding to the pulse is determined using a calibrated measurement system. Second, the pulse shape is obtained (estimated) by a deconvolution process. For a pulse generator, the deconvolution employs the measurement system impulse response and the measured pulse waveform; for a two-port network, the measured reference and insertion waveforms are used. Third, the pulse transition duration is calculated using the pulse shape obtained from the deconvolution operation.

For the purposes of fostering measurement agreement between various laboratories involved in time-domain measurements of pulses and their parameters, the National Bureau of Standards limited its advertised pulse transition duration calibration service to the calibration of artifact step-like pulse standards meeting certain requirements. Pulse generators and two-port networks acceptable by NBS for calibration were required to employ APC-7 coaxial connectors (or other connectors with appropriate adapters attached) and to meet certain top and base flatness requirements. Pulse generators were also required to meet certain synchronization and triggering requirements.

The NBS transition duration calibration was performed using the NBS Automatic Pulse Measurement System (APMS). This system was a sampling oscilloscope that was interfaced with and controlled by a minicomputer. The calibration of the APMS, pulse measurements, and data reduction, were all carried out using the computation ability of the minicomputer to implement control, data acquisition, and data reduction algorithms.

Discussed below are the APMS, criteria for a pulse generator or a two-port network to be accepted by NBS for calibration, pulse waveform measurement, pulse shape estimation and transition duration calculation, and error analysis.

\subsection{NBS APMS}

\subsubsection{General System Description}

The automatic pulse measurement system (APMS) was used from 1975 to 1988 in the NBS Electromagnetic Waveform Metrology Group to measure and analyze the waveforms of electrical and optical signals (pulses) [7-11]. For the purposes of this Technical Note, 
the APMS and on-going subsystem development work are described as of the year 1981 . Also, for the sake of completeness in describing the APMS in its 1981 form, some mention of optical pulse measurement capability will be made.

The APMS may be considered to consist of three major contributing components; a minicomputer subsystem, a set of measurement subsystems, and a knowledgeable operator. There were three measurement subsystems in existence or being developed for the APMS in 1981, with the capability of adding more subsystems as the need arose. As indicated in Fig 3.1, the three measurement subsystems were a low-light-level television camera/video analyzer (top four boxes in Fig 3.1), a linear photodiode array (lower-left two boxes of Fig 3.1), and a wideband dual-channel sampling oscilloscope (lower-right two boxes of Fig 3.1).

The television camera subsystem was primarily intended for the acquisition of the display of single-shot optical or electrical signals such as might be displayed on the fluorescent screen of an optical-streak camera or an electrical traveling-wave oscilloscope. The camera was interfaced to the APMS through a video-disk memory and a video analyzer such that the video-image data from a single recorded television frame could be digitized and read into the computer memory at a rate of $30 \mathrm{~Hz}$. The time-domain resolution of this subsystem input was limited fundamentally by the time-domain resolution of the imaging instrument. In the case of an optical-streak camera, for example, it was between 1 ps and 10 ps, and, for an electrical traveling-wave oscilloscope, between 50 ps and $100 \mathrm{ps}$.

In the second measurement subsystem, the linear photodiode array consisted of an array of 1024 photodiodes with an equal spacing of $24.5 \mu \mathrm{m}$ between diodes. In this configuration, the first 1000 photodiodes were interrogated and read into 1000-point 3-digit binary-coded-decimal (BCD) memory via an analog-to-digital converter (ADC) and stored for subsequent transfer to the APMS main-computer memory. This input was primarily intended for two photon-fluorescence optical pulse measurements but could be used in any linear imaging scheme.

Of specific interest in the present Technical Note is the remaining operational measurement subsystem used for repetitive electrical pulse measurements. The measurement instrument was a Hewlett-Packard ${ }^{2}$ sampling oscilloscope consisting of a Model 181AR mainframe, an NBS-modified Model 1811A sampling time base and vertical

${ }^{2}$ The identification of commercial instruments and/or components in this document does not imply endorsement by the National Institute of Standards and Technology, nor does it imply that the instruments/components identified are necessarily the best available for the purpose. 
amplifier plug-in, and a Model $1430 \mathrm{~B}$ sampling head. The $1430 \mathrm{~B}$ was a wideband, (dc$18 \mathrm{GHz}$ ), $20 \mathrm{ps}$, dual-channel, $50 \Omega$ feed-through sampling head. With this subsystem, the APMS was essentially a computer-controlled sampling oscilloscope. This system input was limited to the acquisition of repetitive (detected) optical or electrical signals but, unlike the first two inputs, was a true electronic input with no intermediate optical-imaging stage. The available sweep speeds ranged from $10 \mathrm{ps} / \mathrm{cm}$ to $5 \mu \mathrm{s} / \mathrm{cm}$ and the sampler voltage range was $\pm 1 \mathrm{~V}$. Optical pulses were measured in this configuration via an optical/electrical transducer such as a fast photodiode or photoconductor.

As shown in Fig 3.1, all three input subsystems were interfaced to the minicomputer subsystem through an Analogic Model 5800 modular data acquisition and distribution system containing a 14-bit analog-to-digital converter (ADC) and a 14-bit digital-to-analog converter (DAC). Thus, the data input to the computer could be thought of as being points in a 16384 -point by 16384 -point grid or plane. Therefore, the resolution of the computer input was better than 1 part in $10^{4}$ yielding a dynamic range in excess of $80 \mathrm{~dB}$. However, for practical measurements in the presence of system and external noises, this dynamic range could not be achieved. For the sampling-oscilloscope system input, for example, a signal/noise ratio of $(40-50) \mathrm{dB}$ was typical.

The minicomputer subsystem, a block diagram of which is shown in figure 3.2 , was composed of a Data General NOVA-1200 minicomputer mainframe, a flexible disc-drive unit, a Tektronix 4010 storage cathode-ray-tube (crt) terminal with a hardcopy unit and a high-speed paper-tape reader and punch. The minicomputer mainframe contained a 16bit-word-length central processing unit (cpu), 32k words of nonvolatile core memory, peripheral interfaces and a hardware floating-point-number processor. The latter reduced lengthy calculation times such as for the fast Fourier transform or time-domain convolution/deconvolution by a factor of about 50 . The flexible disc mass storage unit provided an additional $120 \mathrm{k}$ words of on-line memory for data and program storage. The storage crt terminal, interfaced through a modified teletype port, ran in excess of $100 \mathrm{k}$ baud. It operated in both an alphanumeric and graphic mode allowing a wide variety of information output formats. The hard-copy unit, either under program control or manually, provided a standard-page-size paper copy of whatever was displayed on the terminal screen.

Normally, the maximum number of waveform data points was restricted to 2048 points in order to insure that the non-volatile memory capacity of $32 \mathrm{k}$ words was adequate for the total memory requirements for data acquisition, computation, input/output control, etc.

The system software was both sophisticated and versatile. Briefly, this disc operating system contained everything necessary for system operation with Fortran IV. The 
compiler, assemblers, loaders, editors, and libraries were all contained on flexible discs as was an extensive package of diagnostic programs. From the user's or programmer's point of view, practically all programming, including graphics, was done in Fortran. The one exception was that programs written to control the ADC and DAC had to be written by the programmer in assembly language as called Fortran subroutines.

The commercially available HP-181AR sampling oscilloscope was used in the APMS although NBS modifications were necessary to interface it with the minicomputer. The minicomputer drove the time-axis sampling position in the time base through the voltage output of the 14-bit DAC. After setting the sampling-time position, the oscilloscope trigger circuit was enabled, allowing the sampling of a new value on the waveform. The oscilloscope then provided an analog voltage proportional to the waveform sample value, along with a conversion-command pulse to the 14-bit ADC. Considerable digital logic circuitry was also added in the interface to prevent the acquisition of false data. The factory-installed sampling oscilloscope display smoothing-filter circuit was replaced with a more effective analog signal averager. The normal sampler tangential noise level was about $10 \mathrm{mV}$. With the NBS signal averager the noise was reduced to less than $1 \mathrm{mV}$ with 100 averages.

The minicomputer was a vital element in the operation of the APMS. It served many functions including: (1) measurement system controller, (2) data acquisition, (3) data storage, (4) digital averaging to improve signal to noise ratio, (5) computation of the desired pulse parameters using various algorithms, (6) computation of transforms and their inverses such as the fast Fourier transform and the $Z$ transform, and (7) presentation of the results in a useful tabular or graphical form to the operator via the crt terminal. The APMS could also be used as a network analyzer to measure the frequency-domain scattering parameters $\left(S_{i j}(\omega)\right)$ of $r f$ and microwave networks $[8-11,13,14]$. This capability was reflected in a NBS-advertised Special Test Service for network time-domain impulse response/step response and frequency-domain transfer function for microwave networks.

\subsubsection{Sampling Oscilloscope}

The NBS APMS sampling oscilloscope used a HP-1430B remote sampling head. The nominal $3 \mathrm{~dB}$ bandwidth was dc to $18 \mathrm{Ghz}$ and the input impedance was $50 \Omega$. It was built in a feed-through configuration, and the input and output connectors were $50 \Omega$, precision, $7 \mathrm{~mm}$, APC-7 connectors. In normal useage, the output was terminated with a $50 \Omega$, precision, APC-7 termination. The sampler dynamic range was $\pm 1 \mathrm{~V}$.

In 1975-6, an NBS guest worker, Sedki Riad developed a detailed physical and electrical model for the HP-1430A sampling head [12]. The 1430A had a nominal $3 \mathrm{~dB}$ 
bandwidth of $12 \mathrm{GHz}$ and a nominal step-response first transition duration of $28 \mathrm{ps.} \mathrm{Using}$ the model, computer simulation results were in excellent agreement with experimental data [12,23,24]. When the $18 \mathrm{Ghz}, 20 \mathrm{ps}, \mathrm{HP}-1430 \mathrm{~B}$ sampler became available and was introduced into the APMS, NBS studies revealed that its construction was very similar to that of the 1430A. Thus, portions of Riad's $12 \mathrm{Ghz} 1430 \mathrm{~A}$ model were scaled to $18 \mathrm{GHz}$ in order to obtain a modeled estimate for the 1430B. Based upon studies made in the late 70 's, the 1981 NBS uncertainty statement for the HP-1430B 20 ps sampler that resulted was 20 ps ( +3 ps and -5 ps).

The step response of the 28 ps 1430 A sampling head [12], Fig 3.3, was scaled to correspond to a sampling head possessing a step-response transition duration of $20 \mathrm{ps,}$ Fig 3.4. Also, corrections were made to account for the (slight) structural continuity differences between the two sampling heads. The time scale for the 20 ps unit was chosen as $200 \mathrm{ps} /$ division in order to display the small variations about the $100 \%$ level due to reflections from various known internal discontinuities. The corresponding impulse responses are shown in Figs 3.5 and 3.6, respectively.

\subsection{NIST Requirements for Acceptance of Step-Like Artifact Standards for Transition Duration Calibration}

\subsubsection{Connectors}

In general, the required pulse signal-path connector was the APC-7 connector. If a given step-like pulse generator had a pulse signal-path connector other than the APC-7 connector, the connector was required to be connected to an APC-7 adapter. Similarly, if a two-port network had connectors other than the APC-7 connectors, the two connectors were required to be connected to APC-7 adapters. The NBS calibration was for the customer-supplied total artifact standard which included any required APC-7 adapters. Furthermore, a customer-supplied cascade of adapters having a configuration which yielded APC-7 input and output connections could be calibrated as a two-port network.

\subsubsection{Base and Top Characteristics}

The step-like pulse or network step response were required be such that the base and top magnitudes could be determined by the two modes (maximum values) of the bi-modal histogram. Also, the required time epoch for clear definition of the two modes was nominally about ten to one hundred times the nominal first transition duration. These requirements excluded step-like pulses or step responses containing slowly varying 
components which produced ramp functions in the base and top regions of the step-like pulse or step response.

\subsubsection{Pulse Generator Synchronization}

There are three classifications for pulse generators from the viewpoint of synchronization of their output to a pulse measurement system. The classifications are:

1. Triggerable - produces a pulse after the application of an external triggering pulse (command).

2. Pretrigger Output - produces a trigger pulse for external use which precedes the pulse output by a time interval great enough to allow adequate measurement of a desired pulse.

3. Free-running aperiodic-occurrence of the pulse is not under external command nor periodic.

For a triggerable generator, the trigger pulse required to trigger the generator was subject to the following NBS requirements: a pulse having an amplitude equal to or less than $0.5 \mathrm{~V}$ and a first transition duration equal to or less than $1 \mathrm{~ns}$.

For a pretrigger-output type of generator, the pretrigger output pulse was subject to the following NBS requirements: a pulse having an amplitude equal to or greater than $1.5 \mathrm{~V}$, a first transition duration equal to or less than $1 \mathrm{~ns}$, and a nominal pretrigger time advance of $70 \mathrm{~ns}$.

For both types of generators, smaller and/or slower triggering pulses would degrade the resultant measurement; also, the generators were subject to the NBS jitter limitations given in Table $3.1^{3}$.

${ }^{3}$ The jitter limitations could be relaxed if the jitter components could be deconvolved from the observed data. See Section 3.5.1. 
Table 3.1

Nominal values of pulse generator peak-to-peak jitter, $J$, acceptable to NBS.

\begin{tabular}{|c|c|c|}
\hline \multirow{2}{*}{$\begin{array}{c}\text { Nominal Pulse } \\
\text { Transition Duration, TD }\end{array}$} & \multicolumn{2}{|c|}{ Nominal Acceptable Jitter } \\
\cline { 2 - 3 } & $\mathrm{J}$ (peak-to-peak) & $\%$ of TD \\
\hline $20 \mathrm{ps}$ & $10 \mathrm{ps}$ & 50 \\
\hline $50 \mathrm{ps}$ & $15 \mathrm{ps}$ & 30 \\
\hline $100 \mathrm{ps}$ & $20 \mathrm{ps}$ & 20 \\
\hline $200 \mathrm{ps}$ & $20 \mathrm{ps}$ & 10 \\
\hline $500 \mathrm{ps}$ & $50 \mathrm{ps}$ & 10 \\
\hline
\end{tabular}

The free-running aperiodic type of generator required the insertion of an NBS signal splitter and pulse delay line into the pulse signal path to provide synchronization. Because of the inherent effects of these additional components in the pulse signal path, the calibration accuracy in most cases was less than that achievable for a comparable pulse signal from a triggerable or pretrigger type of generator.

In particular, because a nominal 70 ns pulse delay was required, a transmission line of suitable length would distort the pulse generator output to a further degree than that introduced by the pulse splitter [25]. In some calibrations a low joule-loss semi-solid dielectric transmission line could be used [26]; however, the pulse distortion was quite severe due to the transmission line dispersion (phase transmission distortion). For pulses having small transition durations (say, 150 ps or less), a superconducting coaxial transmission line was required. A superconducting coaxial transmission-line assembly (70 ns delay) developed by NBS for such delay applications possessed an estimated step response transition duration of 15 ps, based upon measurement experiments [27].

\subsection{Pulse Waveform Measurements}

\subsubsection{General Remarks}

In Section 3.1 it was pointed out that there are two situations to be considered in which it is desired to determine the transition duration of a step-like pulse signal. First, there is the case of a pulse generator that generates a step-like pulse, where the term step-like 
means that the transition duration of the pulse is not zero; if it were zero, the pulse would be a perfect step. The second case is that of the step response of a two-port network.

In the pulse generator case, the ability of the measurement system to provide a waveform that is a near replica of the pulse depends upon the static calibration factors and the dynamic response or shape of the measurement system's step-response transition. Consequently, the 10\%-to- $90 \%$ transition duration of the waveform will always be in error in relation to that of the step-like pulse being observed by the measurement system.

For the two-port network case, errors due to noise, measurement system instability, and static-calibration-factor errors prevent the precise determination of the insertion impulse response. The integral of the resultant impulse response waveform yields the step response waveform whose $10 \%$-to- $90 \%$ transition duration will always be in error as compared to that of the unobservable step response of the two-port network.

The linear, time-invariant, dynamic effects that prevent the exact measurement of transition duration are due to convolutional processes. The partial removal or reduction of such effects is called deconvolution. The deconvolution process for the signal generator case is different from that of the two-port network case. In the remaining part of this Section, the following topics are discussed: (3.4.2) Deconvolution, (3.4.3) Typical Results for Pulse Generator Measurements, and (3.4.4) Typical Results for Two-Port Network Measurements.

\subsubsection{Deconvolution}

Deconvolution is the process of removing the effects of a convolution operation. Physically, when a given signal, $x(t)$, excites a causal, linear, time-invariant system whose impulse response is $h(t)$, the output of the linear system, $y(t)$, is given by the convolution equation or integral, Fig 3.7A [15].

$$
y(t)=\int_{0}^{t} x(\lambda) h(t-\lambda) d \lambda=\int_{0}^{t} x(t-\lambda) h(\lambda) d \lambda .
$$

As indicated, the integral may be written in either form. $x(t)$ is said to be convolved with $h(t)$. For the purposes of this monograph, in the linear systems considered here, the deterministic function, $h(t)$, will be stationary, that is, the deterministic-system properties do not change with time. A compact notational form for eq (3.1) is written as,

$$
y(t)=x(t) \star h(t),
$$


where the star denotes the convolution operation.

Inspection of the convolution integral in Eq. (3.1) shows that the roles of $x(t)$ and $h(t)$ are interchangeable, that is, it also can be said that $h(t)$ is convolved with $x(t)$. The important point here is that $x(t)$ and $h(t)$ are the two known functions and the integrals in Eq. (3.1) yield the unknown function, $y(t)$.

If $y(t)$ is a known function while only one of the integrand functions is known, then an integral equation results whose solution would yield the unknown integrand function. The solution of the integral equation is called deconvolution. If $y(t)$ and $h(t)$ are known functions, then $x(t)$ is said to be deconvolved from $y(t)$ and $h(t)$; similarly, for $y(t)$ and $x(t)$ known, $h(t)$ is said to be deconvolved from $y(t)$ and $x(t)$. These two cases are illustrated by the block diagrams in Figs 3.7B and 3.7C.

When the input signal $x(t)$ is unknown, Fig 3.7B, the integral equation is called the signal identification problem, or in the pulse calibration case, the pulse generator problem.

$$
y(t)=\int_{0}^{t} x(\lambda) h(t-\lambda) d \lambda, \quad x(t) \text { unknown. }
$$

On the other hand, if the system impulse response $h(t)$ is unknown, the integral equation is called the system identification problem, or in the two-port network case, the low-pass filter problem.

$$
y(t)=\int_{0}^{t} x(\lambda) h(t-\lambda) d \lambda, \quad h(t) \text { unknown. }
$$

In an actual physical measurement situation the signals or data defined as $x(t), y(t)$, and $h(t)$ are most likely to be in error to some degree due to noise, interference, equipment drifts, inadequate modeling, etc. Let $x_{0}(t), y_{0}(t)$, and $h_{0}(t)$ to be the true values and assume that the errors for these functions can be represented as the additive terms, $n_{x}(t)$, $n_{y}(t)$, and $n_{h}(t)$, respectively, Fig 3.8. Consequently $x(t), y(t)$, and $h(t)$ are written as,

$$
x(t)=x_{0}(t)+n_{x}(t)
$$




$$
y(t)=y_{0}(t)+n_{y}(t)
$$

and,

$$
h(t)=h_{0}(t)+n_{h}(t) .
$$

To avoid any confusion in the use of the term error, these departures from the conceptual true values will simply be called noise components, or noise. The presence of these noise components prevents the exact recovery of $x_{0}(t)$ and $h_{0}(t)$ by deconvolution in the pulse generator problem and in the two-port network problem, respectively. Furthermore, the presence of noise usually introduces (irregular) instability into the deconvolution process which in turn requires that some action be taken to achieve a stable (regular) result. ${ }^{4}$

Briefly, the cause of the instability can be qualitatively recognized by substituting the relevant equations from Eqs. (3.5) through (3.7) into the convolution integral for each problem [Eq. (3.3) or Eq. (3.4)]. For this qualitative discussion assume that the noise components are causal $\left(n_{\mathrm{i}}=0, t<0\right)$, then for the two problems, the following equations are obtained.

$$
\begin{aligned}
& \text { Pulse Generator: } y_{0}(t)+n_{y}(t) \neq \int_{0}^{t} x_{0}(\lambda)\left[h_{0}(t-\lambda)+n_{h}(t-\lambda)\right] d \lambda . \\
& \text { Two-Port Network: } y_{0}(t)+n_{y}(t) \neq \int_{0}^{t}\left[x_{0}(\lambda)+n_{x}(\lambda)\right] h_{0}(t-\lambda) d \lambda .
\end{aligned}
$$

The inequality results from the facts that,

${ }^{4}$ Actually, the irregular result is repeatable with a given set of data and is not due to instability in computation. Typically the irregular result does not converge to a regular smooth curve having a general shape conforming to the mind's eye picture held by the operator performing the deconvolution. Commonly, in deconvolution problems the terms stable (unstable) and regular (irregular) have the same meaning and are interchangeable. 


$$
\begin{aligned}
& \text { Pulse Generator: } n_{y}(t) \neq \int_{0}^{t} x_{0}(\lambda) n_{h}(t-\lambda) d \lambda . \\
& \text { Two-Port Network: } n_{y}(t) \neq \int_{0}^{t} n_{x}(\lambda) n_{0}(t-\lambda) d \lambda .
\end{aligned}
$$

even though for both problems,

$$
y_{0}(t)=\int_{0}^{t} x_{0}(\lambda) h_{0}(t-\lambda) d \lambda .
$$

$n_{y}(t)$ is not related to $n_{h}(t)$ through the convolution of the latter with $x_{0}(t)$, nor is $n_{y}(t)$ similarly related to $n_{x}(t)$ and $h_{0}(t)$. The situation is due to the fact that the noise components include some unrelated phenomena. For example, $n_{y}(t)$ may include an instrumentation drift, while $n_{h}(t)$ may be due to modeling errors, and $n_{x}(t)$ may include some interference signal. These noise components are the residual components which remain even after averaging or other signal processing of data ensembles.

It is shown in [28] that by using filtering the deconvolution process can be stabilized and that the convolution process cannot be exactly reversed by deconvolution. This means that the result is always an estimate or approximation to the desired result which yields for the two problems,

$$
\begin{aligned}
& \text { Pulse Generator: } d_{x}(t)=\int_{0}^{t} x_{0}(\lambda) f_{x}(t-\lambda) d \lambda . \\
& \text { Two-Port Network: } d_{h}(t)=\int_{0}^{t} h_{0}(\lambda) f_{h}(t-\lambda) d \lambda .
\end{aligned}
$$

where the $d_{i}(t)$ denotes a deconvolution result which approximates $x_{0}(t)$ or $h_{0}(t)$ as the case may be. If $f_{i}(t)$ were a Dirac delta function, $(\delta(t))$, then $d_{i}(t)$ would reduce to $x_{0}(t)$ or $h_{0}(t)$. 
The function $f_{i}(t)$ is the impulse response of the overall filtering function. In the frequency domain $F_{i}(j \omega)$ is the product of two functions,

$$
F_{i}(j \omega)=\mathscr{F}\left[f_{i}(t)\right]=K_{i}(j \omega) R_{i}(Y, j \omega)
$$

where $\mathscr{F}$ denotes the Fourier transformation operator. $K_{\mathrm{i}}(j \omega)$ is the noise term that produces the instability in the deconvolution, and $R_{\mathrm{i}}(j \omega)$ is the regularization filter that suppresses the instability or irregularity introduced by $K_{\mathrm{i}}(j \omega)$. The strength of the filtering depends upon $\gamma, 0 \leq \gamma \leq \infty$. If it were possible to make

$$
K_{i}(j \omega) R_{i}(Y, j \omega)=1
$$

then

$$
f_{i}(t)=\delta(t)
$$

and

$$
d_{i}(t)=\left\{\begin{array}{ll}
x_{0}(t), & i=x \\
h_{0}(t), & i=h
\end{array}\right\}
$$

which is perfect deconvolution. Since perfect filtering is not achievable, the least that can be expected is to achieve a stable result while the most is to achieve a stable result that approximates our mind's eye concept of the proper result. Thus, it becomes clear that a subjective decision must be made by the individual performing the deconvolution as to when the filtering is adequate to provide a good enough approximation to his or her mind's eye picture of the proper result, $x_{0}(t)$ or $h_{0}(t)$. To emphasize this point, it should be recalled that $x_{0}(t)$ and $h_{0}(t)$ are generally inaccessible and therefore generally unknown; consequently, the operator should have a conception of the form of a proper result.

In both the pulse generator problem and the two-port network problem, a subjective judgement must be made and making that judgement is difficult. Within limits, the decision procedure can be automated using a computer and will depend upon the deconvolution method employed. The deconvolution method used by NBS is based upon optimization theory and is implemented in the frequency domain via Fourier transforms with the deconvolution results being inversely transformed back into the time domain $[16,28]$. The process is automated using an iterative process controlled by decision criteria implemented in the time domain. The complete automated deconvolution process has also been applied to the measurement of the impulse response of optical fibers by Guillaume and Bizeul [17]; however, a theoretical basis for the automated method was not developed 
at that time and is given in [28]. Somewhat later (1982-3), Parruck and Riad developed a different filter optimization technique which greatly reduced the operator's influence on the result [38].

As indicated in Eq. (3.15) the regularization filter $R_{\mathrm{i}}(\gamma, j \omega)$ is a function of the positive real parameter $Y$, which controls the amount or strength of the filtering. For the two problems, $R_{\mathrm{i}}(Y, j \omega)$ is given by,

$$
\begin{aligned}
& \text { Pulse Generator: } R_{x}(Y, j \omega)=\frac{|H(j \omega)|^{2}}{|H(j \omega)|^{2}+Y_{X}|C(j \omega)|^{2}} . \\
& \text { Two-Port Network: } R_{h}(Y, j \omega)=\frac{|X(j \omega)|^{2}}{|X(j \omega)|^{2}+Y_{h}|C(j \omega)|^{2}} .
\end{aligned}
$$

where,

$$
C(j \omega)=(j \omega)^{2} .
$$

The function $C(j \omega)$ is the Fourier transform of the second derivative operator $d^{2} / d t^{2}$; it appears in the filter due to the optimization constraint which requires that the deconvolution result be smooth (minimize the second derivative of the result). $V_{i}$ is adjusted by the automation procedure to the optimal value $Y_{i_{0}}$ as determined by the automation constraints.

Summarizing, the deconvolution process does not exactly reverse the convolution process and provide $x_{0}(t)$ for the pulse generator signal or $h_{0}(t)$ for the two-port network impulse response. Instead, it provides an approximation for each desired result, in particular,

$$
\text { Pulse Generator: } d_{x}(t)=\int_{0}^{t} x_{0}(\lambda) f_{x}\left(t-\lambda, Y_{x}\right) d \lambda
$$




$$
\text { Two-Port Network: } d_{h}(t)=\int_{0}^{t} h_{0}(\lambda) f_{h}\left(t-\lambda, V_{h}\right) d \lambda
$$

where Eqs. (3.13) and (3.14) have been written to show explicitly their dependence upon $\gamma$. The optimal value of $\gamma, \gamma_{0}$, decided upon in a given case is determined through a subjective judgement made by the individual manually performing the deconvolution, or made by the computer automatically performing the deconvolution. The optimal deconvolution results are then $\left[d_{x}(t)\right]_{y \times 0}$ and $\left[d_{h}(t)\right]_{y h o}$, respectively.

$$
\begin{aligned}
& \text { Pulse Generator: }\left[d_{x}(t)\right]_{y \times 0}=\int_{0}^{t} x_{0}(\lambda) f_{x}\left(t-\lambda, Y_{x 0}\right) d \lambda . \\
& \text { Two-Port Network: }\left[d_{h}(t)\right]_{Y h 0}=\int_{0}^{t} h_{0}(\lambda) f_{h}\left(t-\lambda, Y_{h 0}\right) d \lambda .
\end{aligned}
$$

(With respect to notation, here the optimum values of $y$ are designated with an $x$ or $h$ subscript to avoid giving the impression that $\gamma_{x_{0}}$ and $\gamma_{h_{0}}$ are the same quantity. In the remainder of this document the subscripts $x$ and $h$ may not be appended to $y$ if no possibility for confusion exists.)

\subsubsection{Pulse Generator Measurements}

A pulse generator measurement was implemented using the system shown in Fig 3.9. The blocks $\mathrm{C}-\mathrm{C}$ represent a connector-pair, with one of the pair being part of the pulse generator $x(n \Delta t)$ while the other belongs to the measurement system $h(n \Delta t)$.

Because the waveforms are sequences (sampled-data) instead of continuous data, the time-domain dependent variables, $x, h$, and $y$, are written with the independent variable, $t$, expressed as $(n \Delta t)$, where $n$ is an integer denoting the sample number and $\Delta t$ is a constant representing the sampling-time interval. For brevity, $n \Delta t$ is written as $n$ with $\Delta t$ understood.

In the pulse generator problem, $y(n)$ and $h(n)$ are given or known with $y(n)$ being obtained from averaged experimental data and $h(n)$ from a physically based mathematical model. In the stated notation, they are written, 


$$
y(n)=y(0), y(1), y(2), \ldots, y(N-1) ; 0<n<N-1
$$

and,

$$
h(n)=h(0), h(1), h(2), \ldots, h(N-1) ; 0<n<N-1 \text {. }
$$

where the total duration of each sequence is $N \Delta t$, that is, the total number of equally spaced samples is $N$. In principle (exactly for noise free data), $y(n)$ is equal to the convolution of $x(n)$ with $h(n)$,

$$
y(n)=x(n) \star h(n),
$$

or inversely, the unknown pulse generator waveform $x(n)$ is equal to the deconvolution of $y(n)$ with $h(n)$,

$$
x(n)=y(n) \circlearrowleft h(n),
$$

where denotes deconvolution, and where,

$$
x(n)=x(0), x(1), x(2), \ldots, x(N-1) ; 0<n<N-1 .
$$

The deconvolution is implemented in the discrete frequency domain by first transforming $y(n)$ and $h(n)$ into the frequency domain by means of the discrete Fourier transform using a fast Fourier transform (FFT) algorithm $[18,19]$. The discrete frequency-domain sequences are written,

$$
Y(k)=Y(0), Y(1), Y(2), \ldots, Y(N-1) ; 0<k<N-1,
$$

and,

$$
H(k)=H(0), H(1), H(2), \ldots, H(N-1) ; 0<k<N-1 \text {, }
$$

where in general each value is complex. The deconvolution is then performed by division yielding the sequence,

$$
X(k)=\frac{Y(k)}{H(k)},
$$


where,

$$
X(k)=X(0), X(1), X(2), \ldots, X(N-1) ; 0<k<N-1 .
$$

Direct inversion back to the time domain by means of the inverse FFT of the $X(k)$ sequence generally yields an unstable result. As discussed in Section 3.4.2, the Fourier transform must be multiplied by a regularizing filter $\mathrm{R}\left(k, Y_{0}\right)$ before inverting back to the time (sequence) domain.

$$
\left[D_{x}(k)\right]_{y_{0}}=X(k) R_{x}\left(k, y_{0}\right)
$$

where,

$$
R_{x}\left(k, Y_{0}\right)=\frac{|H(k)|^{2}}{|H(k)|^{2}+Y_{x_{0}}|C(k)|^{2}},
$$

and where the squared magnitude of the FFT of the second difference operator may be written as [16],

$$
|C(k)|^{2}=16 \sin ^{4}\left(\frac{\pi k}{N}\right) ; \quad 0<k<N-1 .
$$

In Eqs. (3.31) through (3.37), for brevity, the argument of the sequences has been written as $k$ rather than the actual value $k \Delta \Omega$, where,

$$
\Delta \Omega=\frac{1}{N \Delta t} \text {. }
$$

The inverse FFT of (3.35) yields a time sequence that is the desired estimated sequence for $x_{0}(n)$, which may be expressed as,

$$
\left[d_{x}(n)\right]_{Y_{0}}=\left[d_{x}(0), d_{x}(1), d_{x}(2), \ldots, d_{x}(N-1)\right]_{Y_{0}} ; 0<n<N-1 .
$$

Fig 3.10 shows a typical set of sampled-data waveforms obtained for a pulse generator measurement. The sequences consisted of 512 sample values uniformly spread over a $1 \mathrm{~ns}$ time window. The pulse generator was an NBS reference waveform standard having a nominal transition duration of $100 \mathrm{ps}$. The observed transition duration was $103.6 \mathrm{ps}$ as obtained by the NBS APMS using a point-by-point average of 300 waveforms, Fig 3.10A. 
The measurement system dynamic characteristics were represented by the sampling-head model discussed in Section 3.2.2, which possessed a step-response transition duration of 20.85 ps, Fig 3.10B. The estimated pulse generator waveform had a transition duration of $97.4 \mathrm{ps}$ and is shown in Fig 3.10C.

\subsubsection{Typical Results for Low Pass Filter Measurements}

A low-pass filter measurement was implemented using the system shown in Fig 3.11. The measurement method is that of insertion, which provides two waveforms corresponding to the situation before and after insertion of the filter. Viewing the process in terms of the frequency-domain transforms for the two waveforms, the ratio of the insertion waveform transform to that of the reference waveform is equal to the scattering transfer function, $S_{21}(j \omega)$. The inverse Fourier transform of $S_{21}(j \omega)$ is defined as the insertion impulse response of the inserted element, $h(t)$. The insertion step-response of the low-pass filter is equal to the integral of $h(t)$ over the limits of zero to $t$.

In this case, the known waveforms are the ensemble-averaged waveform sequences,

$$
x(n)=x(0), x(1), x(2), \ldots, x(N-1) ; 0<n<N-1,
$$

and,

$$
y(n)=y(0), y(1), y(2), \ldots, y(N-1) ; 0<n<N-1 .
$$

Here, $x(n)$ and $y(n)$ are the sequences or discrete waveforms representing the sampled data of the reference (before insertion) waveform and the response (after insertion) waveform, respectively.

Proceeding in the same manner as in the pulse generator problem (Section 3.4.3), the discrete frequency-domain insertion transfer function $H(k)$ is given by,

$$
H(k)=\frac{Y(k)}{X(k)} \text {. }
$$

The transfer function corresponding to the regularized estimate is,

$$
\left[D_{h}(k)\right]_{\mathrm{y}_{0}}=H(k) R_{h}\left(k, \mathrm{Y}_{0}\right) \text {, }
$$


The transfer function corresponding to the regularized estimate is,

$$
\left[D_{h}(k)\right]_{\mathrm{y}_{0}}=H(k) R_{h}\left(k, \mathrm{y}_{0}\right) \text {, }
$$

where,

$$
R_{h}\left(k, Y_{0}\right)=\frac{|X(k)|^{2}}{|X(k)|^{2}+Y_{h_{0}}|C(k)|^{2}} .
$$

Consequently, the inverse FFT of $\left[D_{h}(k)\right]_{\mathrm{ro}}$ in Eq. (3.43) gives the time-domain sequence for the estimate of the impulse response, $h_{0}(k)$.

$$
\left[d_{h}(n)\right]_{Y_{0}}=\left[d_{h}(0), d_{h}(1), d_{h}(2), \ldots, d_{h}(N-1)\right]_{Y_{0}} ; 0<n<N-1 .
$$

The step-response sequence is equal to the discrete-integral of the discrete impulse response,

$$
\left[d_{u}(n)\right]_{\mathrm{Y}_{0}}=\sum_{i=0}^{n}\left[d_{h}(i)\right]_{\mathrm{Y}_{0}} ; \quad 0<n<N-1
$$

Figs 3.12 and 3.13 show a typical set of acquired and deconvolved waveforms as encountered in a low-pass filter measurement. Fig 3.12 shows the acquired waveforms for an insertion measurement of a $3.5 \mathrm{GHz}$ commercial low-pass filter; here the insertion waveforms $x(n)$ and $y(n)$ are each the result of an ensemble average of 100 waveforms. The deconvolution estimate for the impulse response is shown in Fig 3.13A while its integral, the estimated step response, is shown in Fig 3.13B. The estimated step-response transition duration is $124.8 \mathrm{ps}$.

\subsection{Uncertainty Analysis}

\subsubsection{General Sources of Error}

There are three general sources of error which arise from relatively separate origins; they are:

1. Sampling-head model error

2. System calibration error

3. Deconvolution error. 
sampling head. Because the deconvolution procedure is necessarily an estimation process, the procedure decreases the accuracy.

In the next three sections, the methods used in the reporting period for calculating the errors from the three sources listed above will be described.

\subsubsection{Sampling-Head Error}

At the time of this work, the step-response transition duration error bounds of the sampling-head were specified along with its nominal transition duration value. These were $20(+3,-5)$ ps and were mainly established from NBS calibration-service data developed in the calibration of tunnel diode step-like generators [20]. The nominal value of the sampling-head transition duration was 20 ps and was equal to the transition duration for the step-response of the sampler model.

\subsubsection{System Calibration Error}

Amplitude [21] and time base [22] standards were used to calibrate the vertical and horizontal deflection channels, respectively.

The amplitude calibrator used had the following specifications and provisions:

1. The amplitude voltage accuracy was within $\pm 0.25 \%$ (typically $<0.1 \%$ ) except $\pm 0.4 \%$ at $2 \mathrm{mV}$ and $\pm 0.7 \%$ at $1 \mathrm{mV}$ for a range of $\pm(1 \mathrm{mV}$ to $5 \mathrm{~V})$.

2. Three selectable output impedances were available: (a) $<0.1 \Omega$, (b) $(50 \pm 0.05) \Omega$, and (c) $(1 \pm 0.001) \mathrm{M} \Omega$.

3. The operating modes were $\mathrm{DC}$ or a $(1 \pm 0.0001) \mathrm{kHz}$ square-wave having a baseline of zero volts.

4. Selectable sampling-head protection circuitry limited the maximum output voltage to $\pm 1.8 \mathrm{~V}$ nominal.

The amplitude calibrator was set to ascending, equally spaced voltage values such that the minimum and maximum calibrator voltages bound the voltage values of the waveform to be measured. For each calibrator voltage, a number of $\mathrm{N}$-point sweeps were acquired by the APMS and the mean value of all of the sample values was computed. The APMS then stored each of the calibrator nominal values and their associated APMS-indicated values into a disk file correction table. This file was subsequently used to correct the values of the recorded pulse waveform in order to remove gain and nonlinearity errors.

The time base standard was a pulsed wavemeter with a relative expanded uncertainty of $\pm 0.2 \%$. Similar to the voltage calibration case, the APMS recorded the pulsed sine 
wave from the standard and computed the sampling oscilloscope's indicated times for the equi-slope, zero crossings of the signal. These values were stored into another disk-file correction table and later used to correct the recorded pulse waveform for time base gain and nonlinearity errors.

\subsubsection{Deconvolution Error - Pulse Generator Problem}

The deconvolution process attempts to remove the dynamic effects of the measurement system, that is, those effects that produce a waveform whose variations are slower than those of the pulse being measured.

For the pulse generator problem there are two error components involved in the error calculations, those due to (1) the sampling-head model and (2) those due to the deconvolution estimate itself. For example, Fig 3.9, given the measured waveform, $y(n \Delta t)$, corresponding to the pulse being measured, $x(n \Delta t)$, and the measurement system impulse response (model), $h(n \Delta t)$, the corresponding FFT's yield,

$$
X(k \Delta \Omega)=\frac{Y(k \Delta \Omega)}{H(k \Delta \Omega)} .
$$

Here, in principle, division by $H(k)$ removes the effects of the non-zero transition duration of the measurement system. However, if $H(k)$ itself is in error, then $X(k)$ will be in error.

Because the deconvolution procedure requires filtering with an optimal filter, for example, that shown in Eq. (3.36), the filtering operation introduces an error. This can be understood by referring to Fig 3.14 in which the estimate for the frequency domain spectrum magnitude of the pulse generator is given. Comparing the filtered and unfiltered estimates, it is seen that the filtered spectrum apparently follows the unfiltered spectrum down to about $-40 \mathrm{~dB}$. Actually, there is a slight increase above the attenuation of the unfiltered spectrum. The true spectrum is contained within the unfiltered spectrum, but it only can be seen when the irregular component (that beyond about $12.5 \mathrm{GHz}$ ) is made negligible and not allowed to dominate the spectrum above $12.5 \mathrm{GHz}$. Recall that the causes of the irregularity are contained in Eq. (3.33). Therefore, the true spectrum for all $k$ is not available and the error due to filtering cannot be based upon it.

If the complex unfiltered spectrum estimate were to be inverse-Fourier-transformed (inverse FFT) to yield a time-domain waveform, the result would be irregular and would bear little resemblance to the true generator waveform. The filtering operation is necessary to prevent the irregular component from overriding the true component. 
To obtain a measure of the filtering effects, a first order perturbation will be applied to the optimal filtering results in order to estimate the transition duration without the (necessary) application of the filter. In other words, this estimate will yield a slightly greater $3 \mathrm{~dB}$ frequency, which will correspond to a slightly smaller transition duration for the pulse generator output. Because the perturbation will be small, the nonlinear property of the filter will be disregarded, and the problem will be treated as a linear one. The nonlinearity of the filter is due to its dependence on the impulse response, $H(k)$, Eq. (3.36).

Proceeding, using the tabulated data corresponding to the pulse measurements and optimal filtering results shown in Figs 3.10 and 3.14B, the optimal transition duration and $3 \mathrm{~dB}$ frequency are $97.4195 \mathrm{ps}$ and $3.5121 \mathrm{GHz}$, respectively. Denote these quantities as $\mathrm{TD}_{0}$ and $k_{0} \Delta \Omega$ and form their product,

$$
K=T D_{0} k_{0} \Delta \Omega
$$

where $\Delta \Omega$ is the discrete frequency spacing. $K$ is a constant for all systems in the class of systems that have the response-shapes in the neighborhood of $D\left(k, Y_{0}\right)$ and $d\left(n, Y_{0}\right)$. Now because the $3 \mathrm{~dB}$ frequency of the filtered result is only slightly different than that of the unfiltered result, define an equivalent true response in the same class as $d\left(n, Y_{0}\right)$,

$$
K=T D_{R} k_{R} \Delta \Omega \text {. }
$$

Two ways of characterizing the error in the measurement system (sampling head) for the pulse generator problem are as follows:

1. Step response transition duration bounds in terms of a principal value with lower and upper limits, e.g., $30(+4,-6)$ ps.

2. An impulse response waveform.

In the first way, the deconvolution estimate is a simple error-free numerical calculation. The latter method requires computer calculations employing a deconvolution algorithm; the algorithm itself introduces an error that may be removed from the final result for the transition duration.

If only sampling-head transition-duration bounds are given, then the deconvolution process necessarily relies upon the root of the sum of the squares of the transition durations involved $[29,30]$, that is, 


$$
T D M=\left[T D R^{2}+T D S^{2}\right]^{1 / 2},
$$

where TDM, TDR, and TDS are the measured transition duration, the true transition duration for the step-response being measured, and the sampling-head transition duration, respectively. For the sampling-head transition duration as specified in Section 3.5.2 and the typical results for a measured transition duration, Section 3.5.3, the true transition duration would be,

$$
T D R=\left[(32.475 p s)^{2}-\left(20_{-5}^{+3}\right)^{2}\right]^{1 / 2},
$$

or,

$$
T D R=25.586_{-3.204}^{+3.647} \mathrm{ps} .
$$

Since, the ratio of Eq. (3.48) to Eq. (3.49) gives,

$$
\frac{T D_{0} k_{0} \Delta \Omega}{T D_{R} k_{R} \Delta \Omega}=1
$$

then,

$$
T D_{R}=T D_{0} \frac{k_{0} \Delta \Omega}{k_{R} \Delta \Omega} .
$$

For example, if $k_{0} \Delta \Omega$ is one-percent less than $k_{R} \Delta \Omega$, the true-response transition duration would be one-percent less than that for the optimum-filtered result. This relationship provides a means for setting bounds on the effect of the deconvolution operation. Using the values cited above, $K=0.3422$. The tabulated data for the unfiltered result, Fig 3.14A, gives a $3 \mathrm{~dB}$ frequency of $3.5124 \mathrm{GHz}$. Putting these values into Eq. (3.54) gives $T D_{R}=97.3978$ ps which is 0.0172 ps less than the optimal result. Thus, the deconvolution routine has little influence on the result. 


\subsubsection{Deconvolution Error - Low Pass Filter Problem}

In the low-pass filter problem, the measurement system consists of the cascade connection of a given pulse generator and a sampling oscilloscope, Fig 3.11. The measurement system is usually a $50 \Omega$ system in which the time windows are chosen so that $50 \Omega$ sources and loads are maintained for the pulse signals being observed; the measurement method is a time-domain insertion method [14]. The result of the measurement is an estimate for the insertion impulse response, that is, the Fourier transform of the insertion or scattering parameter $S_{21}(j \omega)$.

For the low pass filter problem discussed in Section 3.4.4, the magnitude of the deconvolution estimate, $D_{h}(k)$, is shown in Fig 3.15. The time-domain optimal estimate, $d_{n}\left(n, V_{0}\right)$, corresponding to Fig 3.15B is shown in Fig 3.13. Using tabulated values for $\left|D_{h}(k, \gamma)\right|$ it is found that the $3 \mathrm{~dB}$ attenuation frequencies are $3.585 \mathrm{GHz}$ and $3.592 \mathrm{GHz}$ for the optimal filter and the unfiltered result, respectively. Multiplying the $10 \%$-to- $90 \%$ transition duration of the optimally filtered result, $124.8 \mathrm{ps}$, by $3.585 \mathrm{GHz}$ yields the constant $K=0.447$, Eq. (3.48). Dividing $K$ by $3.592 \mathrm{GHz}$ gives the estimate for $T D_{R}=$ 124.4 ps. Thus the deconvolution operation has a negligible effect on the transition duration estimate. 


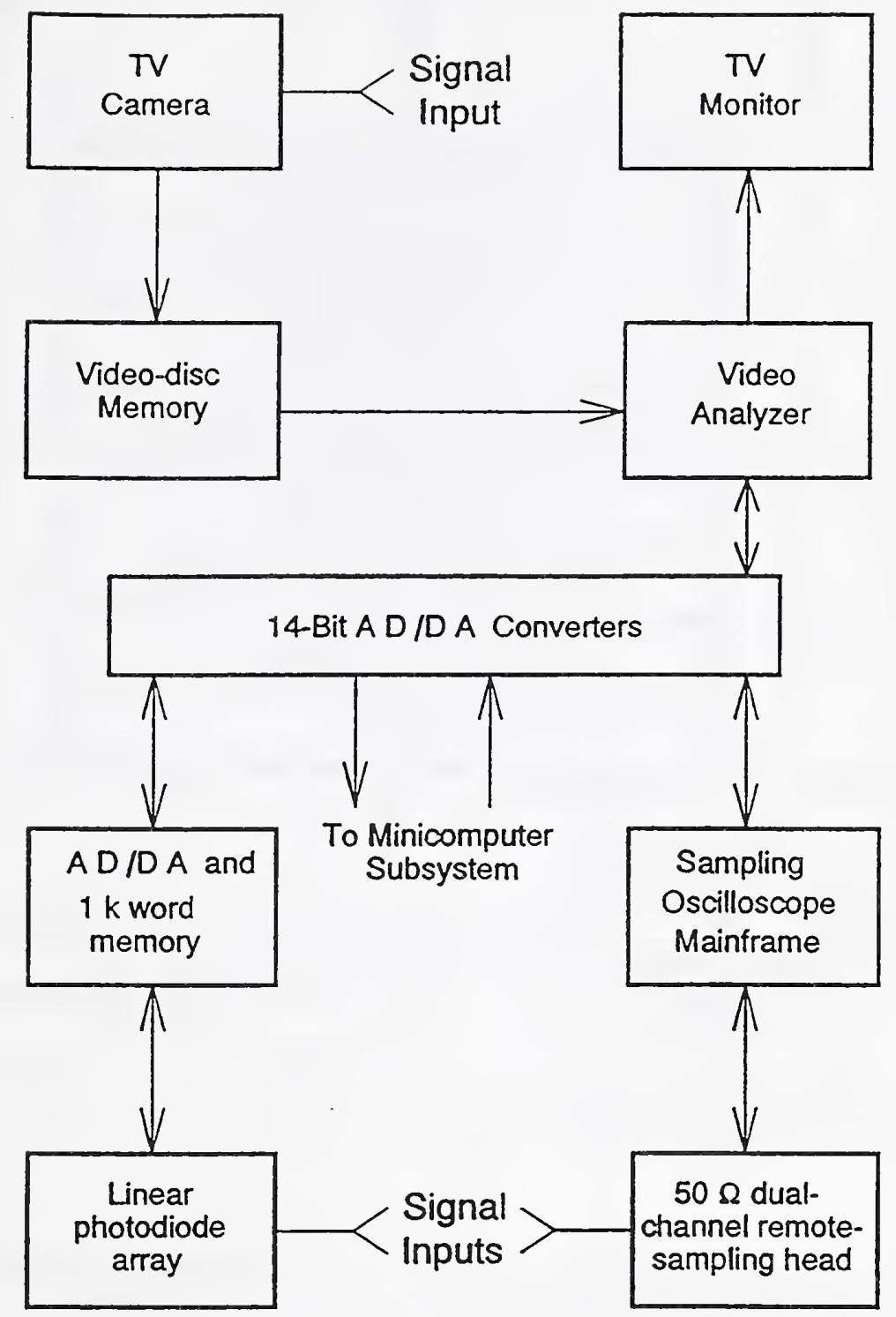

Figure 3.1 The Automatic Pulse Measurement System (APMS). 


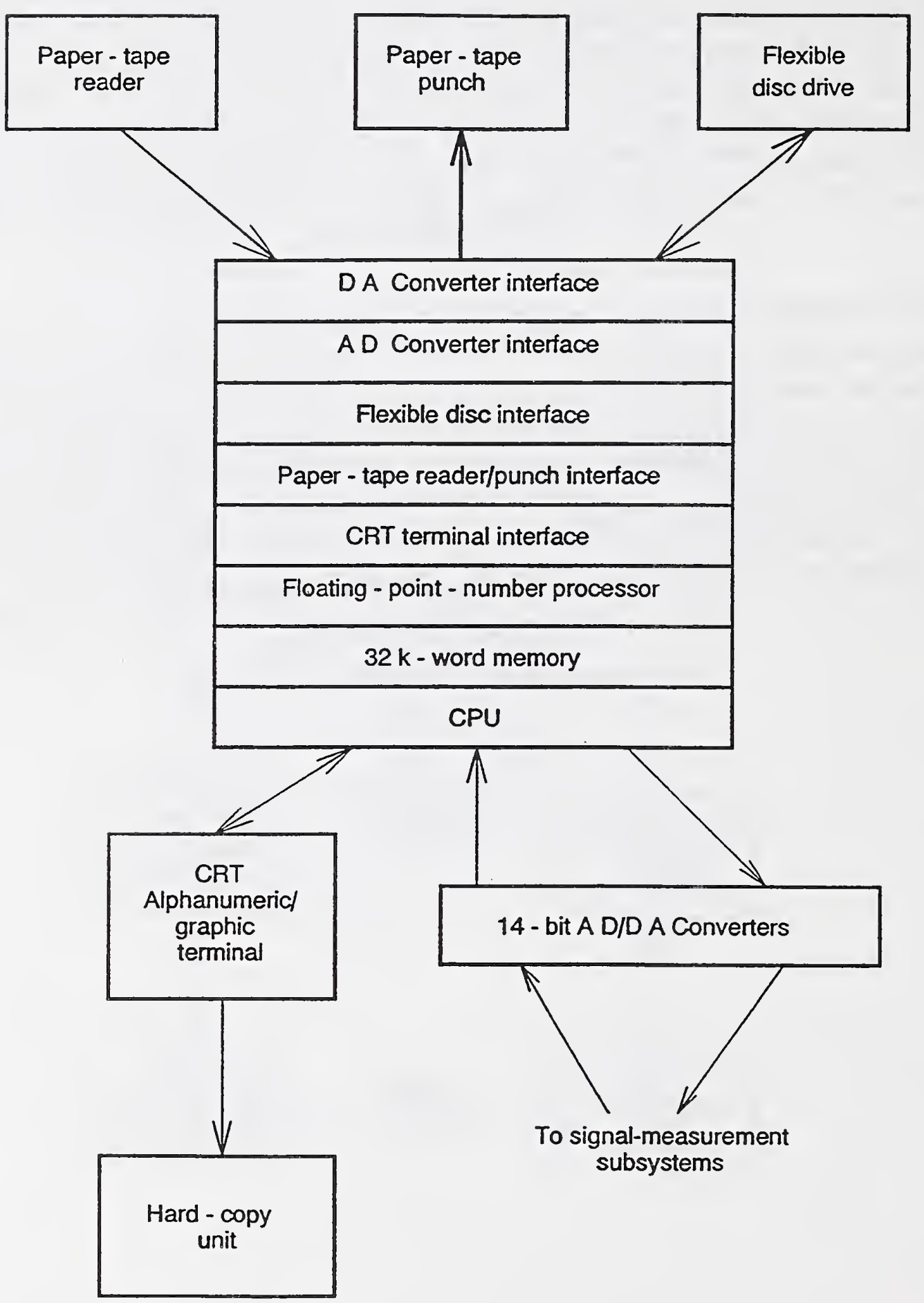

Figure 3.2 The APMS minicomputer subsystem. 


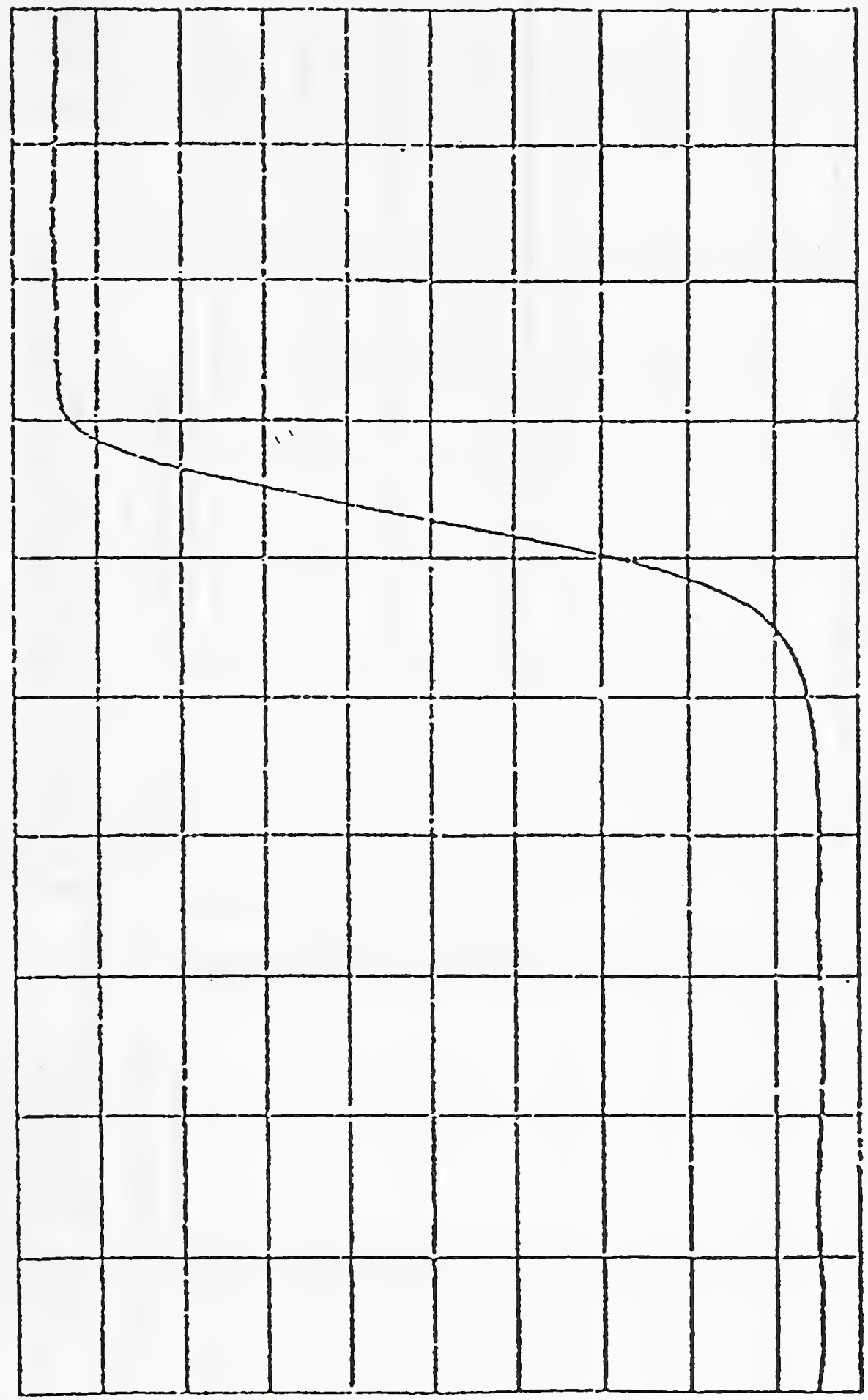

Figure 3.3 HP-1430A 28 ps sampling head step response; vertical scale factor is $111.1 \mathrm{mV} / \mathrm{div}$ and time scale factor is $25.6 \mathrm{ps} / \mathrm{div}$; response bias $=-1.76 \mathrm{~V}$. 


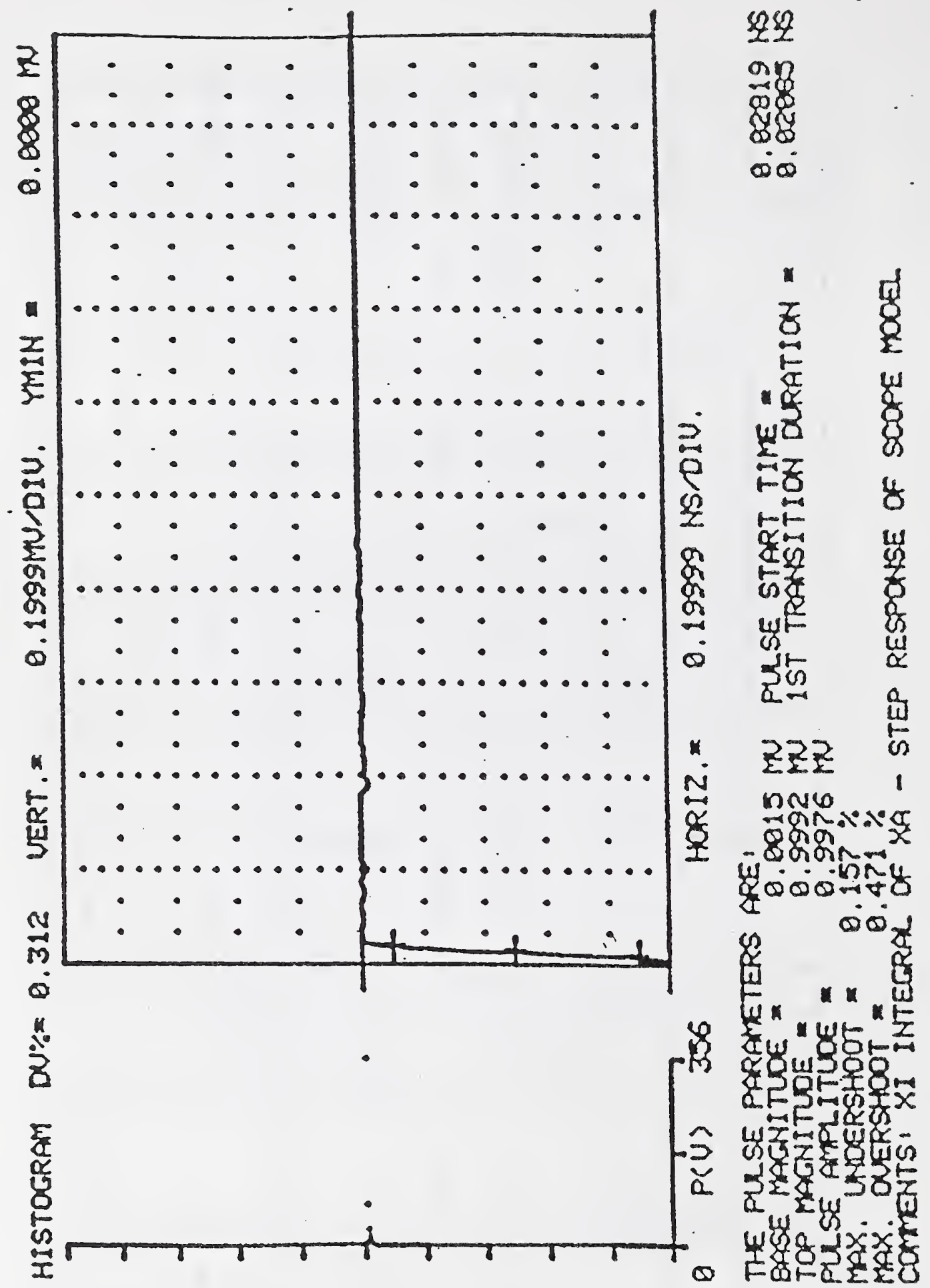

Figure 3.4 The step response and probability density histogram of the model for the Hewlett-Packard Model 1430B sampling head used in the NBS APMS. 


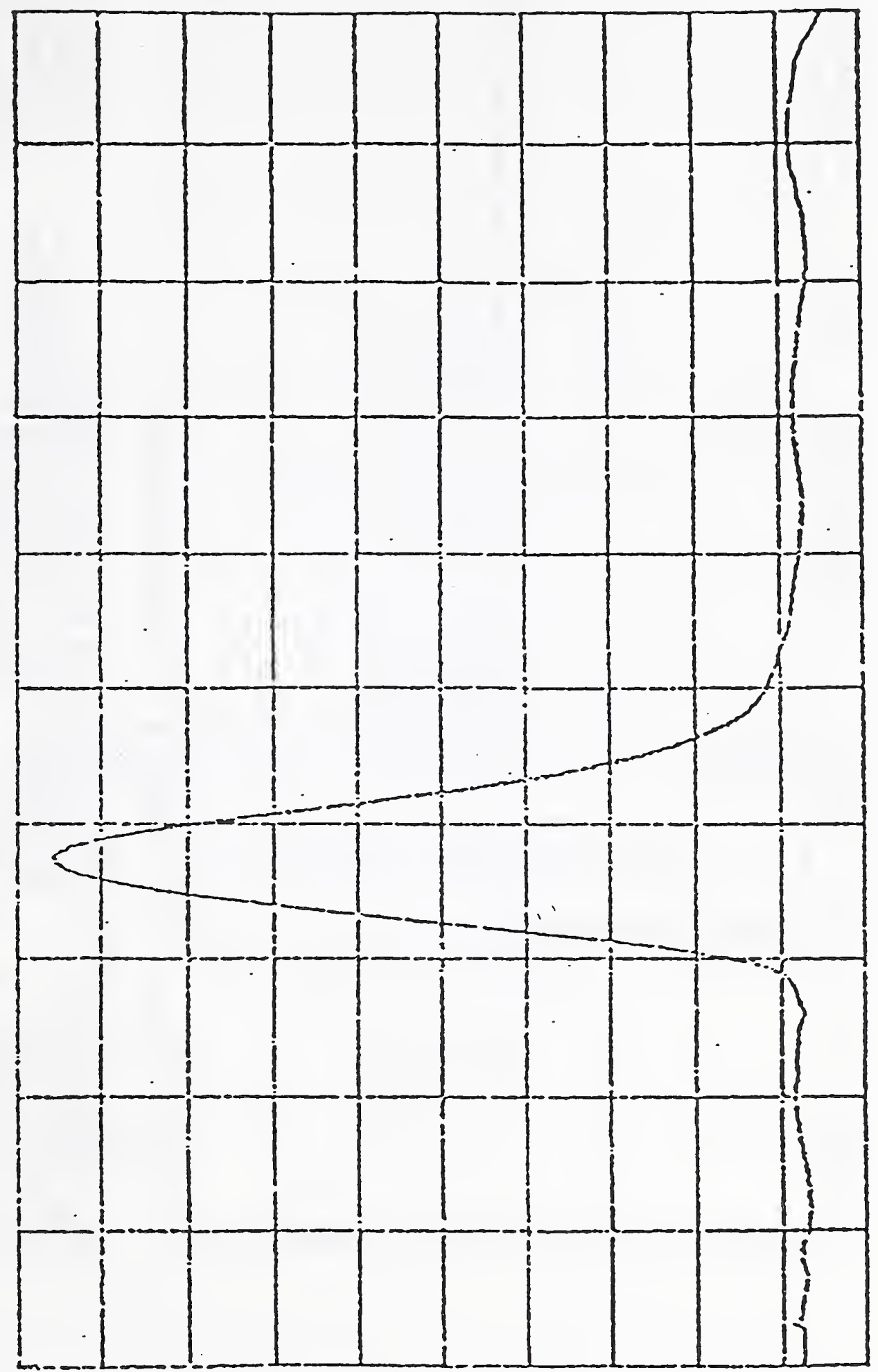

Figure 3.5 HP-1430A 28 ps sampling head impulse response; vertical scale factor is arbitrary and time scale factor is $25.6 \mathrm{ps} / \mathrm{div}$; response bias $=-1.76 \mathrm{~V}$. 


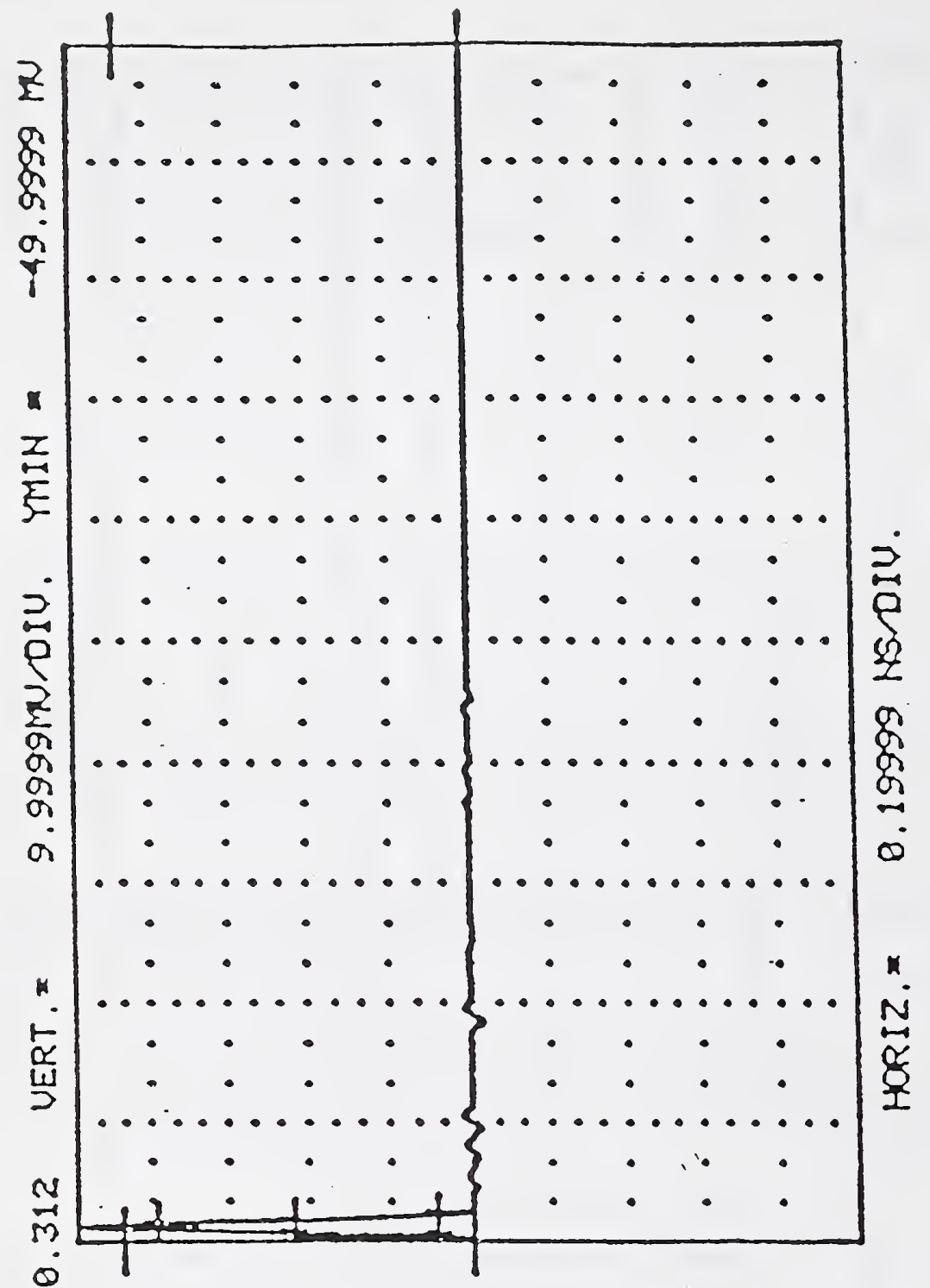

4)

nom $2^{2}$

पर्पर

$080 \rightarrow 0$

$000-13$

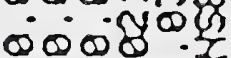

$\infty^{-\infty}$ है

(1)

₹z जै

네

䍐察

- ç

जठㅁㅁ

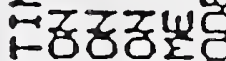

manten t5t-5

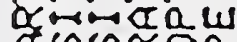

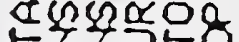
csacos

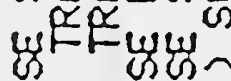

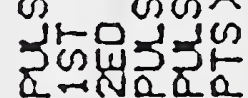

- र2z

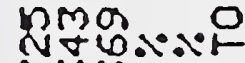
Nom

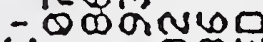

से Qgman

\& लmo
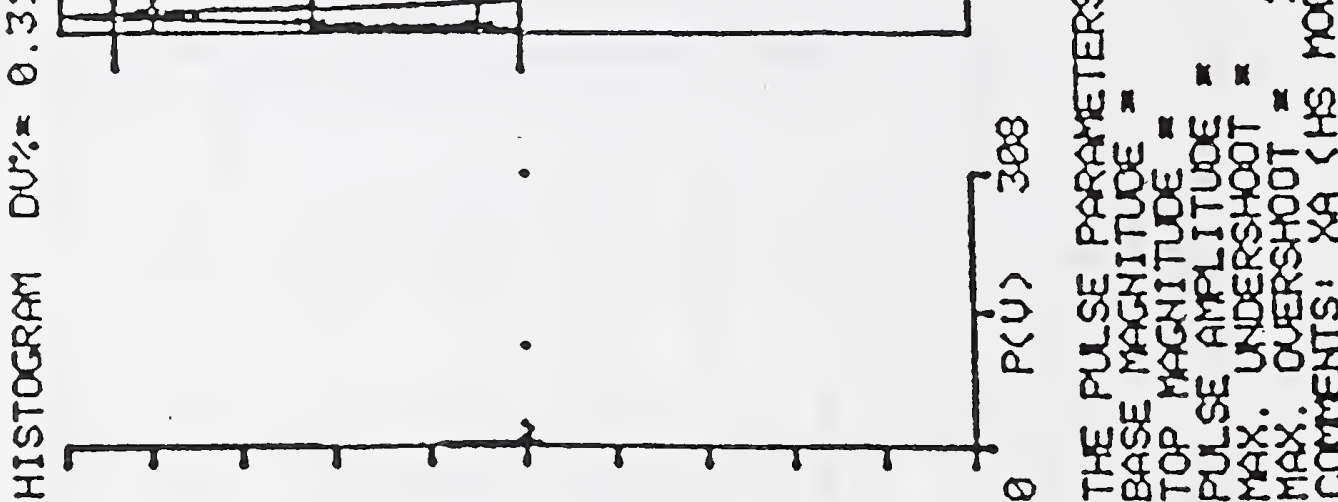

\&u 45 \& 5 F乐乐

a.

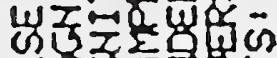

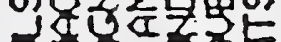

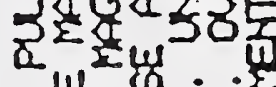

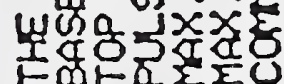

Figure 3.6 The impulse response and probability density histogram of the model for the Hewlett-Packard Model 1430B sampling head used in the NBS APMS. 


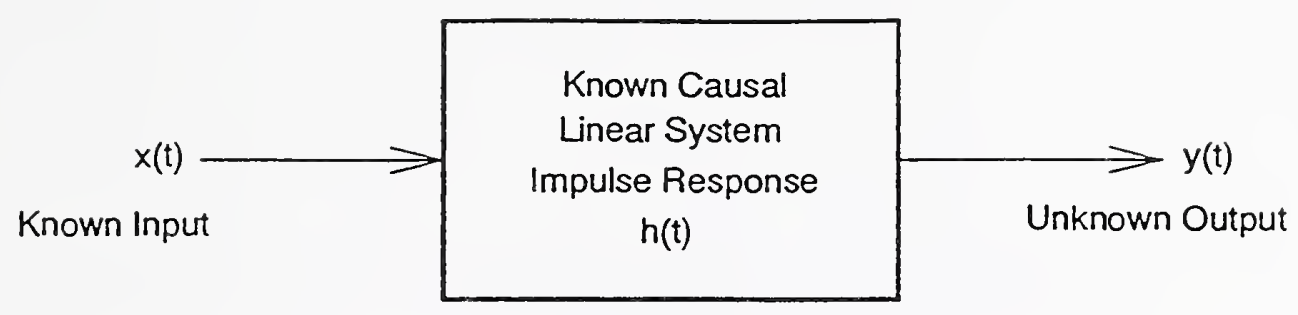

A. Convolution

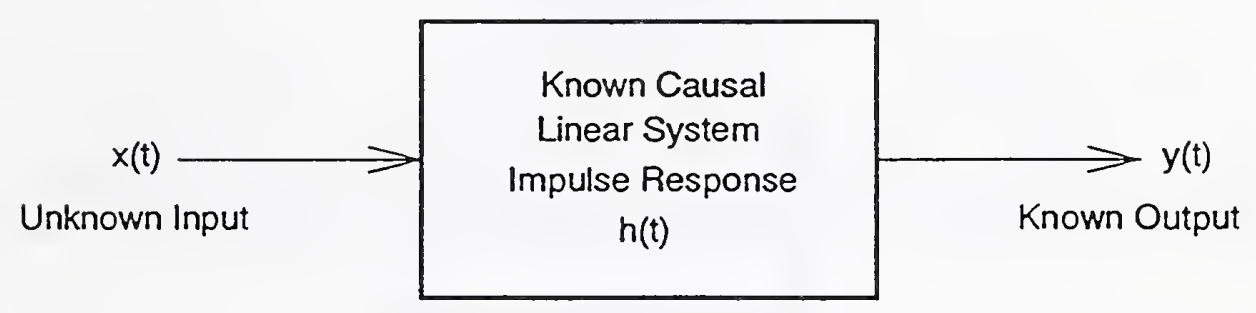

B. Deconvolution, Input Signal Unknown

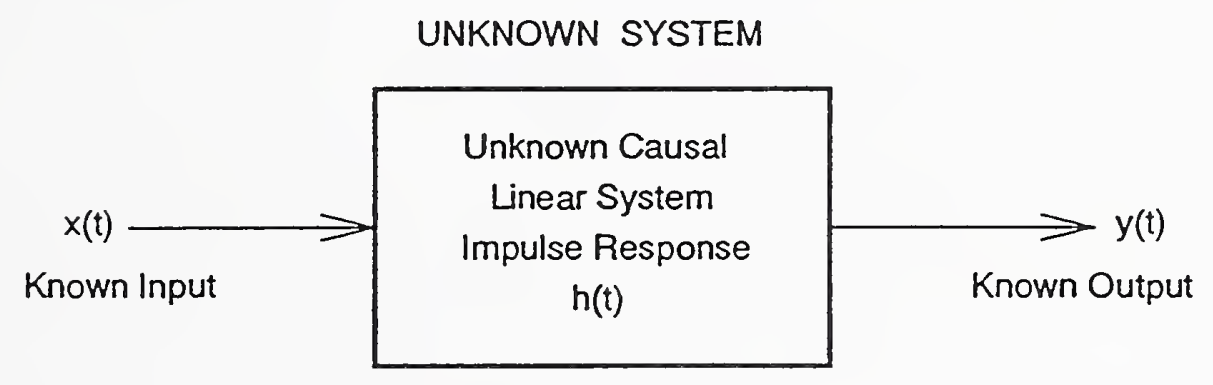

C. Deconvolution, System Impulse Response Unknown

Figure 3.7 Block diagram illustrating the convolution and deconvolution problems. 


$$
x(t)=x_{0}(t)+n_{x}(t) \longrightarrow h(t)=h_{0}(t)+n_{h}(t) \longrightarrow y(t)=y_{0}(t)+n_{y}(t)
$$

Figure 3.8 An actual physical measurement system. The actual variables, $x(t), h(t)$, and $y(t)$ differ from the conceptual ones by the respective departures $n_{x}(t), n_{h}(t)$, and $n_{y}(t)$. 


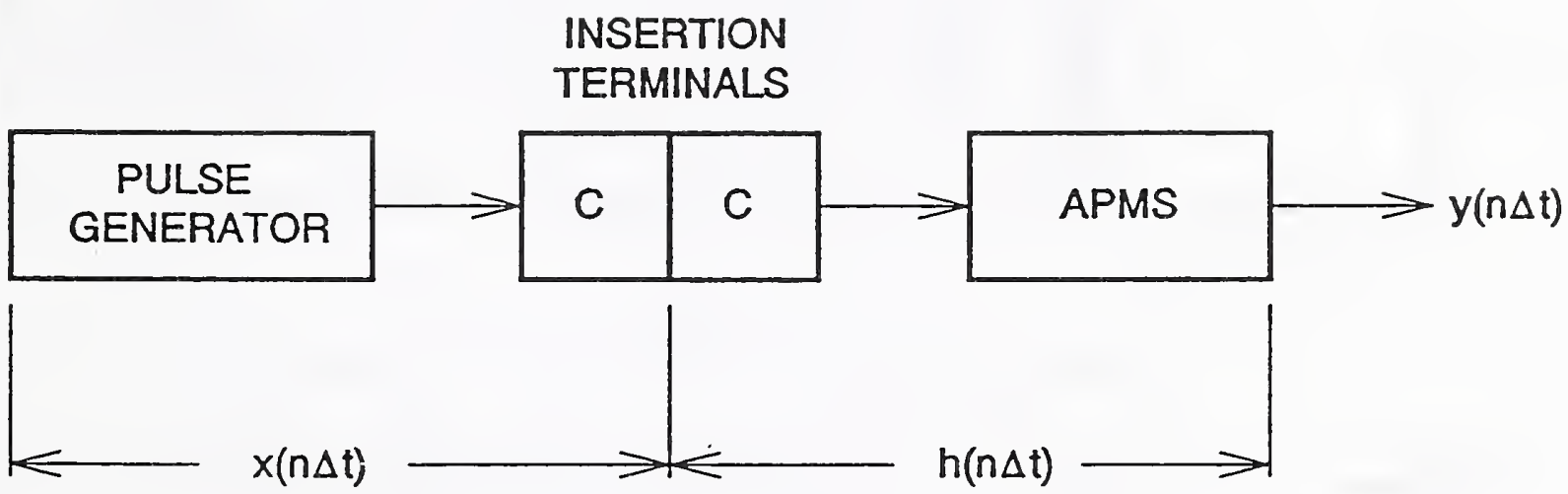

Figure 3.9 The pulse-generator measurement system. CC denotes the connector pair. In principle, the output $y(n \Delta t)$ is equal to the convolution of the pulse being measured $x(n \Delta t)$ with the impulse response of the measurement system $h(n \Delta t)$. 

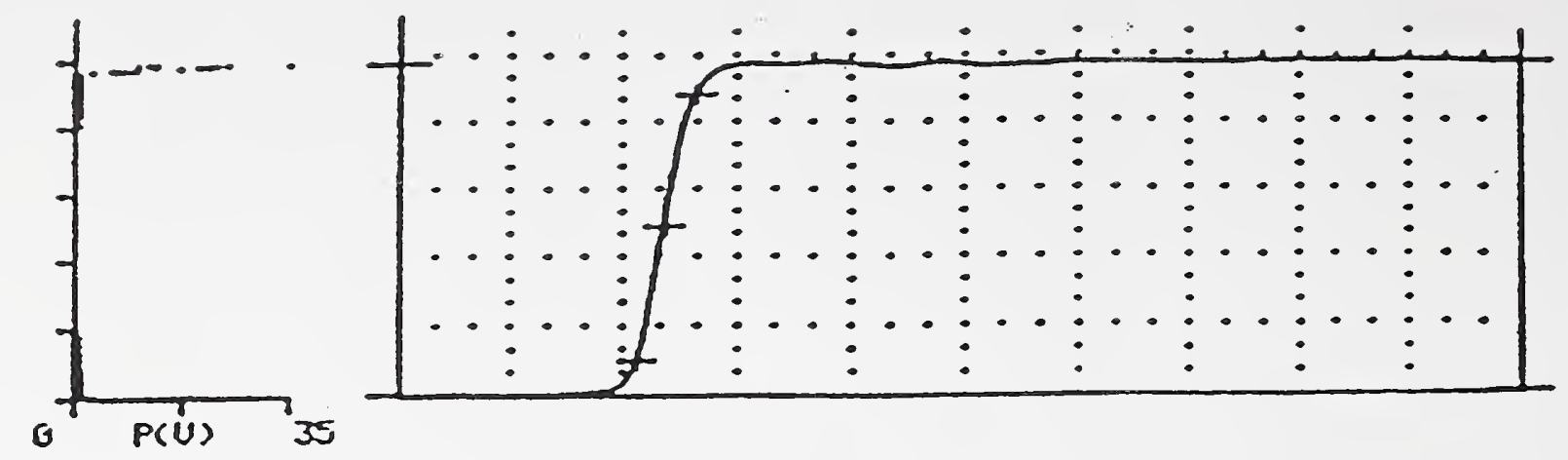

(A)
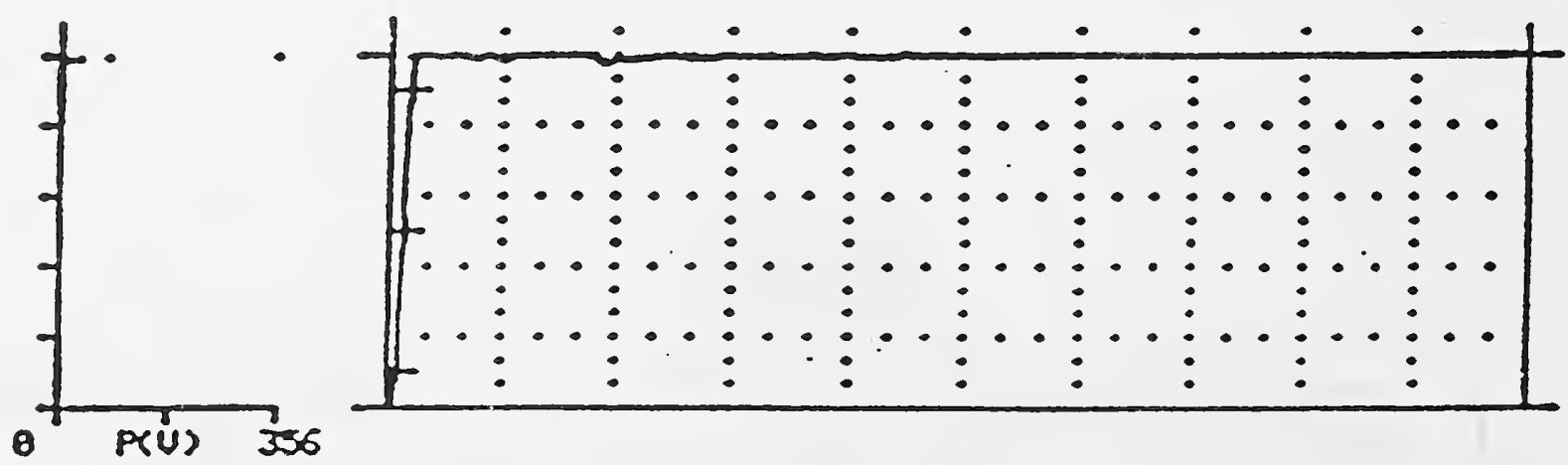

(B)
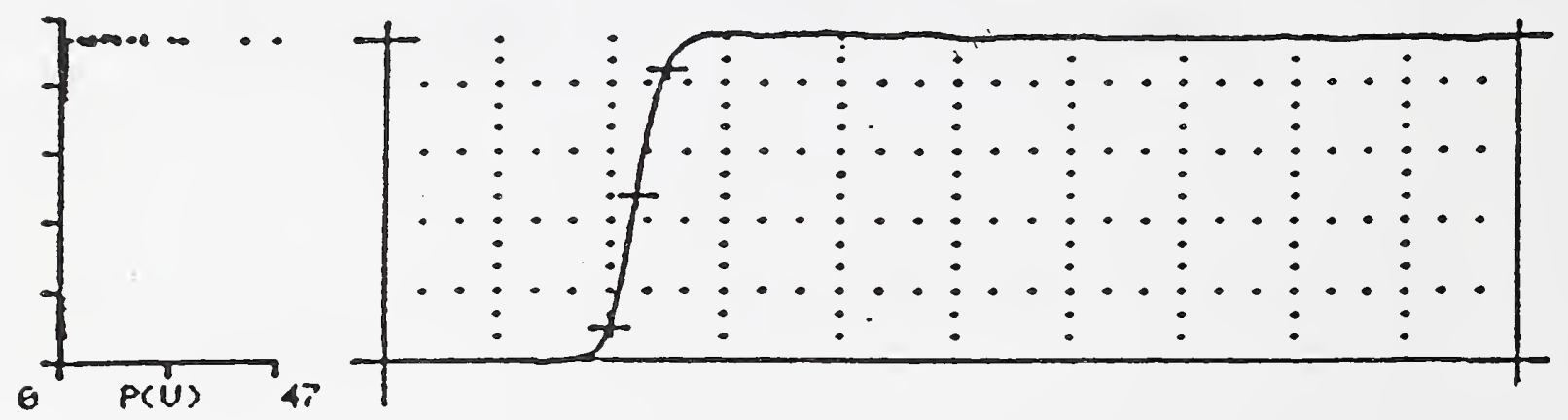

(C)

Figure 3.10 A pulse generator problem. (A) The measured output waveform $y(n \Delta t)$ from an NBS 100 ps transition duration standard; TD $=103.6 \mathrm{ps}$. (B) The sampler estimated step response $h_{0}(n \Delta t)$; TD $=20.85 \mathrm{ps}$. (C) The deconvolved estimate $\left(\gamma_{0}=1.35 \times 10^{5}\right)$ of the true output waveform from the standard $\left[d_{x}(n \Delta t)\right]_{\mathrm{y}} ; \mathrm{TD}=97.4 \mathrm{ps} . \mathrm{N}=512$ points, vertical scale factors for $(\mathrm{A})$ and $(\mathrm{C})$ are $50 \mathrm{mV} / \mathrm{div}$, for (B), $200 \mathrm{mV} / \mathrm{div}$, and time scale factor for all is $200 \mathrm{ps} / \mathrm{div}$. 
INSERTION

\section{TERMINALS}
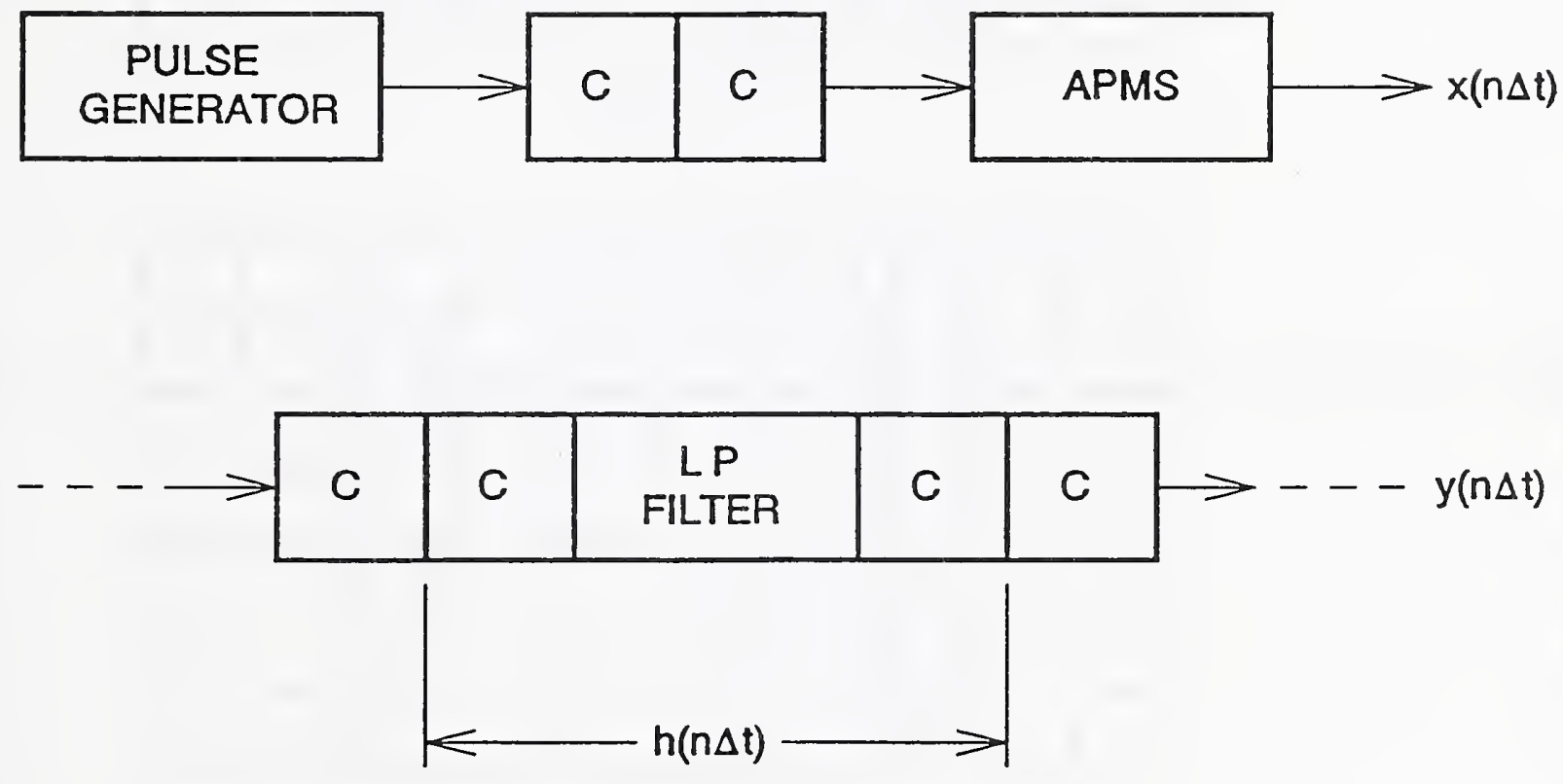

Figure 3.11 The low-pass filter measurement system. CC denotes the connector pair. The measured waveforms are shown as $x(n \Delta t)$ and $y(n \Delta t)$. In principle, the response $y(n \Delta t)$ is equal to the convolution of the reference waveform $x(n \Delta t)$ with the impulse response $h(n \Delta t)$ of the filter being measured. 


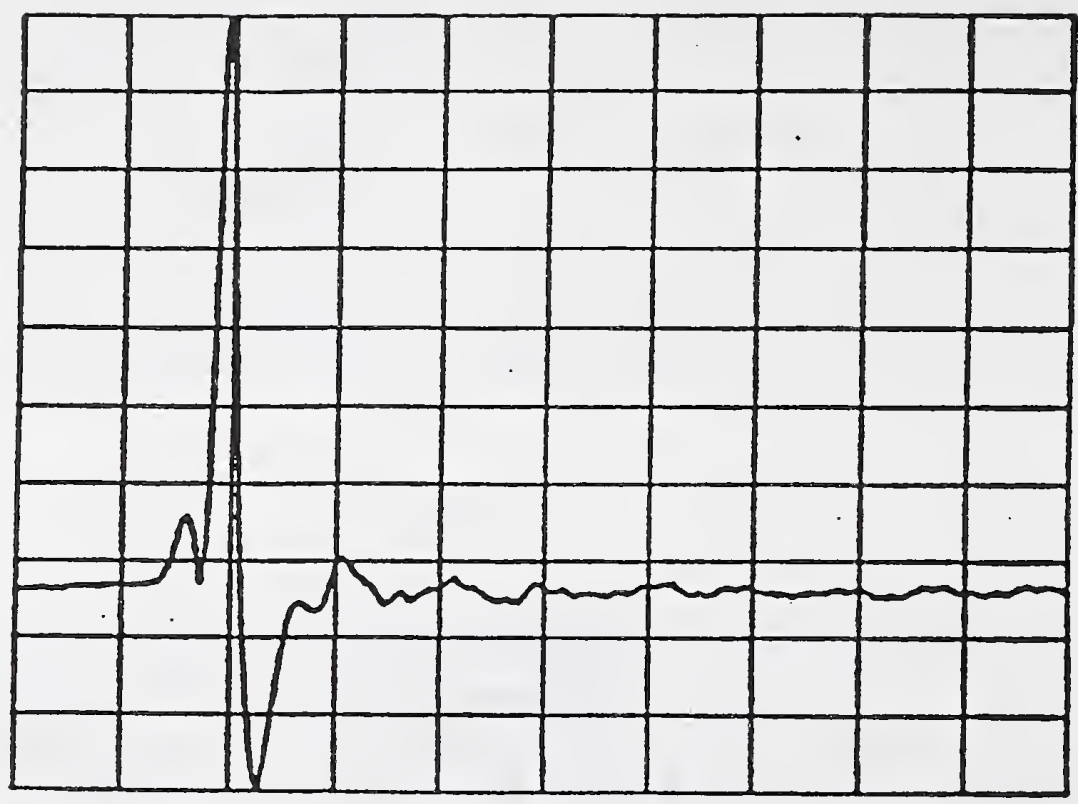

(A)

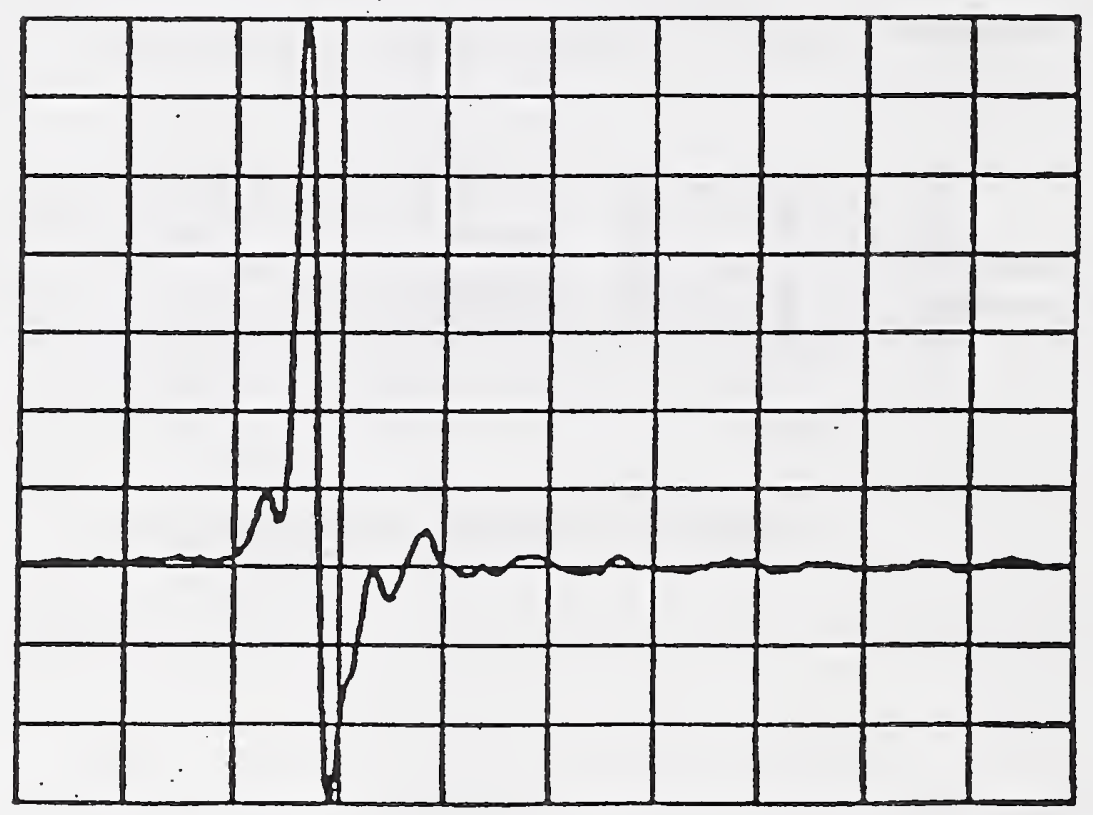

(B)

Figure 3.12 A low-pass filter problem. (A) The measured reference waveform $x(n \Delta t)$; $V_{\text {pk-pk }}=25.8 \mathrm{~V}$. (B) The measured response waveform $y(n \Delta t)$ for a $3.5 \mathrm{GHz}$ low-pass filter; $\mathrm{V}_{\text {pk-pk }}=23.7 \mathrm{~V}$. For both waveforms, $\mathrm{N}=512$ points, time scale factor is $1 \mathrm{~ns} / \mathrm{div}$, and number of waveform ensemble averages is 100 . 


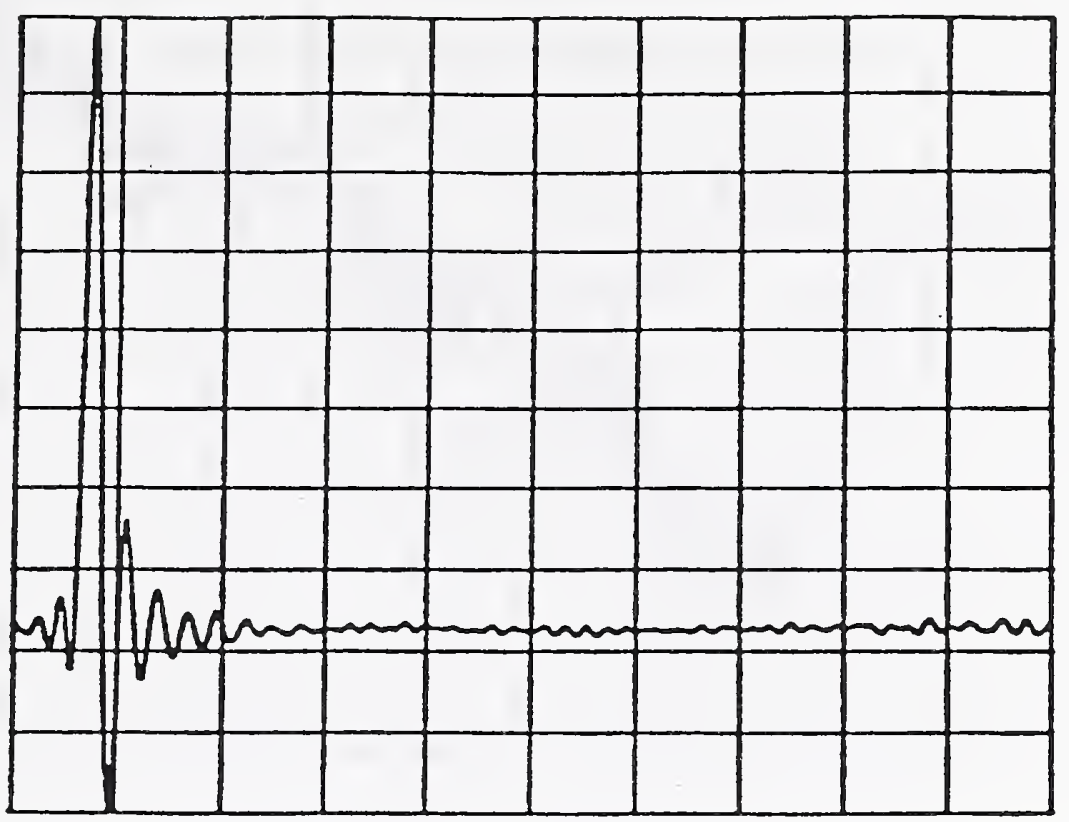

(A)
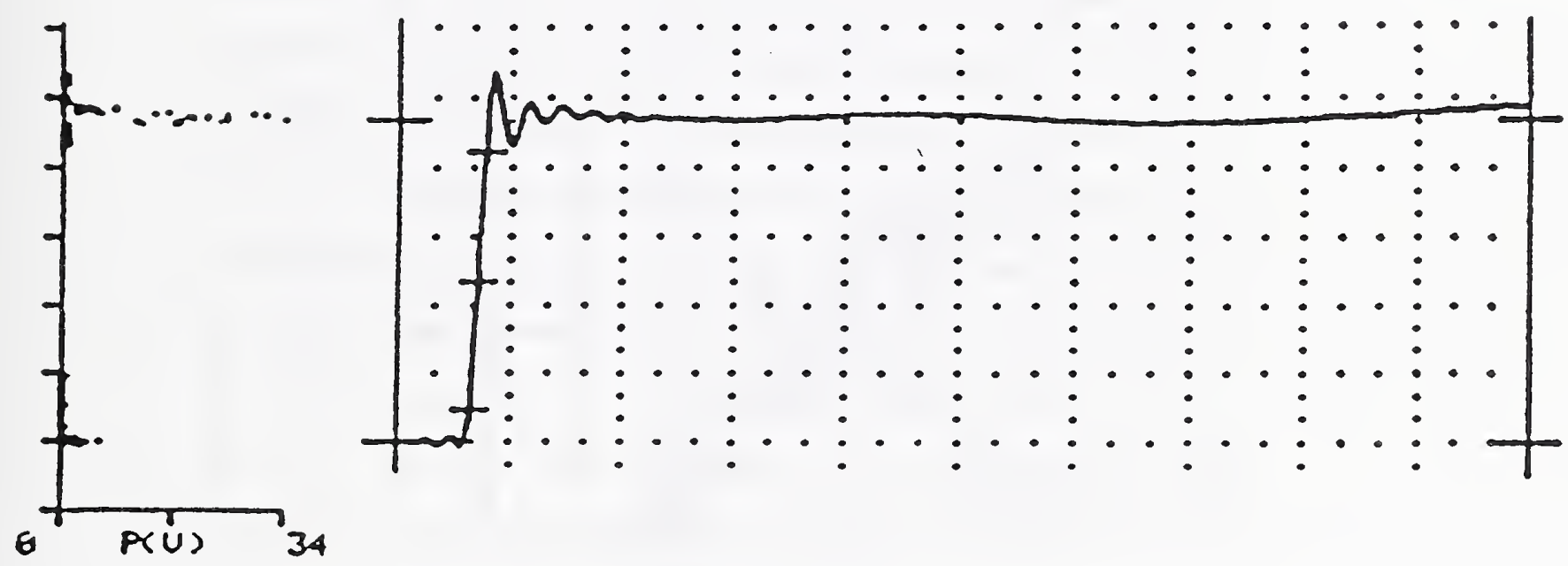

(B)

Figure 3.13 A low-pass filter problem. (A) The deconvolved estimate $\left(\mathrm{Y}_{0}=9 \times 10^{8}\right)$ of the $3.5 \mathrm{GHz}$ low-pass filter impulse response $\left[d_{h}(n \Delta t)\right]_{Y 0}$. (B) The estimated step response of the filter (integral of the impulse response); TD $=124.8 \mathrm{ps}$. Vertical scale factor for $(A)$ is arbitrary and for $(B)$ is $200 \mathrm{mV} /$ div; time scale factor is $1 \mathrm{~ns} / \mathrm{div}$ for both waveforms. 


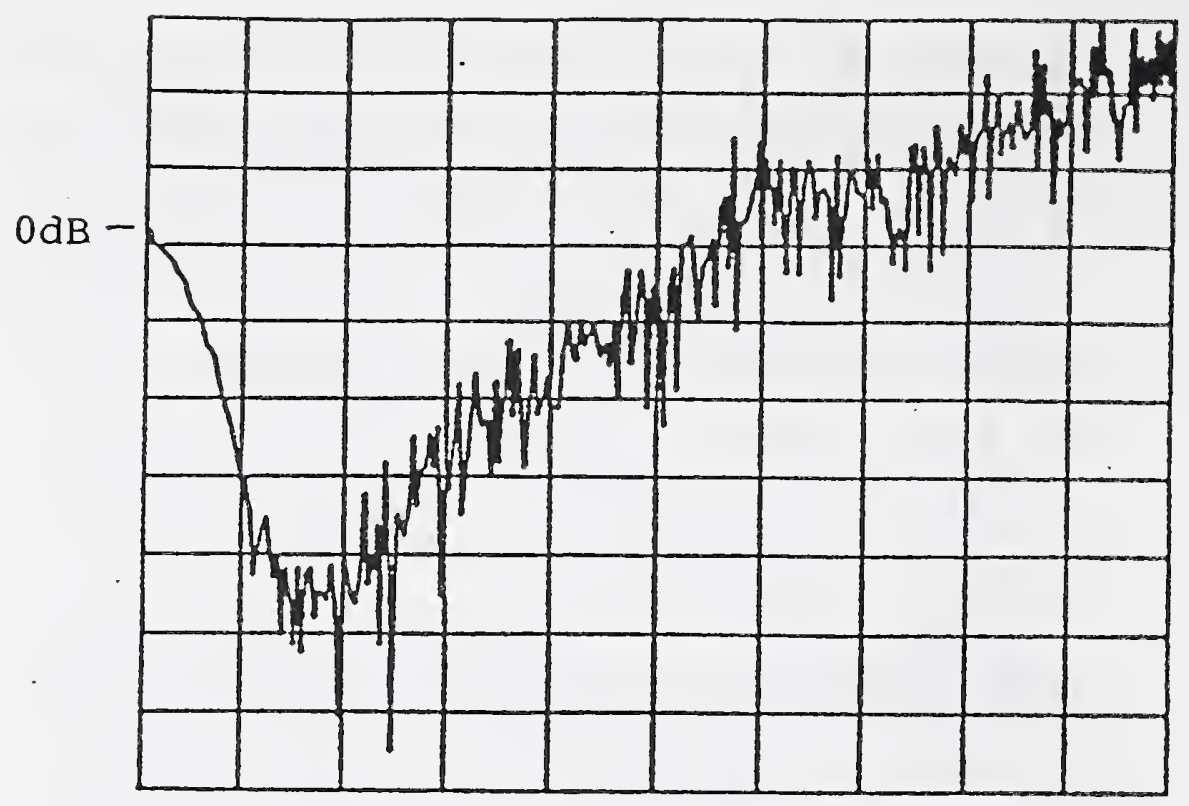

(A)

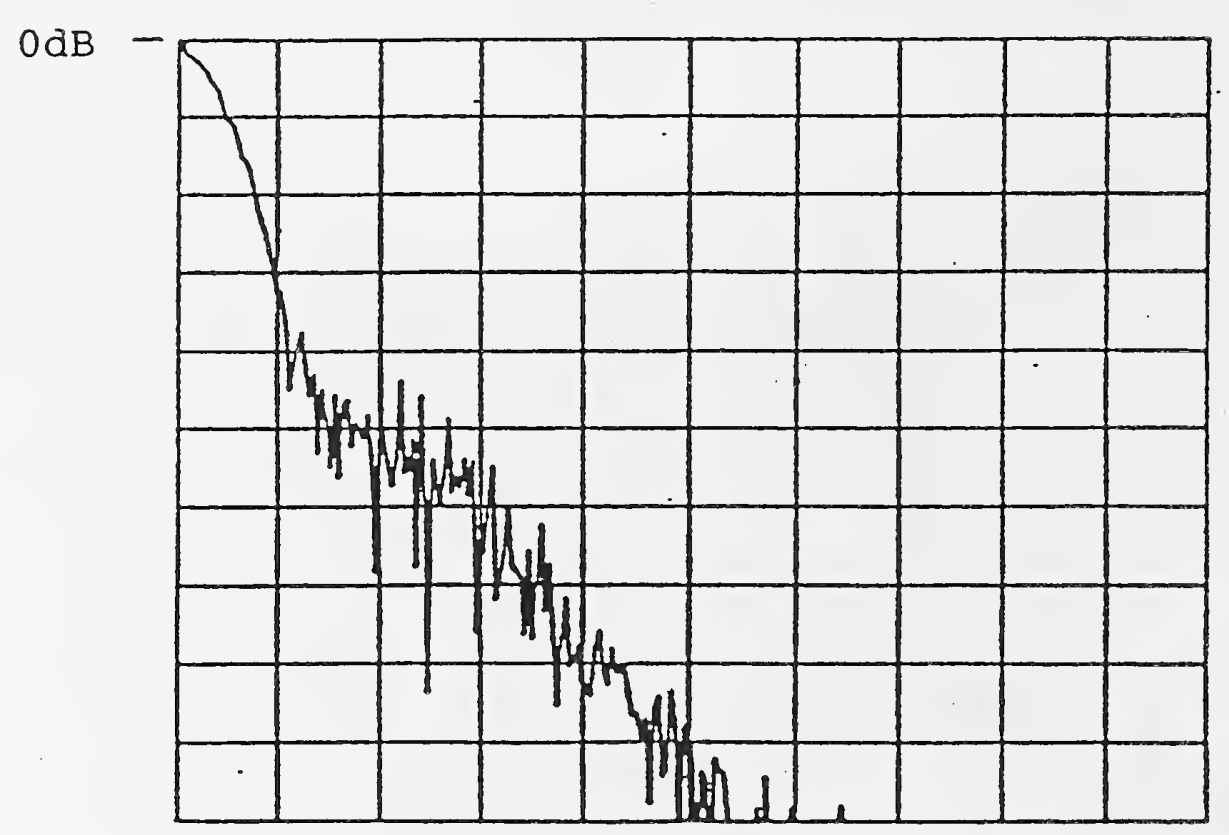

(B)

Figure 3.14 A pulse generator problem. (A) The spectrum magnitude $\left|D_{x}(k \Delta \Omega)\right|$ of the deconvolved estimate of the true output waveform $d_{x}(n \Delta t)$ with no filtering, $Y=0$. (B) The spectrum magnitude of the deconvolved estimate of the true output waveform with optimal filtering, $y=1.35 \times 10^{5}$. For both spectra, vertical scale factor is $10 \mathrm{~dB} / \mathrm{div}$ and frequency scale factor is $12.5 \mathrm{GHz} / \mathrm{div}$. (See Fig 3.10 for associated time-domain waveform plots.) 


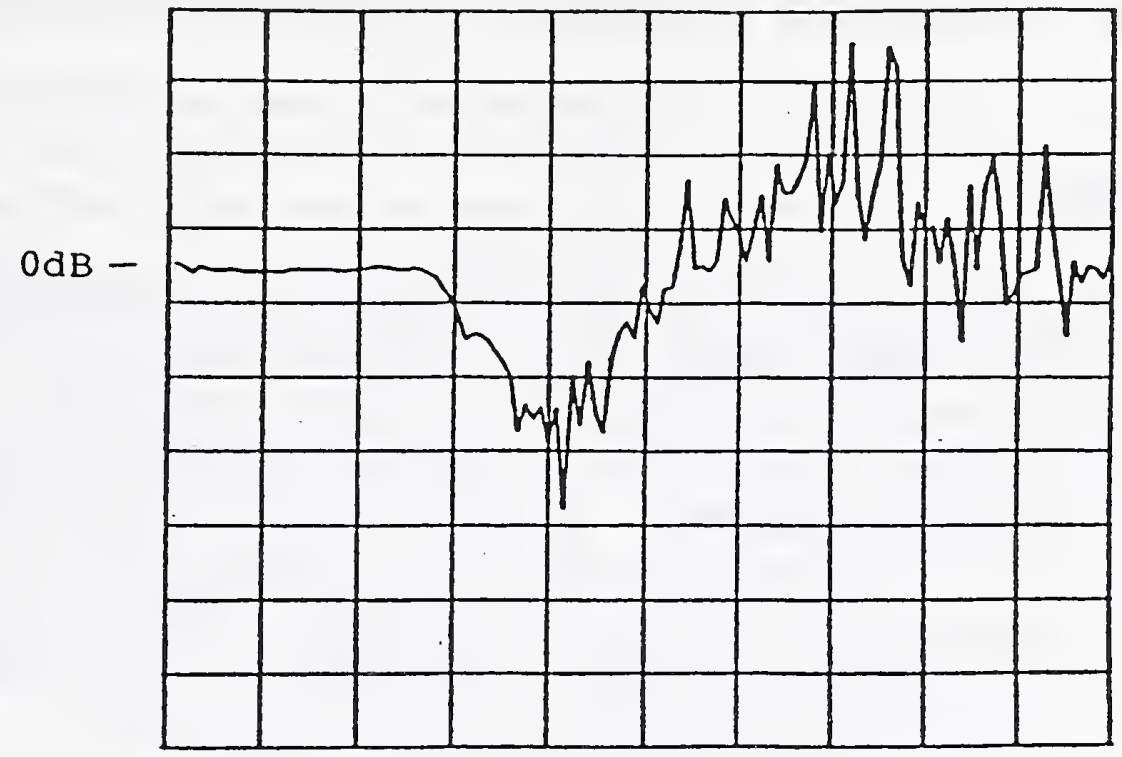

(A)

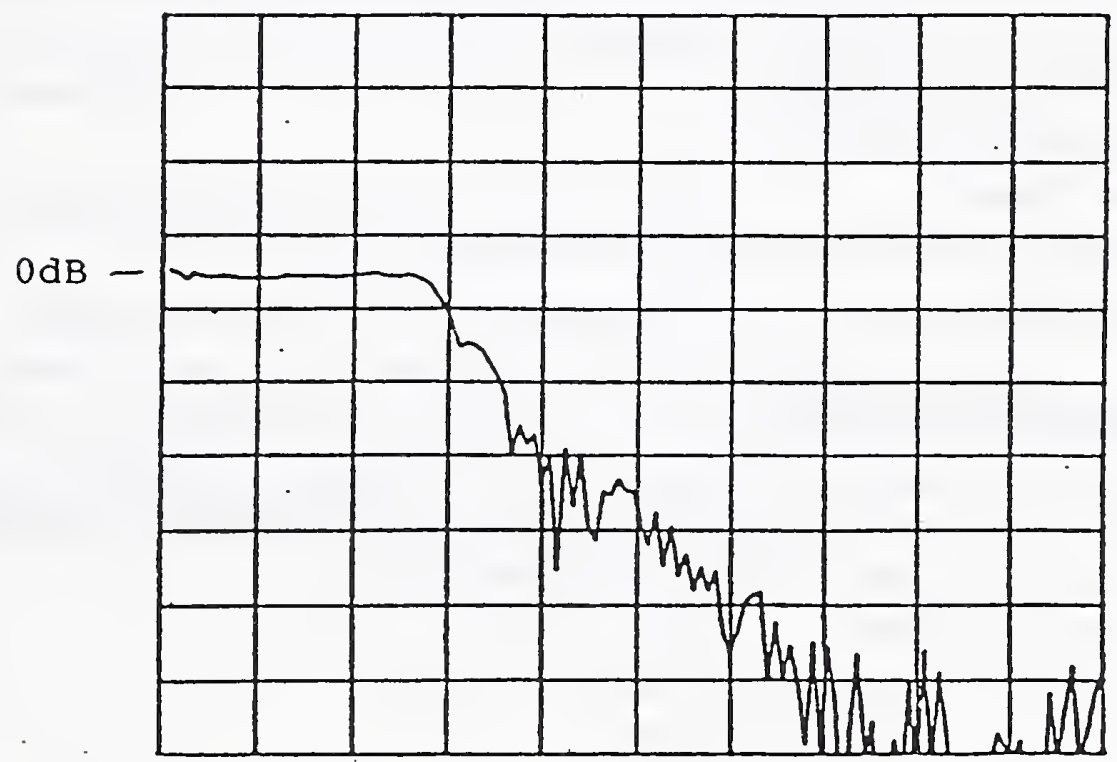

(B)

Figure 3.15 A low-pass filter problem. (A) The spectrum magnitude $\left|D_{h}(k \Delta \Omega)\right|$ of the deconvolved estimate of the impulse response $d_{h}(n \Delta t)$ of the $3.5 \mathrm{GHz}$ lowpass filter with no filtering, $y=0$. (B) The spectrum magnitude of the deconvolved estimate of the impulse response of the $3.5 \mathrm{GHz}$ filter with optimal filtering, $y=9.0 \times 10^{8}$. For both spectra, vertical scale factor is $10 \mathrm{~dB} / \mathrm{div}$ and frequency scale factor is $1.25 \mathrm{GHz}$ /div. (See Fig 3.13 for associated time-domain waveform plots.) 


\section{Pulse Transition Duration Transfer Standard}

\subsection{Introduction}

In the late 70's, NBS developed a reference pulse (waveform) generator for use as a transfer standard for transition duration (10\% to $90 \%$ TD). The generator consists of a tunnel-diode step generator with a TD of about $25 \mathrm{ps}$ driving a low-pass filter. Three filters are available for $T D=50,100$, and 200 ps. The output is a clean, Gaussian, step-like pulse. The low-pass filters, of NIST design, are $30 \mathrm{~cm}$ in length, $7 \mathrm{~mm}$ in diameter, coaxial lines filled with a lossy, Debye-type, liquid dielectric. The mathematical model describing these low-pass filters is quite accurate and the necessary parameters for the model can be obtained from independent measurements. The complete available output waveform into a matched load can be predicted with less than $\pm 1.5 \%$ uncertainty.

The remainder of this Chapter is divided into five sections whose order and titles are as follows:

1. Objective and Concept;

2. Selection of the Filter;

3. Filter Model;

4. Definition and Prediction of the Available Waveform;

5. Hardware.

\subsection{Objective and Concept}

The objective was to develop a pulse generator suitable for use as a transfer standard for transition duration. The specific requirements were:

1. step-like pulse;

2. transition duration of the order of $100 \mathrm{ps}$;

3. smooth, Gaussian transition;

4. clean baseline and topline;

5. physical model for predicting the available waveform.

The concept chosen for the implementation was to use a tunnel diode to generate a rapidly rising ( $20 \mathrm{ps})$, but not necessarily well known, step-like pulse. The fast transition is then slowed down using a known low-pass filter. If the step response of the filter is ten or more times slower than the driving pulse, then the transition duration of the output pulse from the composite generator is specified to within $0.5 \%$ or better by the step response of the filter alone. 


\subsection{Selection of the Filter}

Most commercial, low-pass filters are unsuitable for this application. They are typically designed to meet frequency domain specifications using Butterworth, Chebyshev, etc., designs. Their time-domain step response usually is corrupted with overshoot and ringing [31], as illustrated in Fig 4.1. Some pulse equipment manufacturers in the past have made low-pass filters that were designed for a clean time-domain response. Some of these commercial filters were found to have a serious defect. The leading edge of their step responses were distorted with many small transitions, as shown in Fig 4.2. These small transitions were readily observable using a nominal $20 \mathrm{ps}$ tunnel-diode pulse generator and a nominal 20 ps sampling oscilloscope. They were caused by multiple paths and reflections from the various elements used to construct the filter. Another filter that had been proposed [32] was a long length of coaxial cable. The skin effect gives a loss proportional to $\sqrt{\mathrm{f}}$. The pulse response of a cable is usually quite clean, but it is also unsuitable due to its extremely long "dribble-up," as shown in Fig 4.3.

It was found that a suitable time domain filter for transition durations from 50 ps to 500 ps could be obtained from a coaxial transmission line with a lossy dielectric and low conductor losses [32].

\subsection{The Filter Model}

A lossy, distributed-element filter was chosen for use as a transfer standard [33]. It consists of a coaxial transmission line filled with a lossy liquid dielectric. The dielectric is a dilute solution of 2-heptanone in heptane. The molecules of the heptane solvent are non-polar and essentially lossless. The 2-heptanone solute has a polar molecular structure and obeys the Debye dielectric equations [34],

$$
\epsilon(s)=\frac{\epsilon(0)-\epsilon(\infty)}{1+s T},
$$

where $s$ is the complex frequency (Laplace transform variable),

$$
s=\sigma+j \omega .
$$

$\epsilon(0)$ is the dielectric constant at zero frequency, $\epsilon(\infty)$ is the dielectric constant at infinite frequency, and $\mathrm{T}$ (tau) is the relaxation time constant of the polar solution. On the frequency axis, $\sigma=0$, and, 


$$
\epsilon(j \omega)=\epsilon(\infty)+\frac{\epsilon(0)-\epsilon(\infty)}{1+j \omega T}
$$

or,

$$
\epsilon(j \omega)=\frac{\epsilon(\infty) \omega^{2} \mathrm{~T}^{2}+\epsilon(0)}{1+\omega^{2} \mathrm{~T}^{2}}-j \omega \mathrm{T} \frac{\epsilon(0)-\epsilon(\infty)}{1+\omega^{2} \mathrm{~T}^{2}}
$$

Simplifying the notation,

$$
\epsilon(j \omega)=\epsilon^{\prime}(\omega)-j \epsilon^{\prime \prime}(\omega)
$$

The behavior versus frequency of the real and imaginary parts, $\epsilon^{\prime}(\omega)$ and $\epsilon^{\prime \prime}(\omega)$, of the dielectric function $\epsilon(j \omega)$ are shown in Fig 4.4 .

Explicitly, the dielectric function, $e(j \omega)$, is a function of the molal concentration, $m$, of the 2-heptanone solute in the heptane solvent,

$$
\epsilon(j \omega, m)=\epsilon(\infty)+\frac{\epsilon(0, m)-\epsilon(\infty)}{1+j \omega T(m)} .
$$

$\epsilon(\infty)$ is the dielectric constant of the non-polar heptane solvent, and is also equal to the infinite frequency dielectric constant of 2-heptanone. At very high frequencies the dielectric properties of 2-heptanone are determined by its electronic polarization which is the same as that for heptane. $\Theta(0, m)$ is the static dielectric constant which is strongly dependent upon the polar (molecular) properties of the 2-heptanone solute.

Fig 4.5 shows the per-unit-length equivalent circuit of a coaxial cable with a Debye-type dielectric. $s$ is the complex frequency, $L$ is the normal series inductance, $k$ is the skin effect constant for the metal conductors, and $C_{1}+C_{2}$ is the normal low-frequency shunt capacitance proportional to $\epsilon^{\prime}(0, m)$.

The transfer function, $H(s)$, of a transmission line of length $\ell$ terminated in its characteristic impedance, $Z_{o}(s)$, is given by, 


$$
H(s)=\frac{V_{\text {out }}(s)}{V_{\text {in }}(s)}=e^{-\gamma(s) l},
$$

where $Y(s)$ (gamma of $s$ ) is the propagation function. $Y(s)$ and $Z_{o}(s)$ are defined in terms of the per-unit-length series impedance, $Z(s)$, and the per-unit-length shunt admittance, $Y(s)$ (wye of s), as follows.

$$
\begin{aligned}
& Y(s)=\sqrt{Z(s) Y(s)}, \\
& Z_{0}(s)=\sqrt{\frac{Z(s)}{Y(s)}}, \\
& Z(s)=s L+k \sqrt{s},
\end{aligned}
$$

and,

$$
Y(s)=s C_{1}+\frac{s C_{2}}{1+s T} .
$$

By varying the molal concentration, $m, \epsilon^{\prime}(0, m)$ and $T(m)$ are varied; and as a result, $Z_{o}(s)$ and $\gamma(s)$ can be adjusted. The effect of varying concentration on the step response transition duration is vividly demonstrated in Fig 4.6.

Using Eq. (4.7), the complete step response, Fig 4.7, of a Debye line filter terminated in $Z_{o}(s)$ can be predicted. Note the clean, Gaussian-like transition. The various parameters can be determined from independent measurements; $k$ is a function of the line diameters and the metal conductivity, $\epsilon^{\prime}(0)$ is determined by $1 \mathrm{kHz}$ capacitance-bridge measurements, $\epsilon^{\prime}(\infty)$ is determined by the index of refraction of heptane, $L$ is determined by the line diameters, and $\mathrm{T}(m)$ is determined by an iterative procedure using Eq. (4.7) and frequency and time measurements [35]. The complete model of the actual filters also includes equivalent circuits for the non-perfect connectors, Fig 4.8. 


\subsection{Definition and Prediction of the Available-Pulse (Waveform)}

The term available pulse is defined as the voltage, $e_{\mathrm{a}}(t)$, produced by a reference pulse generator across a specified load. A reference pulse generator is a generator that produces a pulse of known mathematical (or numerical) form [33].

The terms pulse and waveform are not synonymous, the former being the physical electrical signal being measured, while the latter is the measured manifestation of the pulse; in short, the measurement process distorts the pulse; and the two representations must carry separate designations. Consequently, if $e_{a}(t)$ is a pulse in an operating circuit, then its designation in a circuit diagram is also $e_{\mathrm{a}}(t)$; however, when a measurement is performed to determine $e_{a}(t)$, the designation of the measured $e_{a}(t)$ is $e_{a m}(t)$ which is the waveform. It might be argued that the effects of the measurement have been removed by deconvolution, but the question remains, how well? Therefore, it is appropriate to allow for a difference by designating one as the pulse $e_{\mathrm{a}}(t)$ and the other as the waveform $e_{\mathrm{am}}(t)$.

One historical note, in [33] the term waveform is used generally but such use does not invalidate the concept of a reference time-domain generator and its associated timedomain available output.

The procedure for modeling the available pulse, $e_{a}(t)$, is based upon the circuits shown in Fig 4.9. The $50 \Omega$ source and load impedances are maintained for specified time windows (say 2 nanoseconds) by precision air-dielectric lines [36]. $H(s)$ is the overall transfer function of the oscilloscope system, that is, the transfer function from the input connector of the load precision line to the oscilloscope display (sampling channel analog output). The product $N_{3}(s) T(s) N_{4}(s)$ is the overall transfer function of the low-pass filter when operating into a $50 \Omega$ load and being driven by a $50 \Omega$ source. Consequently, the generator voltage, $E_{g}(s)$, is obtained from the observed voltage, $E_{1}(s)$, as,

$$
E_{1}(s)=E_{g}(s) H(s) / 2,
$$

which yields,

$$
E_{g}(s)=2 E_{1}(s) / H(s) \text {. }
$$

The available pulse, $E_{\mathrm{a}}(s)$, is obtained from the observed voltage, $E_{2}(s)$, as,

$$
E_{2}(s)=E_{g}(s) N_{3}(s) T(s) N_{4}(s) H(s) / 2 \text {, }
$$


and,

$$
E_{2}(s)=E_{a}(s) H(s)
$$

Therefore,

$$
E_{a}(s)=E_{2}(s) / H(s)
$$

and,

$$
E_{a}(s)=E_{g}(s) N_{3}(s) T(s) N_{4}(s) / 2
$$

The time domain voltages corresponding to $E_{g}(s)$ and $E_{\mathrm{a}}(s)$ are,

$$
e_{g}(t)=\mathscr{L}^{-1}\left[2 E_{1}(s) / H(s)\right]
$$

and,

$$
e_{a}(t)=\mathscr{L}^{-1}\left[E_{2}(s) / H(s)\right]
$$

where $\mathscr{L}^{-1}$ is the inverse Laplace transform operator.

The voltages $e_{\mathrm{g}}(t)$ and $e_{\mathrm{a}}(t)$ are not observable voltages, only their counterparts $e_{1}(t)$ and $e_{2}(t)$ are. Consequently, the waveforms for $e_{g}(t)$ and $e_{a}(t)$ are accessible only through deconvolution, the removal of the effects of $H(s)$ in Eqs. (4.18) and (4.19). Theoretically, to obtain $e_{g}(t)$ and $e_{a}(t), H(s)$ must be known so that $E_{1}(s)$ and $E_{2}(s)$ may be divided by $H(s) / 2$ to obtain $E_{\mathrm{g}}(s)$ and $E_{\mathrm{a}}(s)$, respectively.

When $H(s)$ is deconvolved in a measurement procedure, the resultant quantities are the waveforms $e_{\mathrm{gm}}(t)$ and $e_{\mathrm{am}}(t)$ and not the pulses $e_{\mathrm{g}}(t)$ and $e_{\mathrm{a}}(t)$. A typical set of deconvolved waveforms is shown in Fig 4.10 where the tunnel-diode generator waveform, $e_{\mathrm{gm}}(t)$, is shown along with the $e_{\mathrm{am}}(t)$ waveforms for the three transition durations: $50 \mathrm{ps,}$ $100 \mathrm{ps}$, and $200 \mathrm{ps}$.

The available pulse, $e_{a}(t)$, is the predicted pulse generator output across $50 \Omega$. For example, Fig 4.11 shows the predicted, available, reference pulse into a $50 \Omega$ load from the tunnel-diode/100 ps low-pass-filter combination. It was obtained by convolving the measured tunnel diode waveform, $e_{\text {gm }}(t)$, with the filter transfer function. The tunnel diode 
waveform, Fig 4.10a, was first obtained by deconvolution of the measured tunnel diode waveform and a model of the sampling oscilloscope used to make the measurement. The deconvolution techniques and sampler models were those discussed in Section 3.2.2 (from [12] and [28]). The low-pass filter parameters were determined through independent timeand frequency-domain measurements as described in [33].

Fig 4.12 demonstrates the agreement between theory and experiment. The solid curve is the mathematically predicted pulse, $e_{\mathrm{a}}(t)$, (tunnel-diode/100 ps filter), while the dotted line is the actual measured waveform. It is meaningful to compare two similar waveforms to determine the resultant difference [37]. The difference between these two waveforms is shown for a 2 ns time window. The RMS difference was $0.4 \%$ while the maximum difference was $1.4 \%$.

The prediction for the pulse, $e_{\mathrm{a}}(t)$, was based upon $e_{\mathrm{gm}}(t)$, the measured value of $e_{g}(t)$. Because $e_{g m}(t)$ is equal to the convolution of $e_{g}(t)$ with the oscilloscope-system impulse response, $h(t)$, in the strictest sense $e_{\mathrm{a}}(t)$ is not a fully predicted result. When $e_{\mathrm{gm}}(t)$ is used in place of $e_{g}(t)$, the error due to estimating $e_{g}(t)$ from $e_{g m}(t)$ is not included in the predicted result.

As defined in Sections 3.5.4 and 3.5.5, the deconvolution error is small and affects the transition duration in such a way that the true transition duration, $T D_{R}$, is given by,

$$
T D_{R}=T D_{o} \frac{f_{o}}{f_{R}}
$$

where $T D_{0}$ and $f_{0}$ are the transition duration for $e_{\mathrm{gm}}(t)$ and its spectral $3 \mathrm{~dB}$ frequency, respectively. $f_{R}$ is the $3 \mathrm{~dB}$ spectral frequency for $e_{\mathrm{g}}(t) ; f_{R}$ is an observable even though $e_{g}(t)$ is not due to the noise which makes $e_{g}(t)$ unstable. Consequently, $e_{a}(t)$ should be calculated using an effective $e_{g}(t)$ constructed from $e_{g m}(t)$ by scaling $E_{g m}(j \omega)$ so that its spectral $3 \mathrm{~dB}$ frequency is increased from $f_{o}$ to $f_{R}$.

\subsection{Hardware}

Fig 4.13 is a photograph of an NBS Debye line low-pass filter with a tunnel diode step generator attached. Also shown in the figure is the temperature controller. Three different filters have been designed and developed for transition durations of $50 \mathrm{ps}, 100 \mathrm{ps}$, and 200 ps. A $30 \mathrm{~cm}, 7 \mathrm{~mm}$-diameter coaxial air line was used for each filter structure. Different molal solutions of 2-heptanone in heptane were used in each of the three models, 
and $k$, the center conductor diameter, was varied to maintain a $50 \Omega$ impedance in each model. Modified APC-7 connectors were used; the center-conductor support bead of each connector was modified to provide a leak-proof seal. Table 4.1, on the next page, shows typical parameters for the three NBS pulse transfer standard models. (No estimates for the uncertainties associated with the data shown in table 4.1 are available.)

The dielectric characteristics of the 2-heptanone and heptane fluids used are quite temperature sensitive. The temperature coefficient of the transition duration is approximately $1 \% /{ }^{\circ} \mathrm{C}$. Thus, it was necessary to provide temperature regulation to better than $\pm 0.1^{\circ} \mathrm{C}$. The filters were designed to be operated at $30^{\circ} \mathrm{C}$ and were intended to be operated only in a standard laboratory environment. Heaters and temperature sensors are installed in the filter box. Also enclosed in the box is the necessary plumbing and valve for filling the line and an expansion bellows to compensate for fluid expansion and contraction due to temperature extremes encountered during shipping. The plumbing details are given in Appendix 1 while the electronic temperature regulator is described in Appendix 2.

The tunnel diode used to drive the filter was a Hewlett-Packard Model 1106A. The Hewlett-Packard Model 1105A bias supply and trigger pulse generator normally used to drive the HP-1106A tunnel diode were replaced by an improved NBS design. The NBS bias supply has the advantage that it automatically compensates the tunnel diode bias current for changing tunnel diode conditions such as temperature, various electrical loads ranging from a short to an open circuit and even using a different tunnel diode. The trigger-pulse circuits were designed so that they introduced negligible distortion to the tunnel diode step-like pulse as shown in Fig 4.10a. This was a considerable improvement over the commercial bias supply which added a $5 \%, 1 / 2$ ns linear ramp distortion prior to the tunnel diode abrupt transition. The NBS bias supply is described in Appendix 3. 
Table 4-1

Typical physical parameters of NBS pulse transfer standards.

\begin{tabular}{|c|c|c|c|}
\hline Parameter & 50 ps filter & $100 \mathrm{ps}$ filter & 200 ps filter \\
\hline $\begin{array}{l}\text { nominal transition duration } \\
@ 30^{\circ} \mathrm{C}(10 \% \text {-to- } 90 \%)(\mathrm{ps})\end{array}$ & 50 & 100 & 200 \\
\hline $\begin{array}{c}\text { nominal characteristic } \\
\text { impedance @ } 30^{\circ} \mathrm{C}(\Omega)\end{array}$ & 50. & 50 & 50 \\
\hline coaxial line length (mm) & 300 & 300 & 300 \\
\hline $\begin{array}{c}\text { coaxial outer conductor } \\
\text { inner diameter }(\mathrm{mm})\end{array}$ & 7.0 & 7.0 & 7.0 \\
\hline $\begin{array}{c}\text { coaxial inner conductor } \\
\text { outer diameter }(\mathrm{mm})\end{array}$ & 2.13 & 1.92 & 1.524 \\
\hline coaxial line conductivity (S) & $4.098 \times 10^{7}$ & $4.098 \times 10^{7}$ & $4.098 \times 10^{7}$ \\
\hline $\begin{array}{l}\text { liquid dielectric real relative } \\
\text { permittivity } \epsilon^{\prime}(0) @ 30^{\circ} \mathrm{C}\end{array}$ & 2.024 & 2.390 & 3.507 \\
\hline $\begin{array}{l}\text { liquid dielectric real relative } \\
\text { permittivity } \epsilon^{\prime}(\infty) @ 30^{\circ} \mathrm{C}\end{array}$ & 1.912 & 1.918 & 1.958 \\
\hline $\begin{array}{l}\text { liquid dielectric relaxation } \\
\text { time constant } \mathrm{T} @ 30^{\circ} \mathrm{C}(\mathrm{ps})\end{array}$ & 3.2 & 3.5. & 4.3. \\
\hline $\begin{array}{l}\text { liquid dielectric molal } \\
\text { concentration @ } 30^{\circ} \mathrm{C}\end{array}$ & 0.21 & 0.91 & 3.20 \\
\hline $\begin{array}{l}\text { coaxial line input connector } \\
\text { series inductance } L_{3}(p H)\end{array}$ & 61 & 50 & 40 \\
\hline $\begin{array}{l}\text { coaxial line input connector } \\
\text { shunt capacitance } C_{3}(\mathrm{fF})\end{array}$ & 67 & 66 & 69 \\
\hline $\begin{array}{l}\text { coaxial line output connector } \\
\text { series inductance } \mathrm{L}_{4}(\mathrm{pH})\end{array}$ & 170 & 130 & 85 \\
\hline $\begin{array}{l}\text { coaxial line output connector } \\
\text { shunt capacitance } \mathrm{C}_{4}(\mathrm{fF})\end{array}$ & 49 & 48 & 69 \\
\hline
\end{tabular}




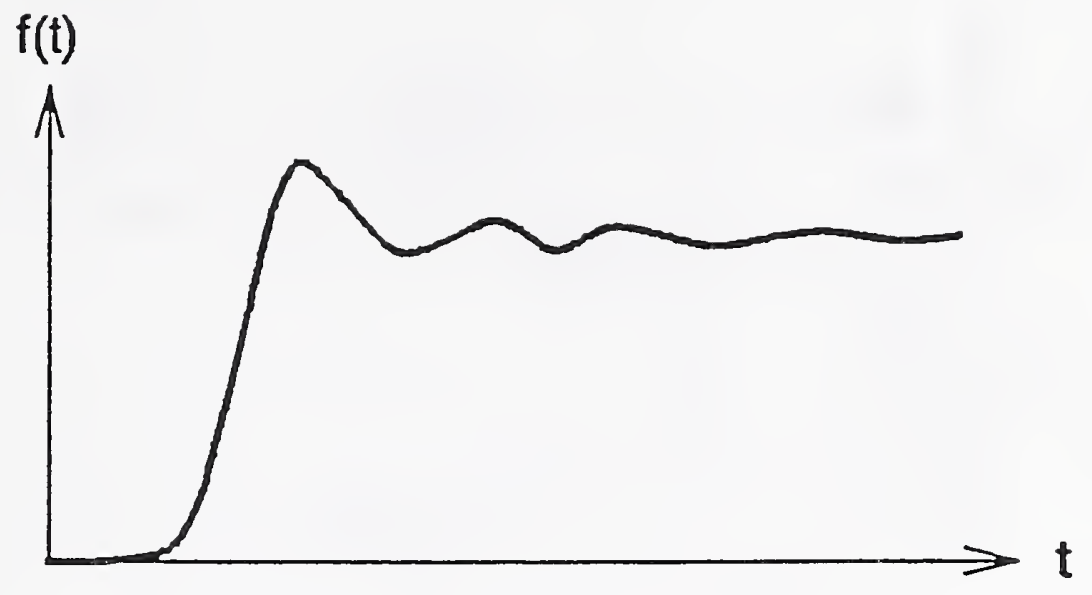

Figure 4.1 Typical step response $f(t)$ of a low-pass filter having a sharp cut-off frequency.

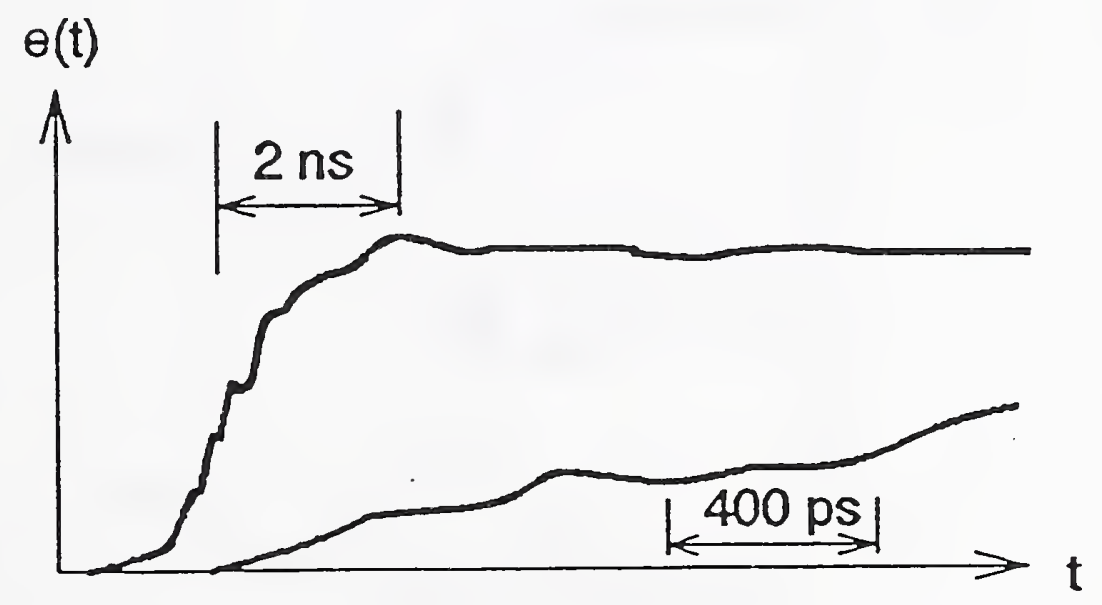

Figure 4.2 Commercial $2 \mathrm{~ns}$ transition duration standard. Note the small steps on the leading edge. The lower curve shows the leading edge on a faster time scale. 


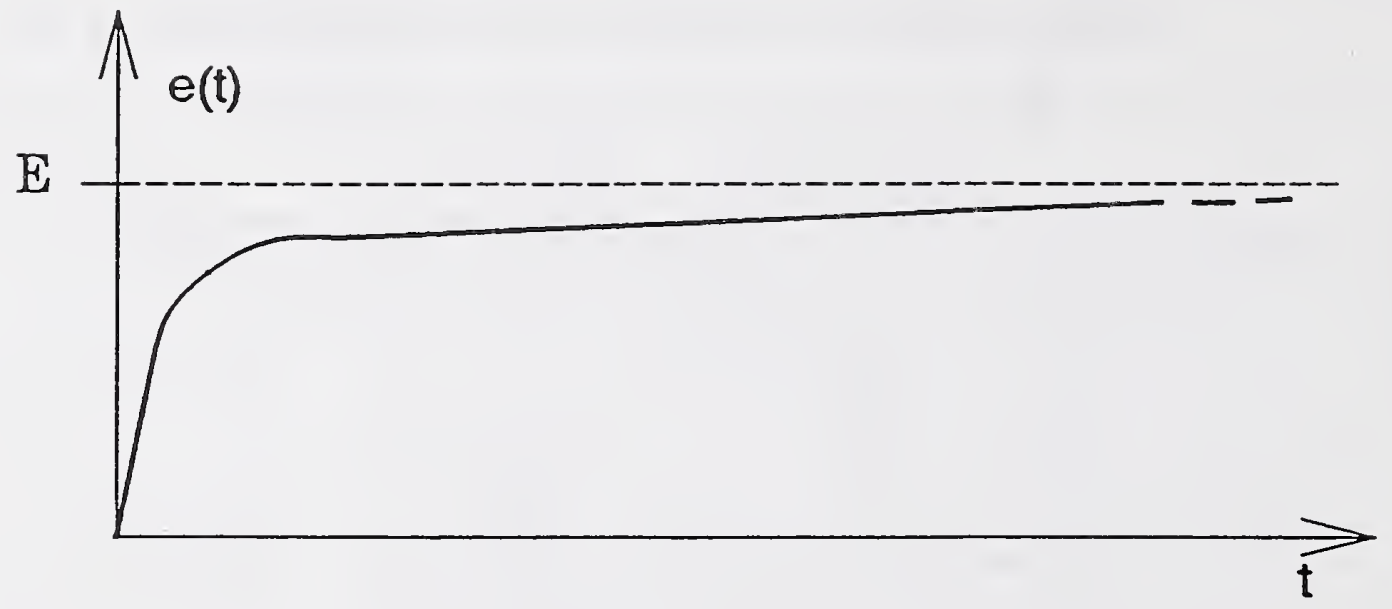

Figure 4.3 Step response of a typical coaxial cable with skin-effect loss. Note the long "dribble-up", that is, the slow approach to the final value, E.

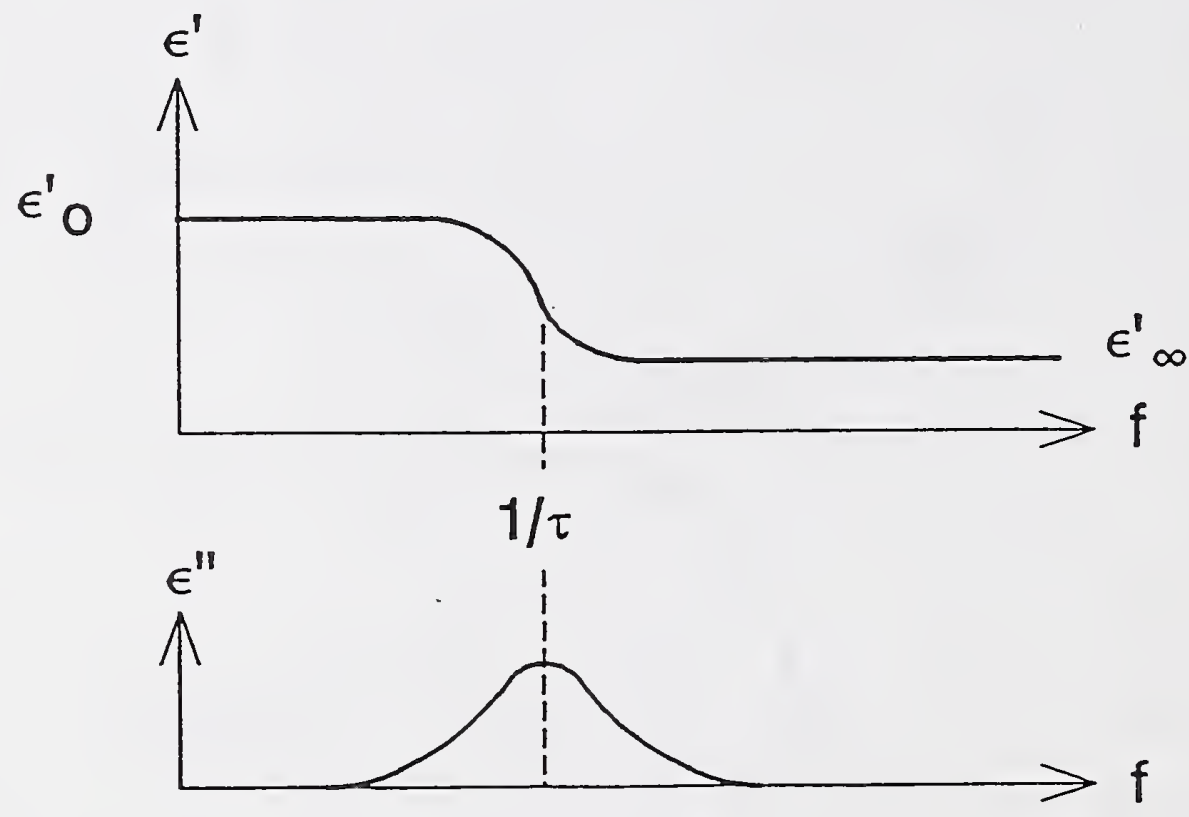

Figure 4.4 Debye-type dielectric function. 


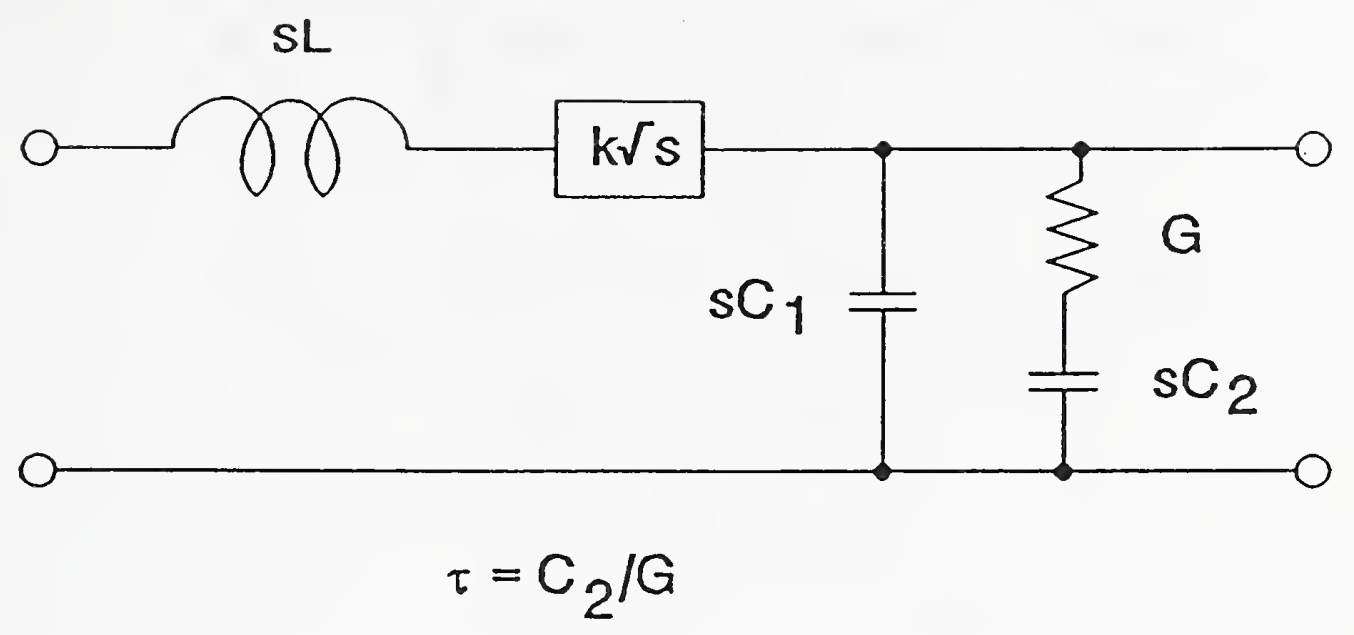

Figure 4.5 Per-unit-length equivalent circuit of a transmission line with Debye-type dielectric.

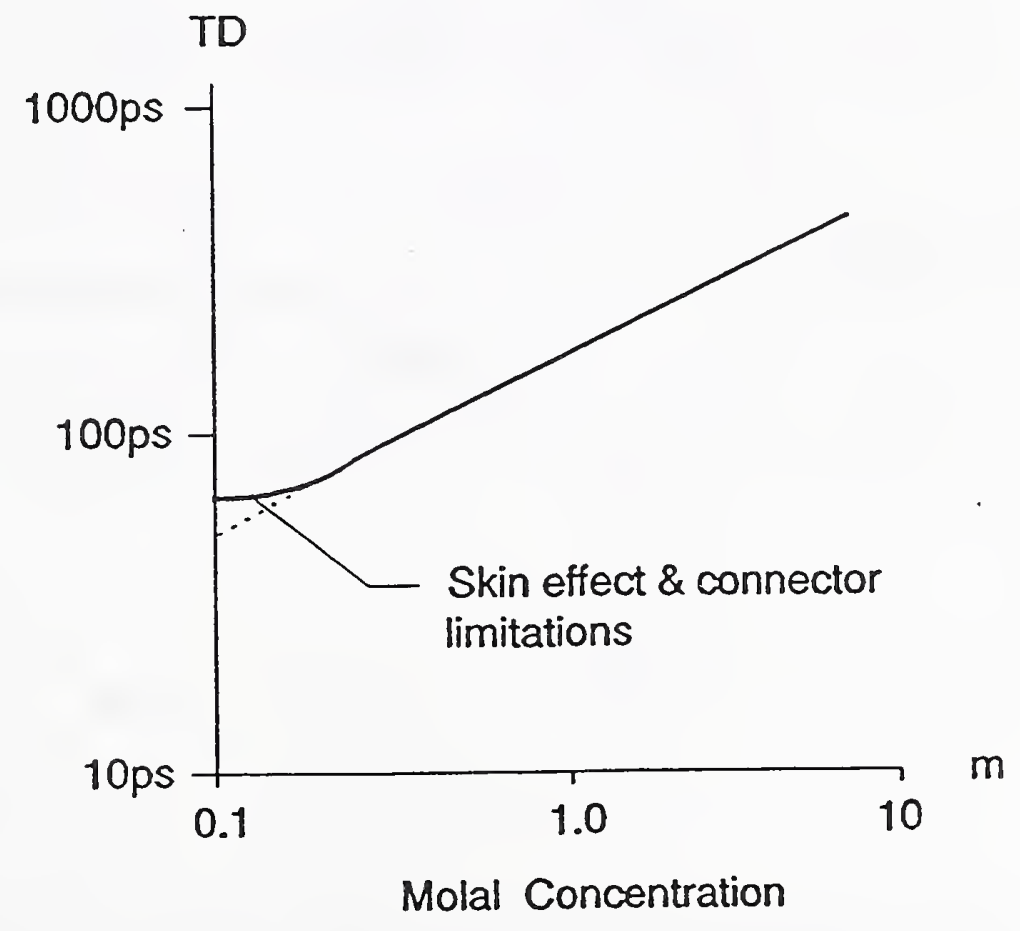

Figure 4.6 Step-response transition duration versus Debye-dielectric doping. 


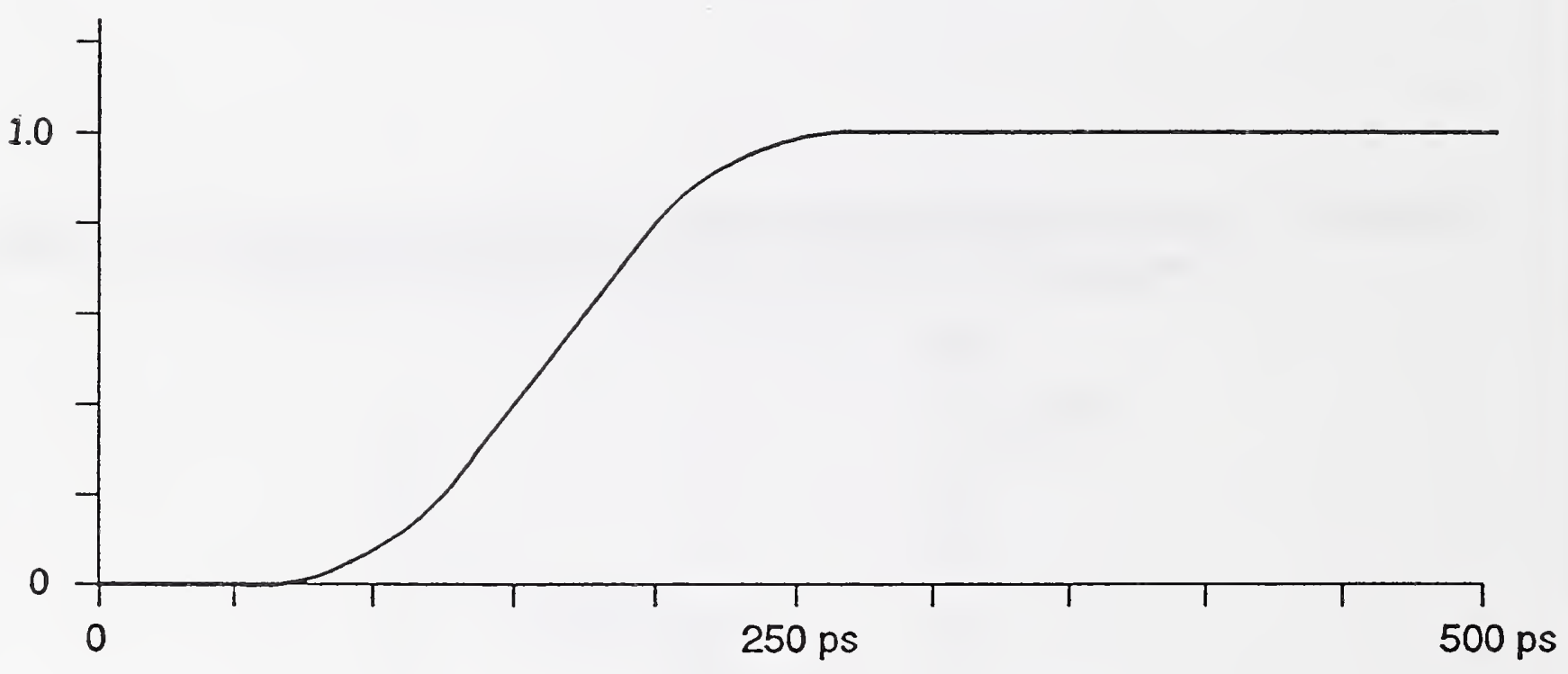

Figure 4.7 Unit-step response of a Debye-dielectric, coaxial low-pass filter. 


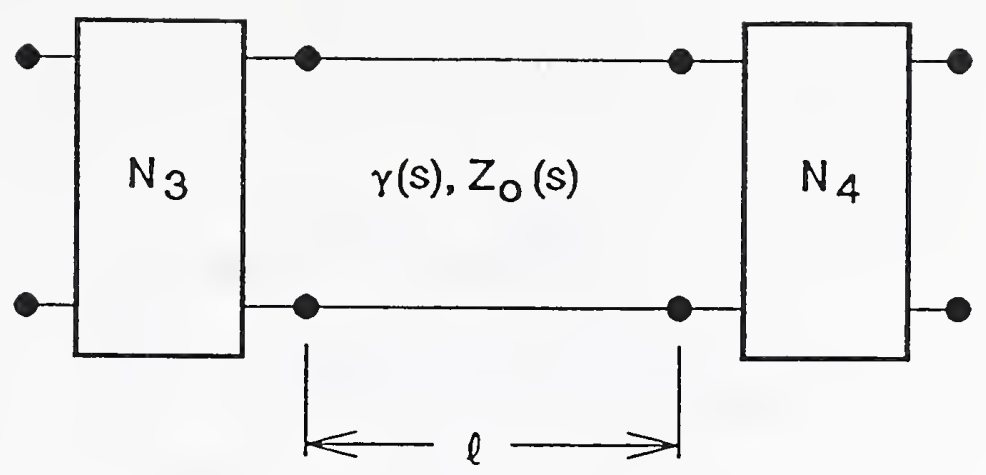

(A)

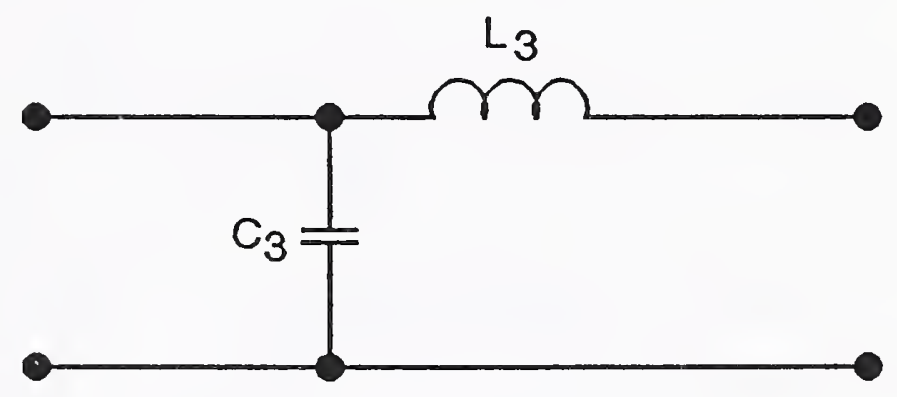

(B)

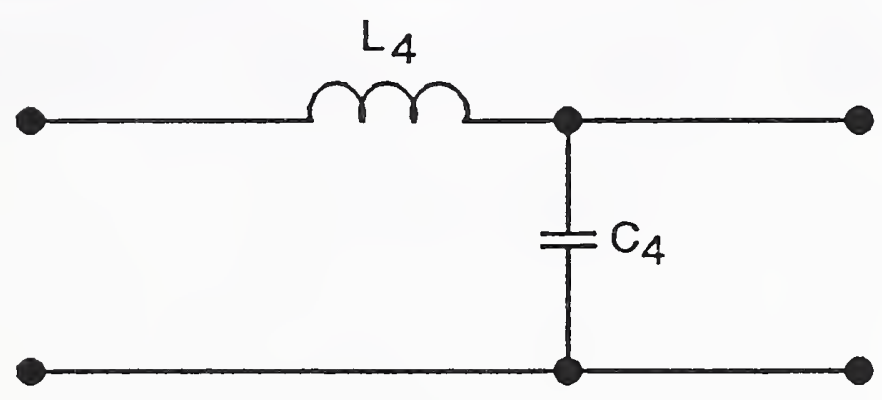

(C)

Figure 4.8 (A) The complete model of the Debye-dielectric filter including the connectors $\mathrm{N}_{3}$ and $\mathrm{N}_{4}$. (B) The equivalent circuit for connector $\mathrm{N}_{3}$. (C) The equivalent circuit for connector $\mathrm{N}_{4}$. 


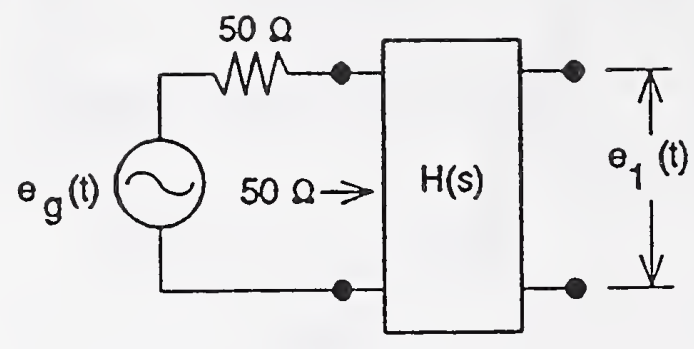

(A)

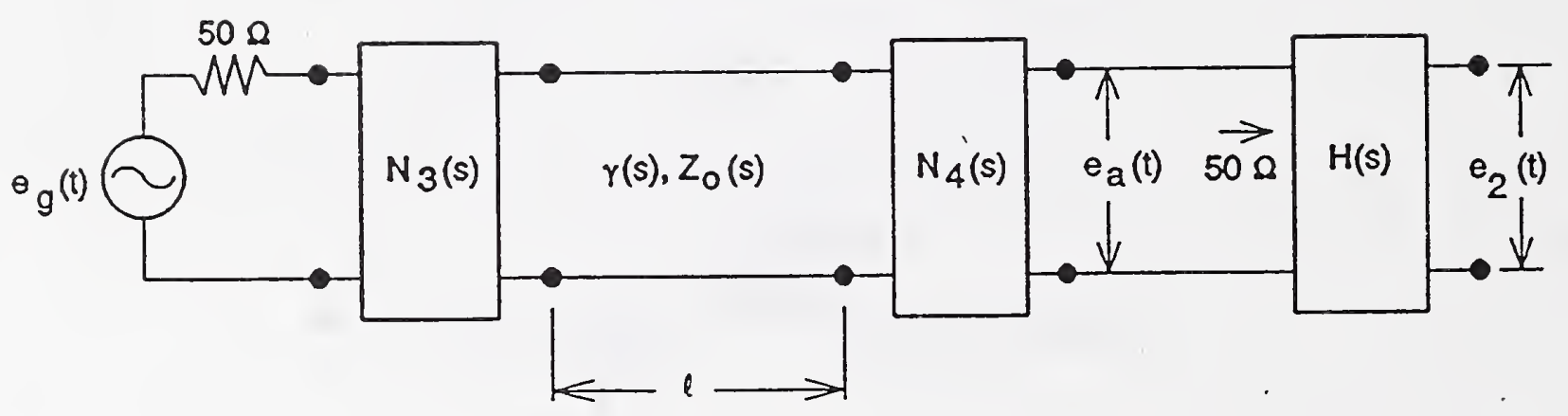

(B)

Figure 4.9 (A) The tunnel-diode transition generator operating into a $50 \Omega$ load with the load voltage being observed by the oscilloscope system, $H(s)$. (B) The tunnel-diode transition generator driving the low-pass filter with the low-pass filter load voltage being observed by the oscilloscope system, $H(s)$. 


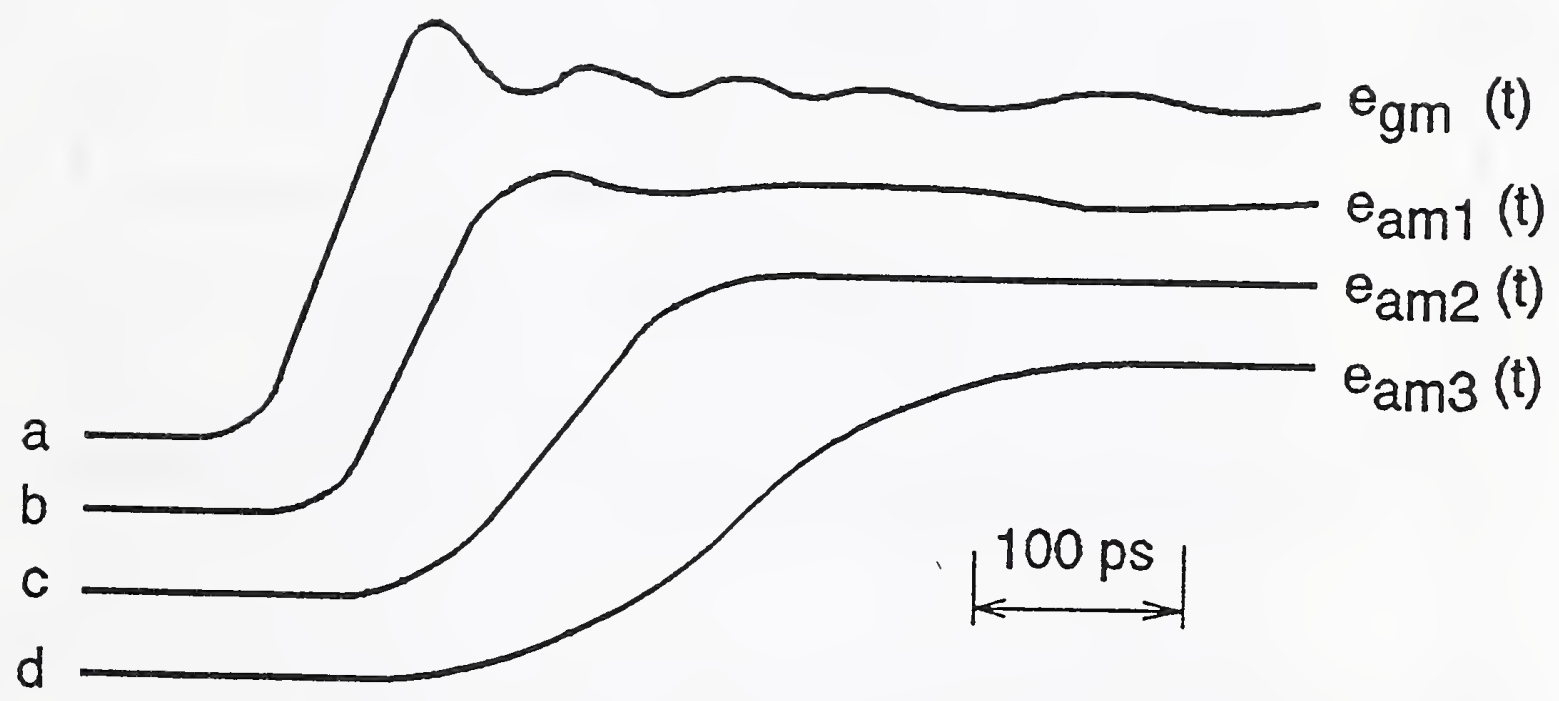

Figure 4.10 Measured and deconvolved waveforms from the (a) tunnel diode and the (b) 50 ps, (c) 100 ps, and (d) 200 ps low-pass filters. 


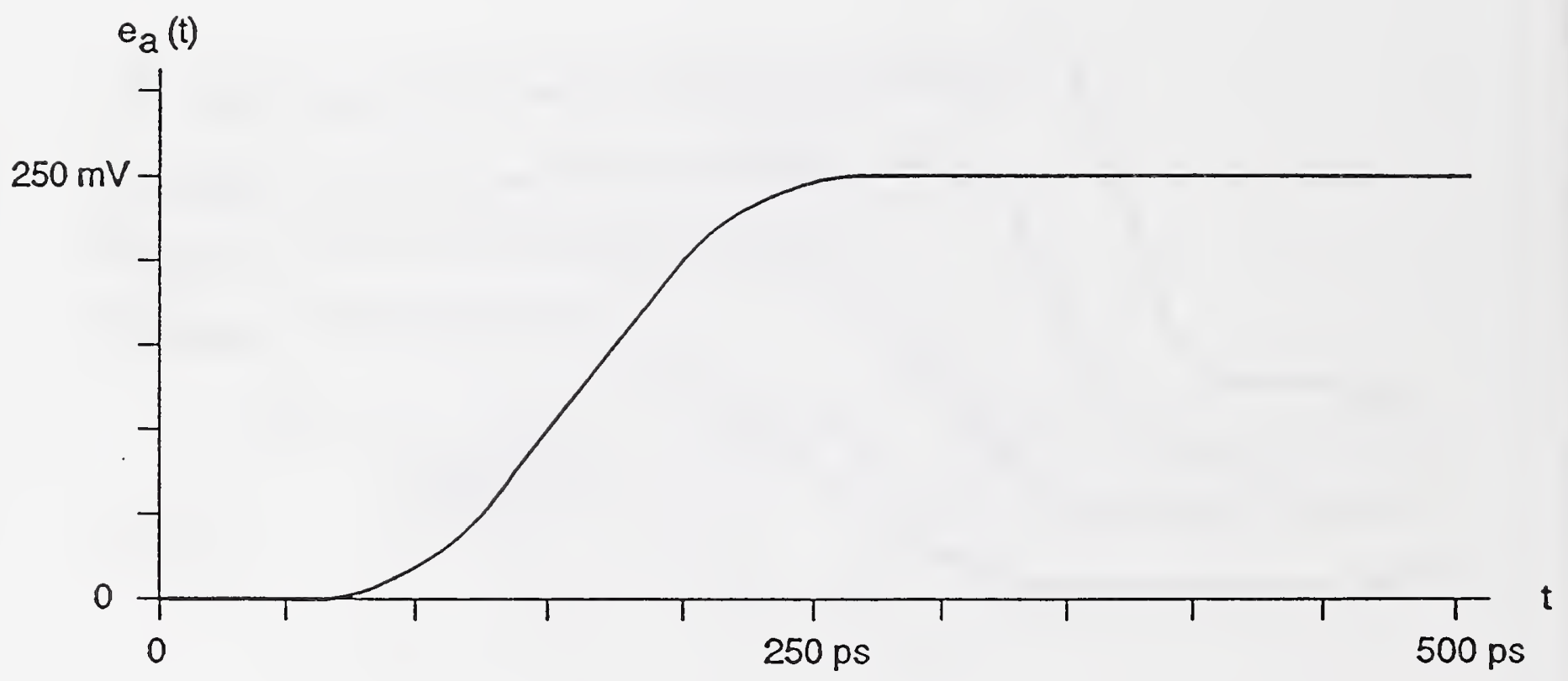

Figure 4.11 Available reference pulse into a $50 \Omega$ load from the TD/100 ps filter combination, $e_{\mathrm{a}}(t)$. 


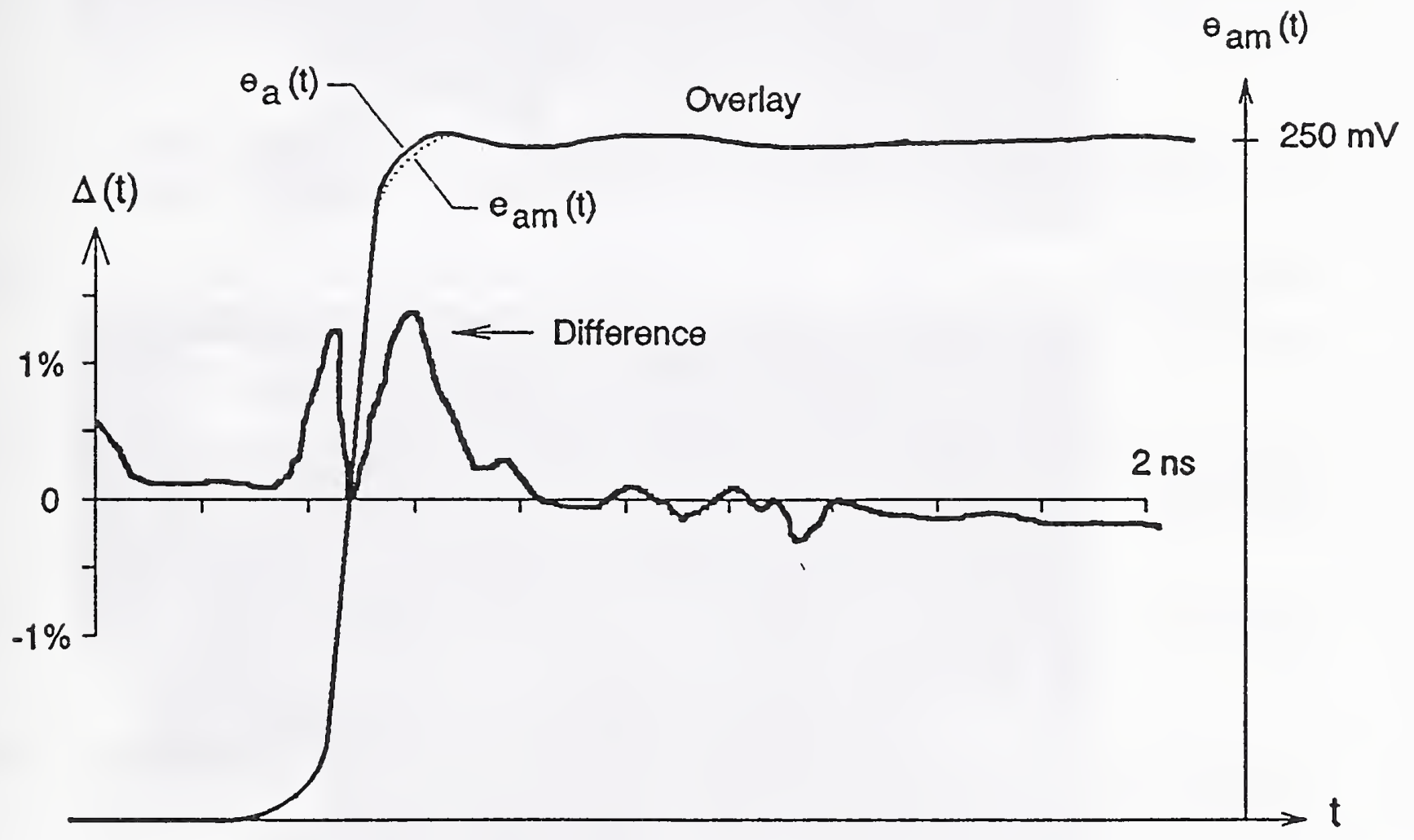

Figure 4.12 Overlay of a predicted 100 ps reference pulse, $e_{a}(t)$, the actual measured waveform (dotted line), $e_{\text {am }}(t)$, and their resultant difference, $\Delta(t)$. The waveforms are aligned so that their $50 \%$ values coincide with each other. 


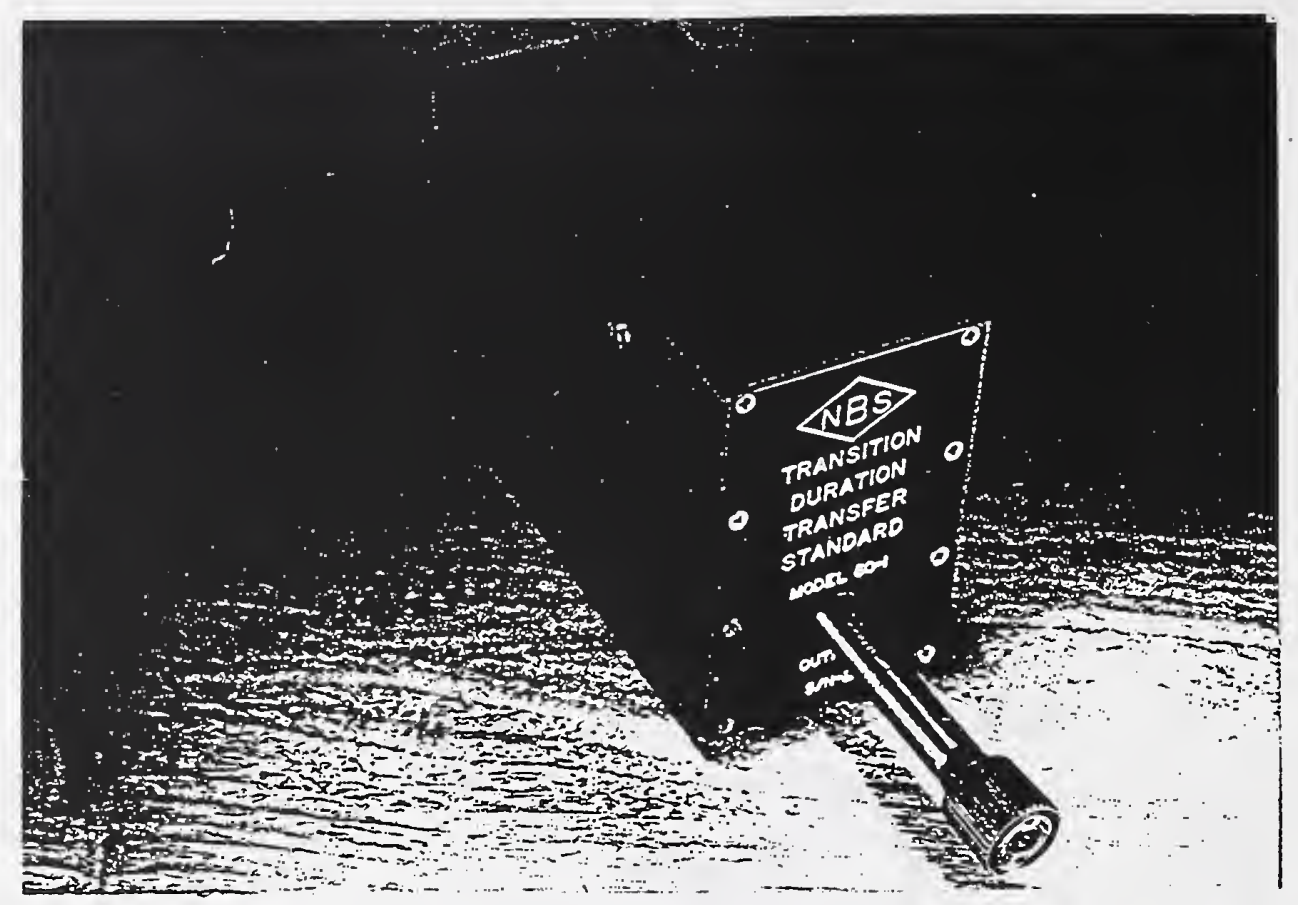

(A)

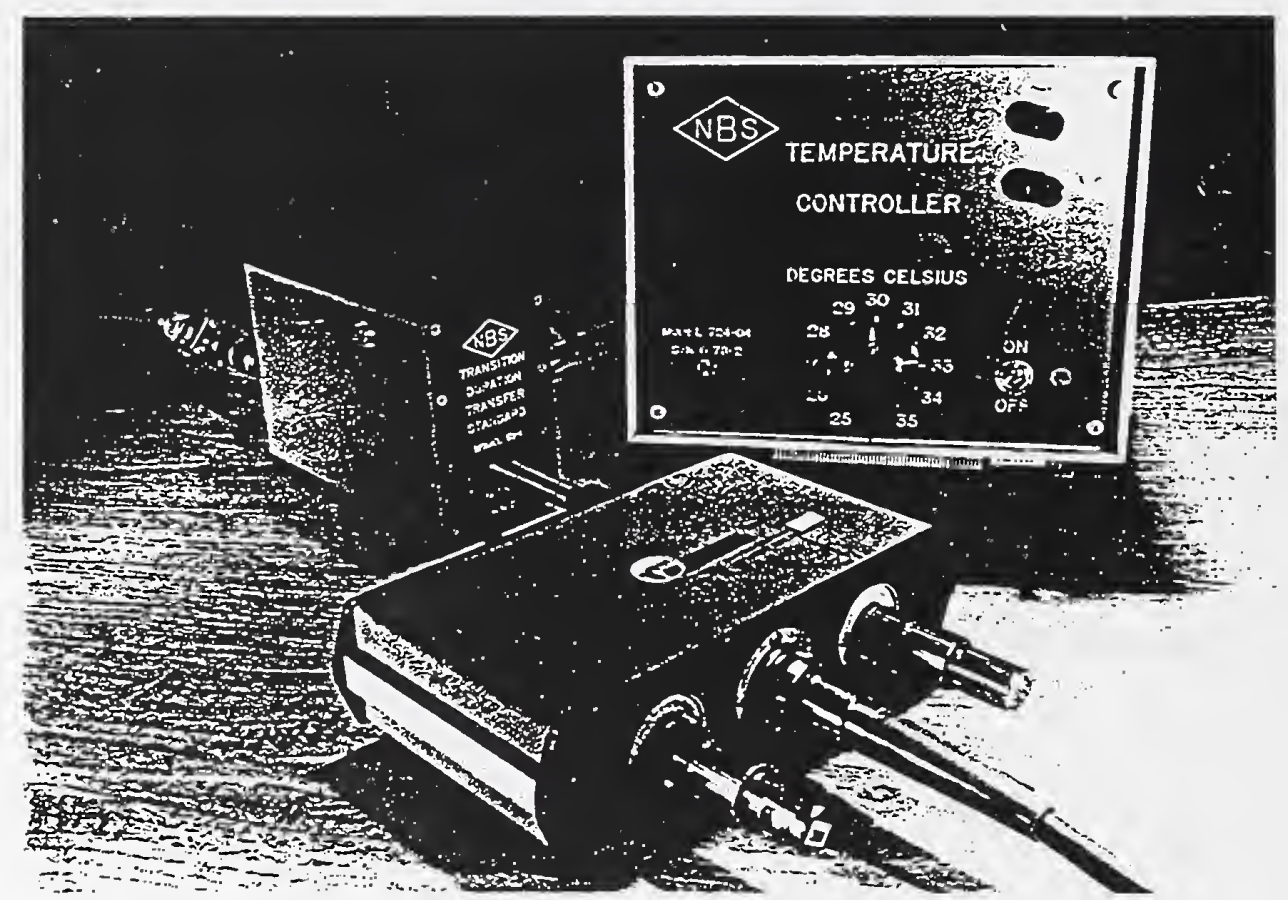

(B)

Figure 4.13 (A) Photograph of the NBS reference waveform pulse generator. (B) Generator shown with associated temperature controller, tunnel-diode pulse generator (attached to rear APC-7 connector), and APMS dual-channel sampler (attached to front APC-7 connector). 


\section{References}

[1] "IRE Standards on Pulses: Methods of Measurement of Pulse Quantities, 1955", Proc. IRE, Vol. 43, No. 11, pp. 1610-1616, Nov. 1955, also IEEE Std. 181-1955.

[2] "Standards on Pulses: Definitions of Terms - Part I, 1951," Proc. IRE, Vol. 39, No. 6, pp. 624-626, June 1951 Part II, 1952", Proc. IRE, Vol. 40, No. 5, pp. 552-554, May 1952, also IEEE Std. 194-1952.

[3] "IEEE Pulse Measurement and Analysis, General Considerations", IEEE Standard 181-1977, The Institute of Electrical and Electronic Engineers, N.Y., N.Y., July 22, 1977.

[4] "IEEE Glossary of Pulse Terms and Definitions", IEEE Standard 194-1977, The Institute of Electrical and Electronics Engineers, N.Y., N.Y., July 26, 1977.

[5] "IEC, Pulse Techniques and Apparatus, Part I: Pulse Terms and Definitions", Publication No. 469-1, First Ed., International Electrotechnical Commission, Geneva, Switzerland, 1974.

[6] "IEC, Pulse Techniques and Apparatus, Part II: Pulse Measurement and Analysis General Considerations", Publication No. 469-2, First Ed., 1974, International Electrotechnical Commission, Geneva, Switzerland, 1974.

[7] Nahman, N.S.; et al. "Applications of time domain methods to microwave measurements." IEE Proc. 127, Pt. H, (2), pp. 100-106, April 1980.

[8] Gans, W. L.; et al. "Application of an automated pulse measurement system to telecommunication measurements." Proc. URSI Symposium on Measurements in Telecommunications, Lannion, France, 165-170; 1977 October. Published by la Centre National D'Etudes des Telecommunications, Route de Tregastel, 22301 Lannion, France.

[9] Gans, W. L.; "Present capabilities of the NBS automatic pulse measurement system." IEEE Trans. Inst. \& Meas., Vol. IM25(4), pp. 384-388, December 1976. 
[10] Gans, W. L.; "A time domain automatic network analyzer for microwave measurements," Master of Science Degree Thesis, Electrical Engineering Dept., University of Colorado, Boulder, Colorado, 1975.

[11] Gans, W. L.; Andrews, J. R.; "Time domain automatic network analyzer for measurement of RF and microwave components." Technical Note 672, National Bureau of Standards, Boulder, Co. 80303, July, 1975.

[12] Riad, S. M.; "The Theory and Application of the Homomorphic Transformation to Time Domain Spectroscopy and Scattering Problems," Chapter VI, Ph.D. Dissertation, University of Toledo, Toledo, Ohio, August 1976. (The experimental work was performed at NIST, Boulder, Colorado, by the author as a Guest Worker in the Time Domain Metrology Group, 724.04.)

[13] Andrews, J. R.; Gans, W. L.; "Time domain automatic network analyzer." L'Onde Electrique, Paris, France, 55(10) pp. 569-574, October 1975.

[14] Andrews, J. R.; "Automatic network measurements in the time domain." Proc. IEEE, 66(4), pp. 414-423, April 1978.

[15] McGillem, C. D.; Cooper, G. R.; "Continuous and discrete signal and system analysis." Holt Rinehart, and Winston, Inc., New York, 1974.

[16] Nahman, N. S.; Guillaume, M. E.; "Deconvolution of time domain waveforms in the presence of noise." Technical Note 1047, National Bureau of Standards, Boulder, Co. 80303, 115 pages, October 1981.

[17] Guillaume, M. E.; Bizeul, J.; "Impulse response of a transmission medium: application to optical fibers," Annales Des Telecommunications, Vol. 36, No. 3-4, pp. 1/8-8/8, March-April 1981.

[18] Op. Cit., [15], page 178 and Figure 5-67.

[19] Brigham, E. O.; "The fast Fourier transform," New Jersey: Prentice-Hall, 1974.

[20] Gans, W. L.; "The deconvolution of time jitter in equivalent-time waveform samplers," Digest of Papers, p. G-6, Conference on Precision Electromagnetic Measurements, June 28-July 1, 1982, Boulder, Co. (IEEE Catalog No. 82CH1737$6)$. 
[21] Andrews, J. R.; Baldwin, E. E.; "Amplitude-calibrator for oscilloscopes calibration," NBSIR 81-1646, National Bureau of Standards, Boulder, Co. 80303, 26 pages, April 1981.

[22] Andrews, J. R.; Gans, W. L.; "Pulsed wavemeter timing reference for sampling oscilloscope calibration," IEEE Trans. Inst. \& Meas. Vol. IM24(1), p. 82, March 1975.

[23] Riad, S. M.; Nahman, N. S.; "Modeling of the feed-through wideband (DC to 12.4 $\mathrm{GHz}$ ) sampling head," Digest of the 1978 IEEE-MTT-S International Microwave Symposium, Ottawa, Canada, June 27-29, 1978.

[24] Riad, S. M.; "Modeling of the HP-1430A feedthrough wideband (28 ps) sampling head," IEEE Trans. Inst. \& Meas., Vol. IM-31(2), pp. 110-115, June 1982.

[25] Nahman, N. S.; "The measurement of baseband pulse rise times of less than 1 nanosecond," Proc. IEEE, Vol. 55, No. 6, pp. 855-864, June 1967, see section VI.

[26] Op. Cit., [7], see section 3.

[27] Andrews, J. R.; "Precision picosecond-pulse measurements using a high quality superconducting delay line," IEEE Trans. Inst. \& Meas., Vol. IM-23(4), pp. 468-472, Dec. 1974.

[28] Nahman, N. S.; "Software correction of measured pulse data," Fast Electrical and Optical Measurements, Volume I, pp. 351-417, NATO ASI Series, 1986 Martinus Nijhoff Publishers, Dordrecht, The Netherlands, 1986. USA Library of Congress ISBN 90-2473298-8.

[29] Valley, G. E., Jr.; Wallman, Henry; "Vacuum tube amplifiers," Radiation Laboratory Series, Vol. 18, pp. 77-82, McGraw-Hill, New York, 1948.

[30] Thornton, R. D.; et al., "Multistage transistor circuits," SEEC Series, Vol. 5, John Wiley \& Sons, Inc., New York, 1965, Chap. 8.

[31] Blinchikoff, H. J.; Zverev, A. I.; "Filtering in the time and frequency domains," J. Wiley and Sons, New York, 1976. 
[32] McCaa, Jr., W. D.; Nahman, N. S.; "Generation of reference waveforms by uniform lossy transmission lines," IEEE Trans. Inst. and Meas., Vol. IM-19(4), pp. 382-390, Nov. 1970.

[33] Nahman, N. S.; Jickling, R.; Holt, D. R.; "Reference waveform generation using Debye dielectric dispersion," NBSIR 73-304, National Bureau of Standards, Boulder, Co. 80303, 1972.

[34] Debye, P.; "Polar molecules," Chemical Catalog Co., 1929, also Dover Publications, New York.

[35] Bagozzi, R. P.,;Ives, W. R.; Nahman, N. S.; "Determination of the dielectric relaxation time in a Debye binary liquid by pulse measurements," Progress in Radio Science, 1966-69, Vol. 2, pp. 257-265, URSI, Brussels, 1971.

[36] Andrews, J. R., op. cit. [14].

[37] Nahman, N. S.; "Error criteria and the use of reference waveforms," Proc. of the ATE Seminar/Exhibit, Pasadena, CA, Benwill Publications Corp., Boston, MA 02215, pp. IV-21 to IV-28, Jan 1981.

[38] Parruck, B.; Riad, S. M.; "An optimization criterion for iterative deconvolution," IEEE Trans. Inst. and Meas., Vol. IM-32(1), pp. 137-140, March 1970 
6 Appendix 1:

NBS Pulse Transition Duration Transfer Standard

Construction Drawings 


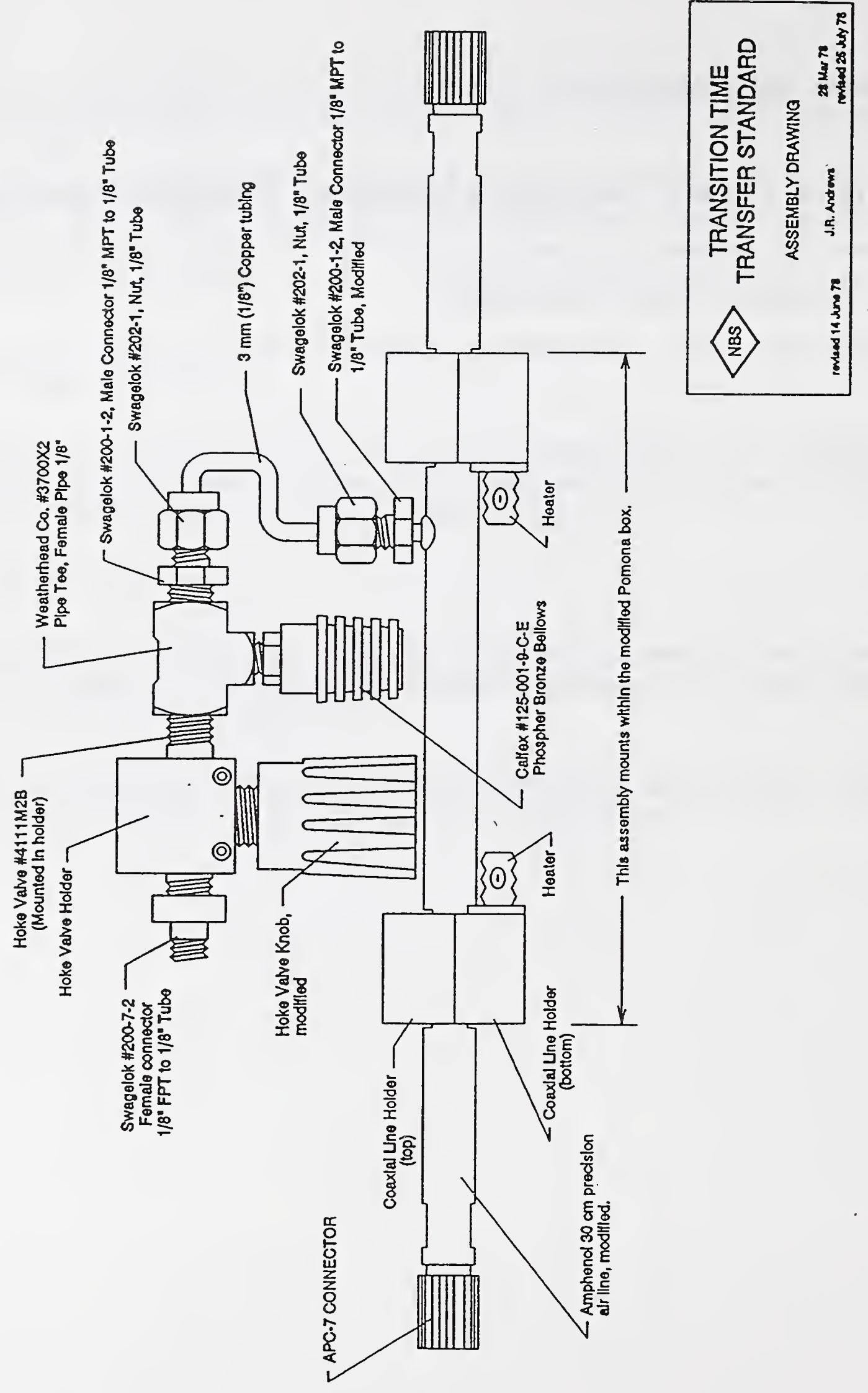




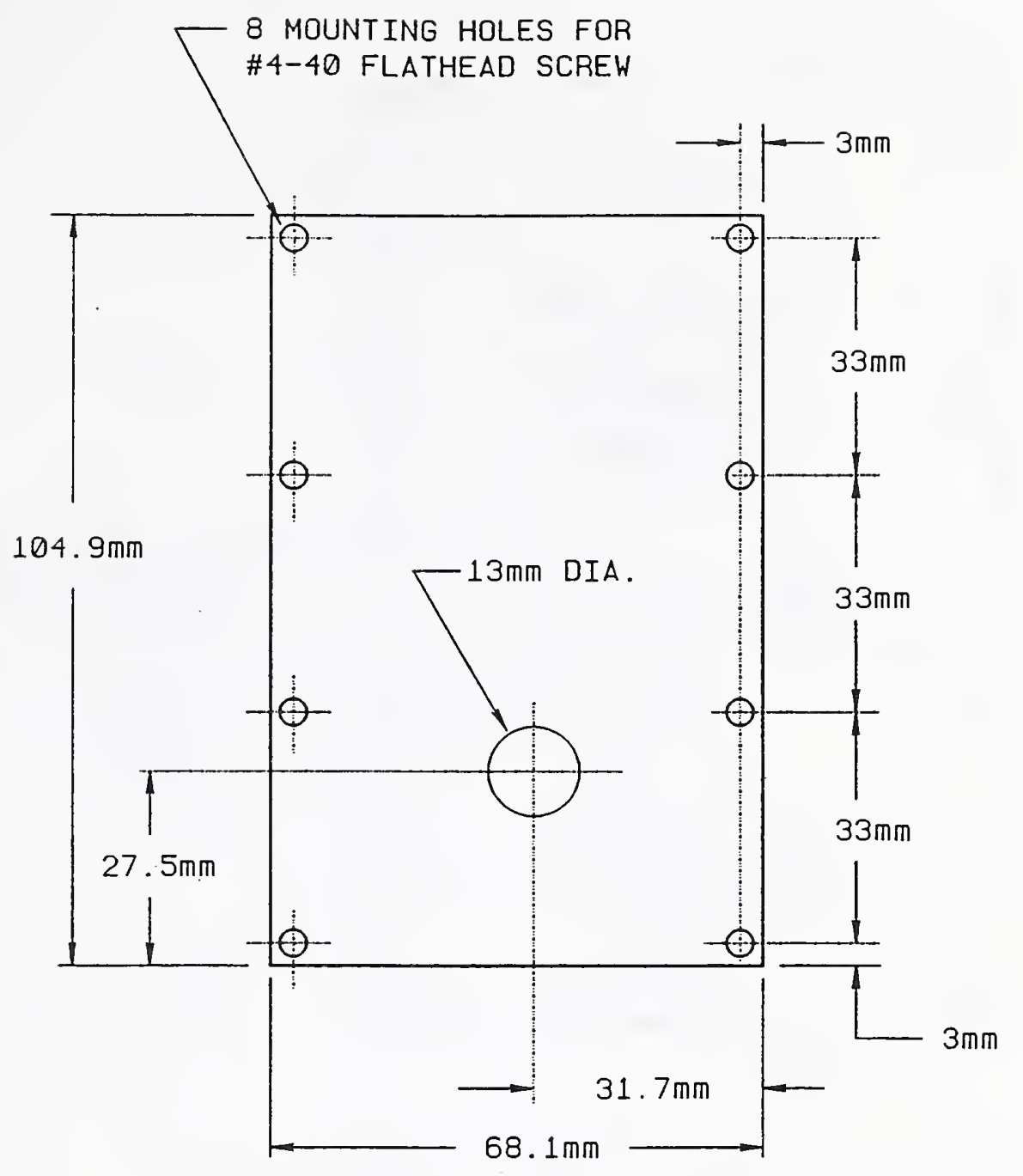

MATERIAL: $2.03 \mathrm{~mm}(0.080 "$ ) THICK ALUMINUM

FINISH: BLUE ANODIZE-COLOR SIMILAR TO POMONA BOX \#3742

ENGRAVING: SEE PAGE 2

QUANTITY: 1 REQUIRED

OUTPUT END PLATE

PAGE 1 OF 2

J.R. ANDREWS 14 JUNE 78 


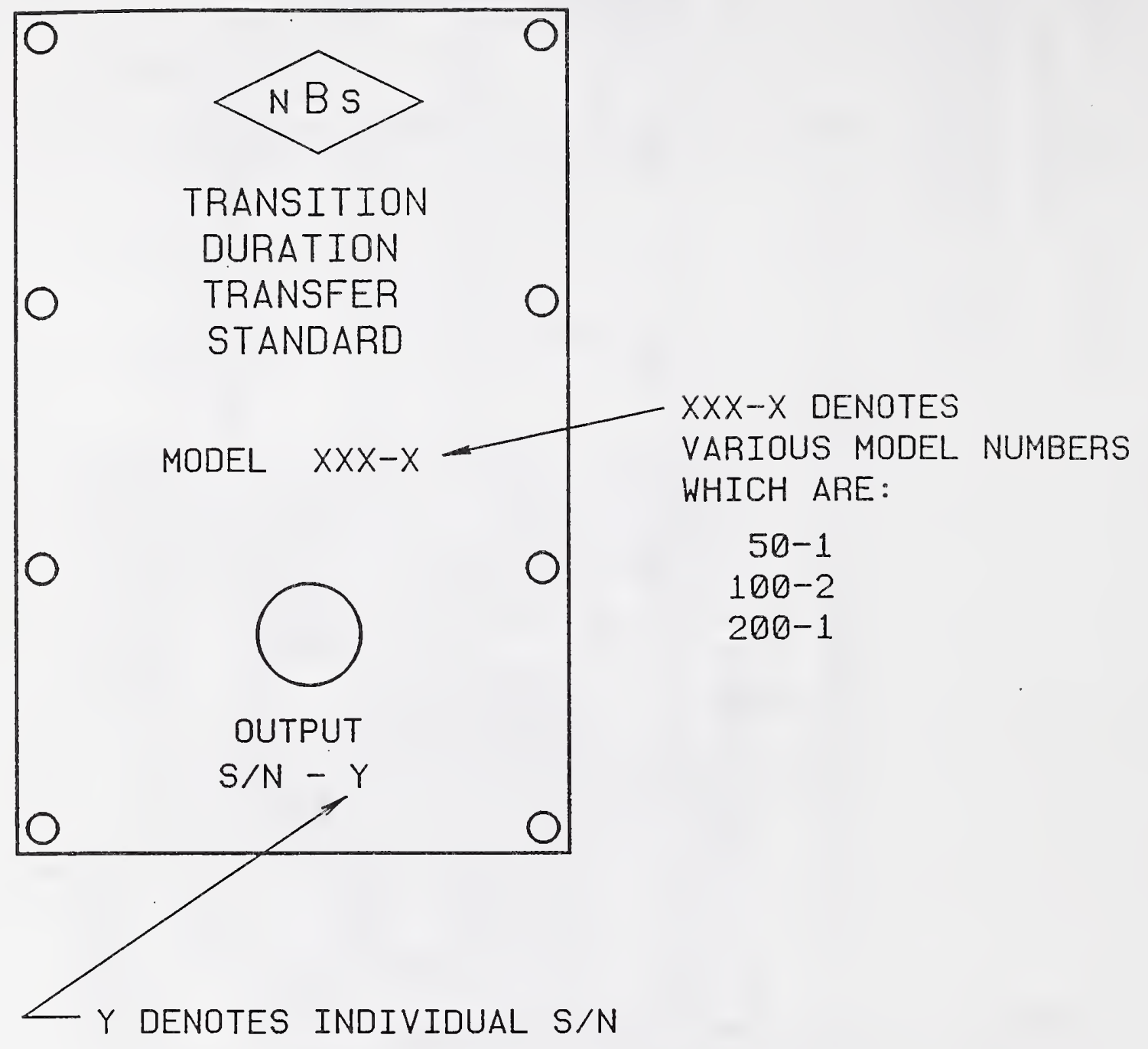

OUTPUT END PLATE

PAGE 2 OF 2

J.R. ANDREWS 14 JUNE 78 


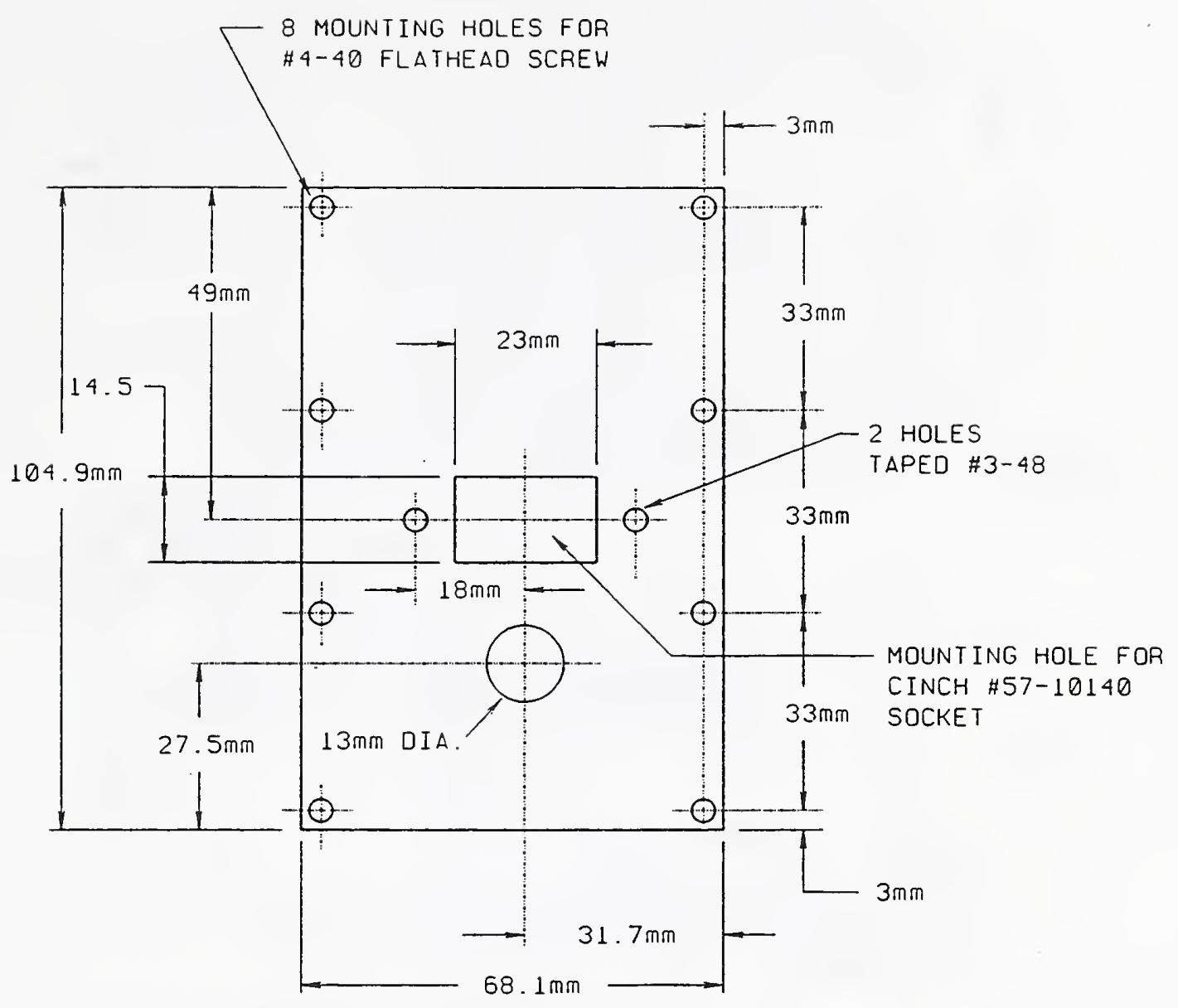

MATERIAL: $2.03 \mathrm{~mm}(0.080 ")$ THICK ALUMINUM

FINISH: BLUE ANODIZE-COLOR SIMILAR TO POMONA BOX \#3742

ENGRAVING: NONE

QUANTITY: 1 REQUIRED

INPUT END PLATE

PAGE 1 OF 1

J.R. ANDREWS 14 JUNE 78 


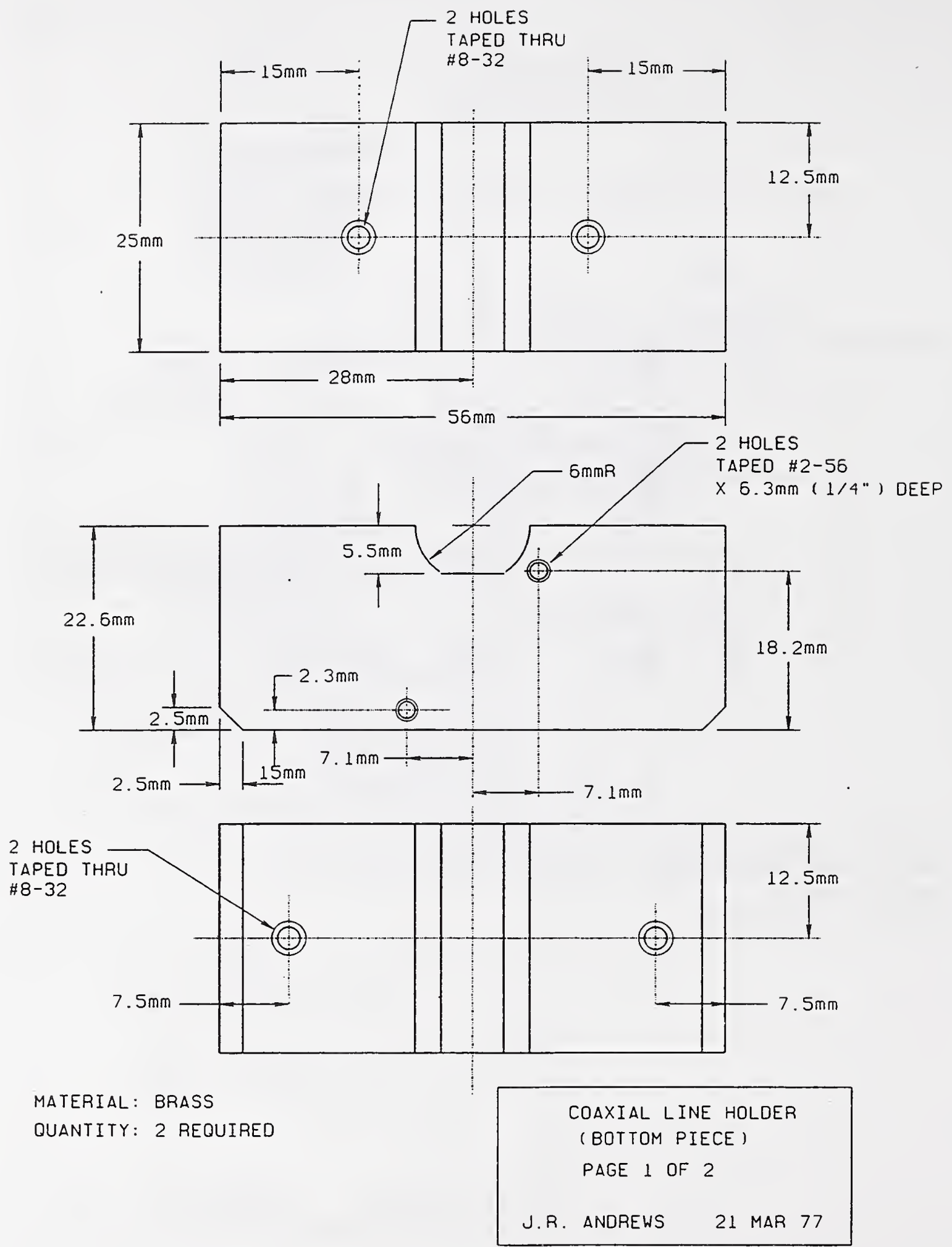



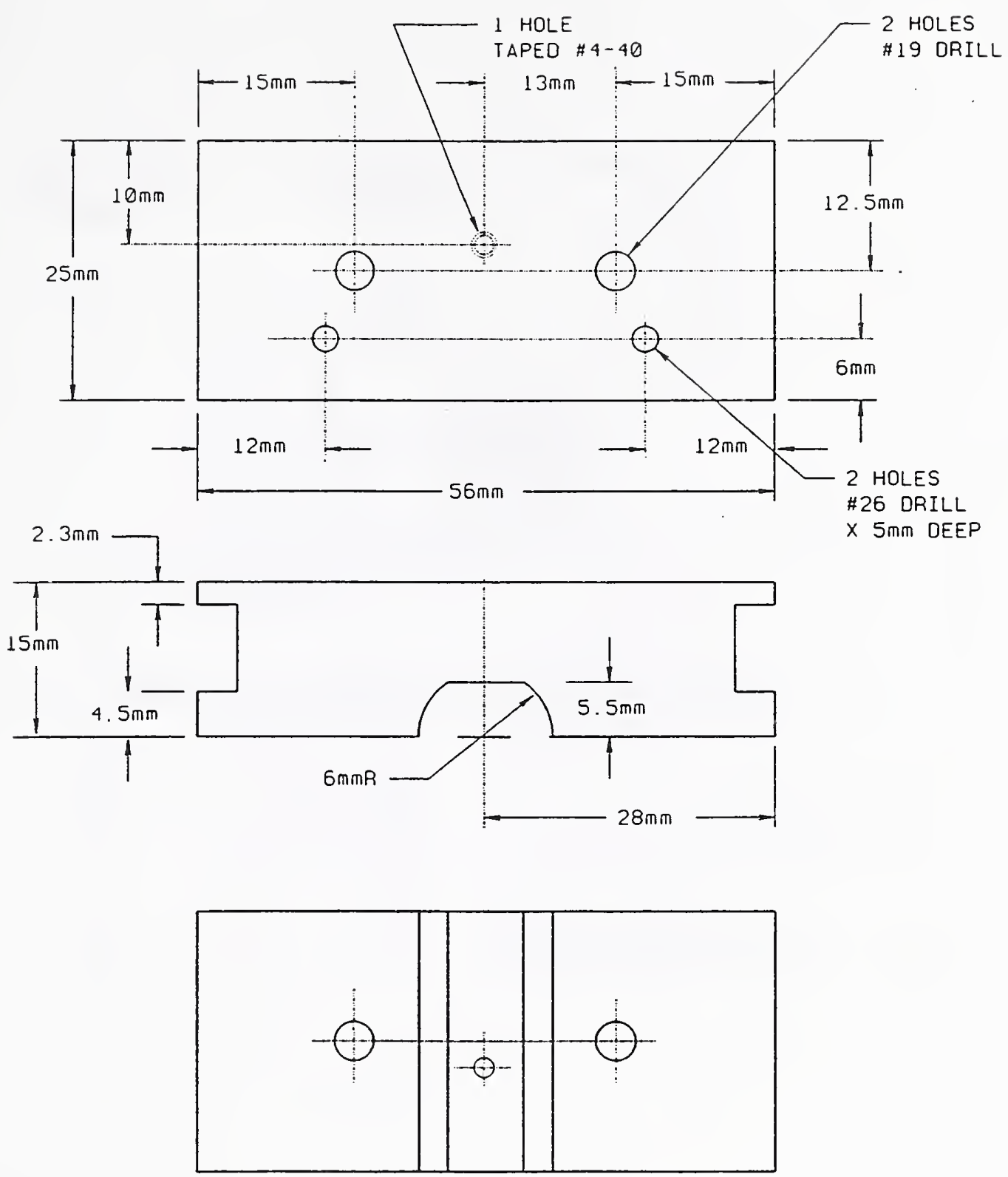

MATERIAL: BRASS

QUANTITY: 2 REOUIRED

\begin{tabular}{|c|}
\hline \\
COAXIAL LINE HOLOER \\
( TOP PIECE) \\
PAGE 2 OF 2 \\
J.R. ANOREWS 21 MAR 77
\end{tabular}




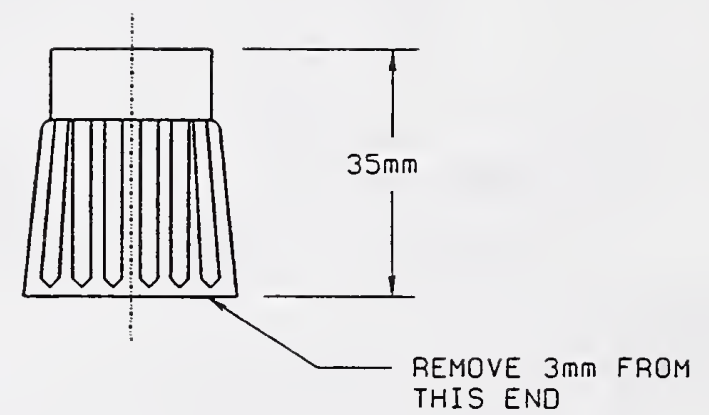

MATERIAL: VALVE HANDLE FROM HOKE \#4111M2B VALVE QUANTITY: 1 REQUIRED

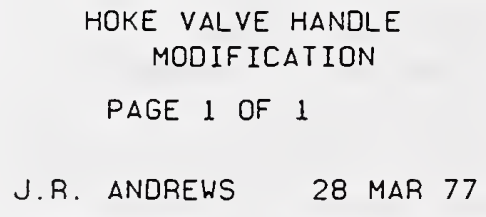




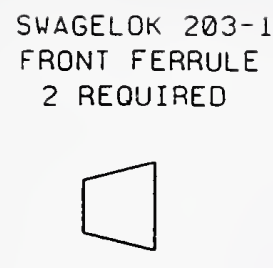

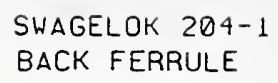

2 REOUIRED

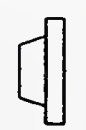

SHAGELOK 202-1

NUT

2 REOUIRED

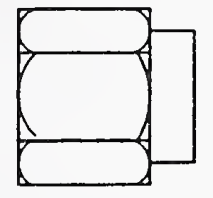

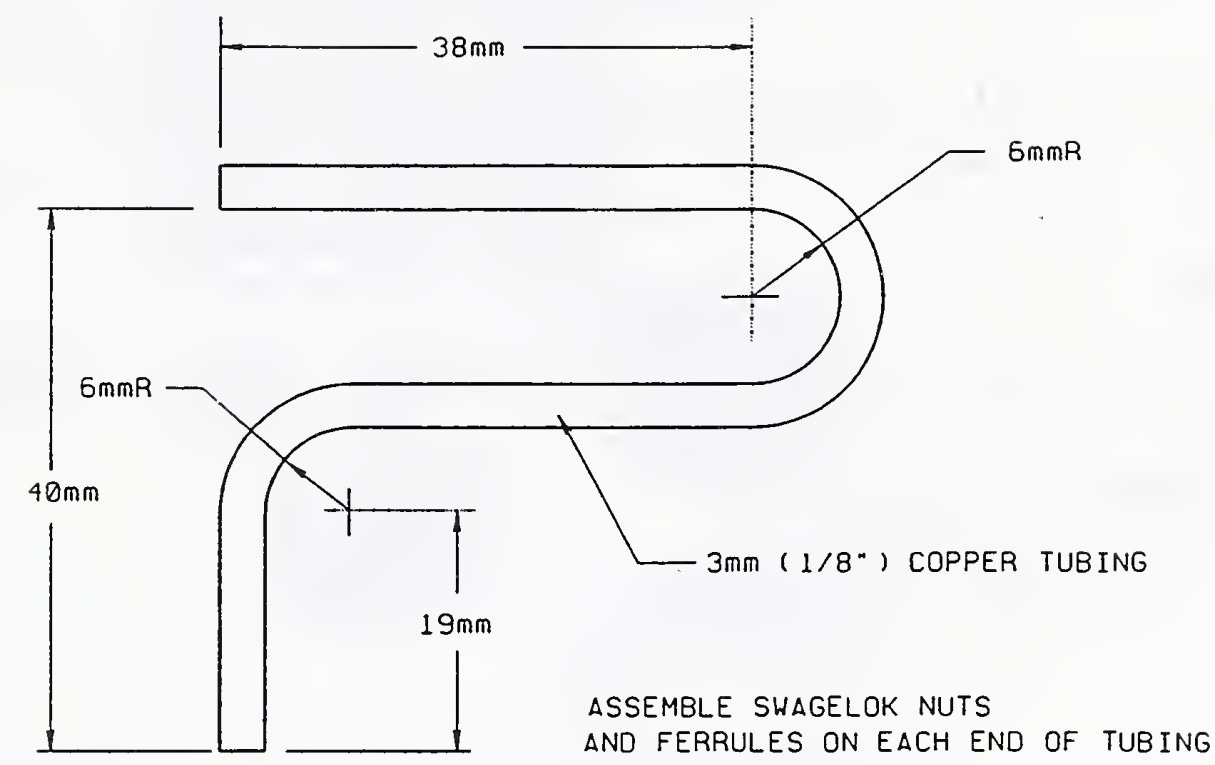

ANO FERRULES ON EACH END OF TUBING

INTERCONNECT TUBING

PAGE 1 OF 1

J.R. ANDREWS 28 MAR 77 


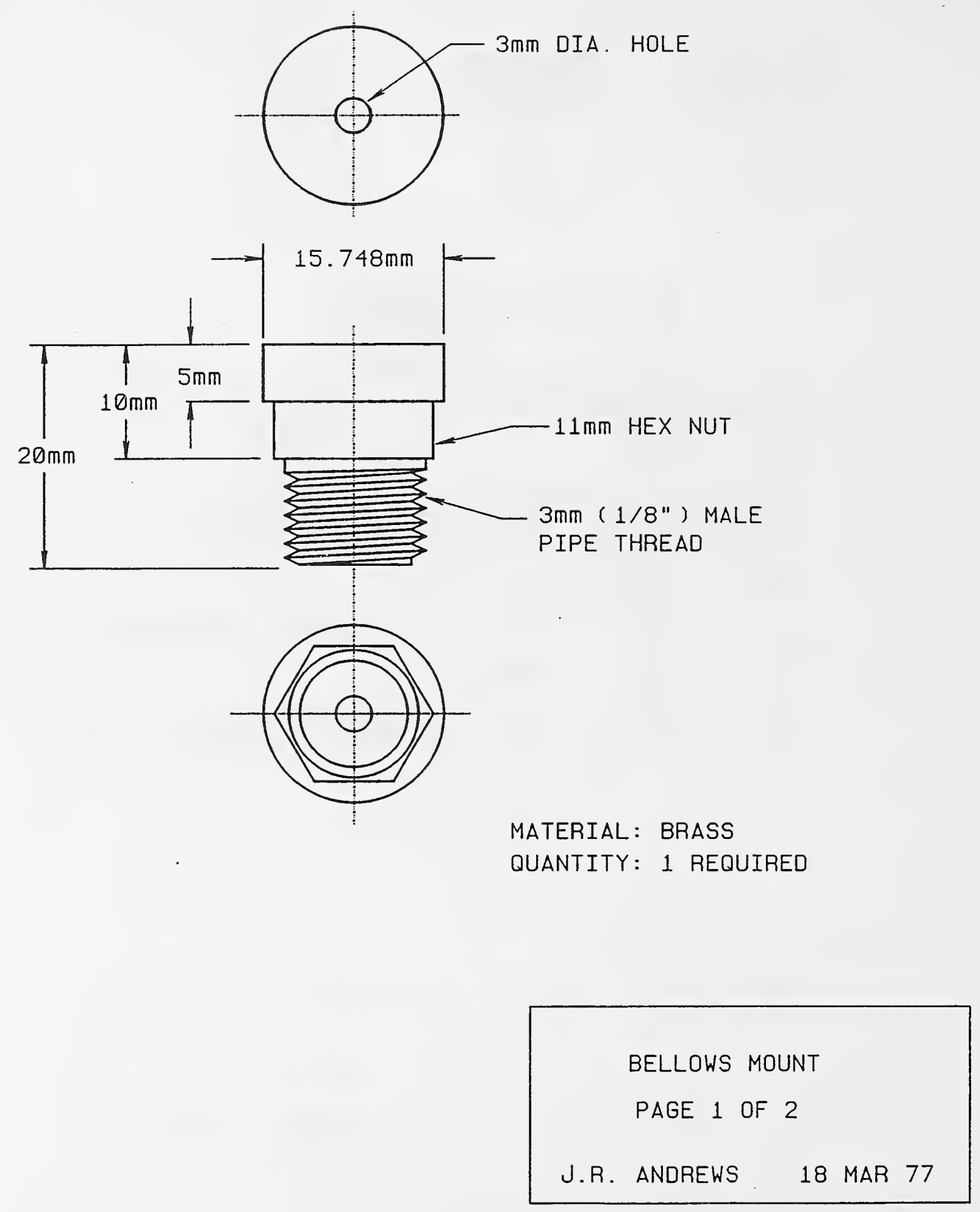


MATERIAL: CALFEX CORP. DETROIT, MI. BELLOWS

CODE \#125-D01 WITH 9 CONVOLUTIONS. TYPE C

CLOSED END. AND TYPE E COLLAR (3mm LONG)

MATERIAL REF : PHOSPHER BRONZE

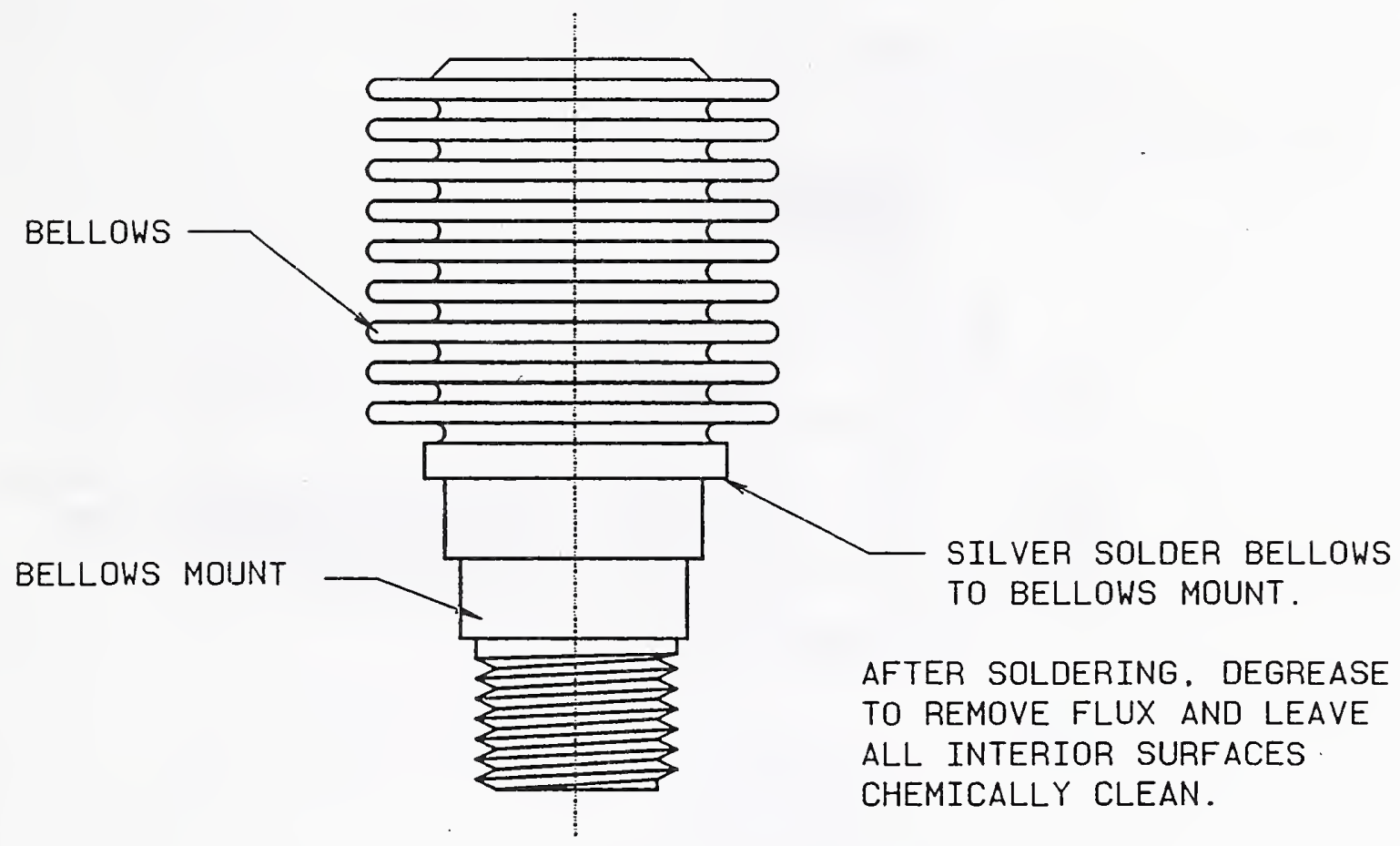

BELLOWS MOUNT

PAGE 2 OF 2

J.R. ANDREWS 18 MAR 77 
MATERIAL: BRASS SWAGELOK MODEL 200-1-2

MALE CONNECTOR. $3 \mathrm{~mm}$ TUBE TO $3 \mathrm{~mm}$ MPT.

QUANTITY: 1 REQUIRED

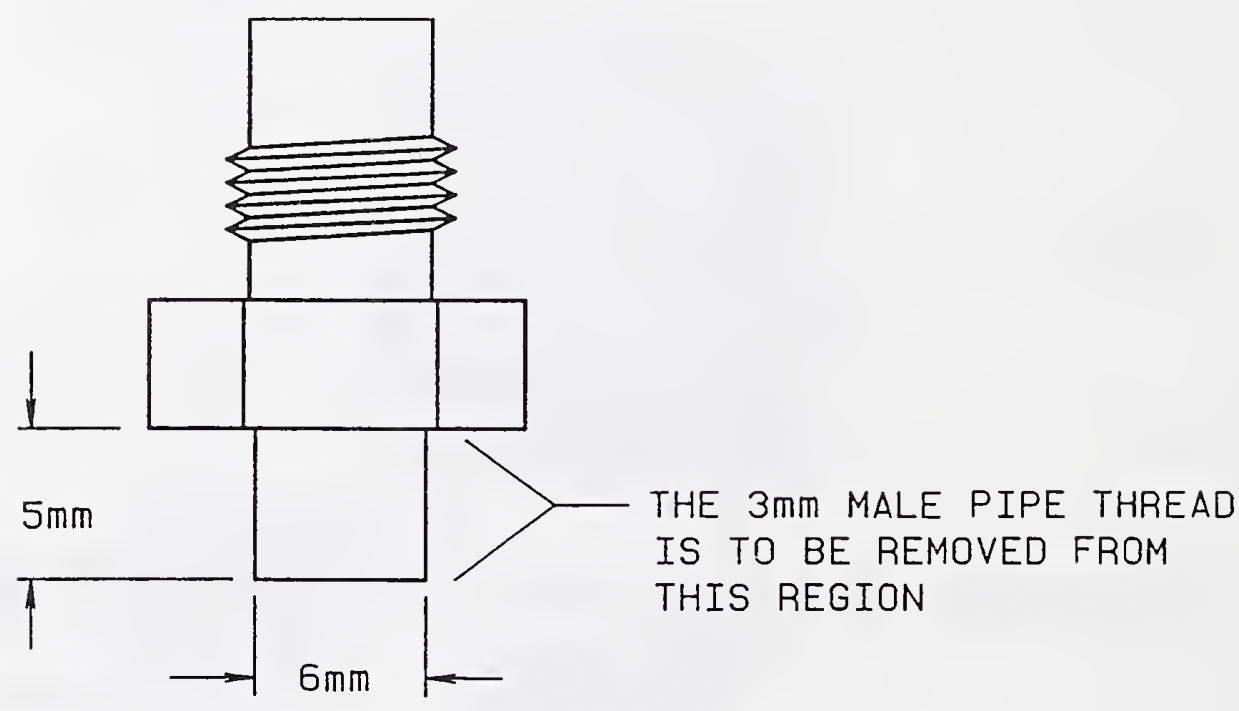

THIS ITEM IS TO BE SILVER SOLDERED TO THE COAXIAL LINE.

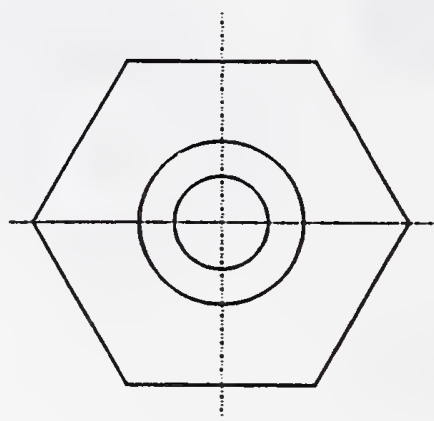
IS TO BE REMOVED FROM THIS REGION 


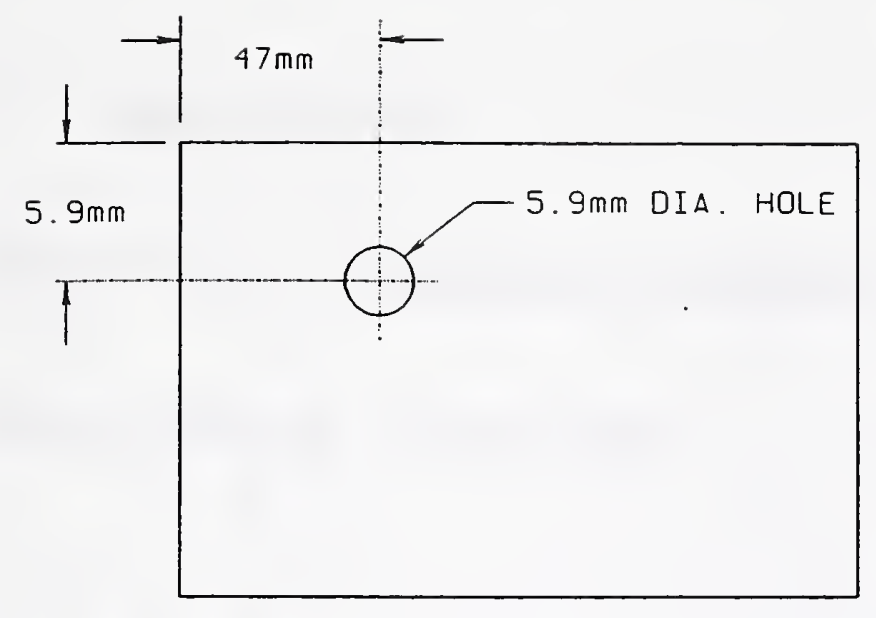

MATERIAL: POMONA MODEL 3742 ALUM. BOX

QUANTITY: 1 REQUIRED

HOLES:

$A=\# 19$ DRILL
$B=$ DRILL AND TAP \#4-40
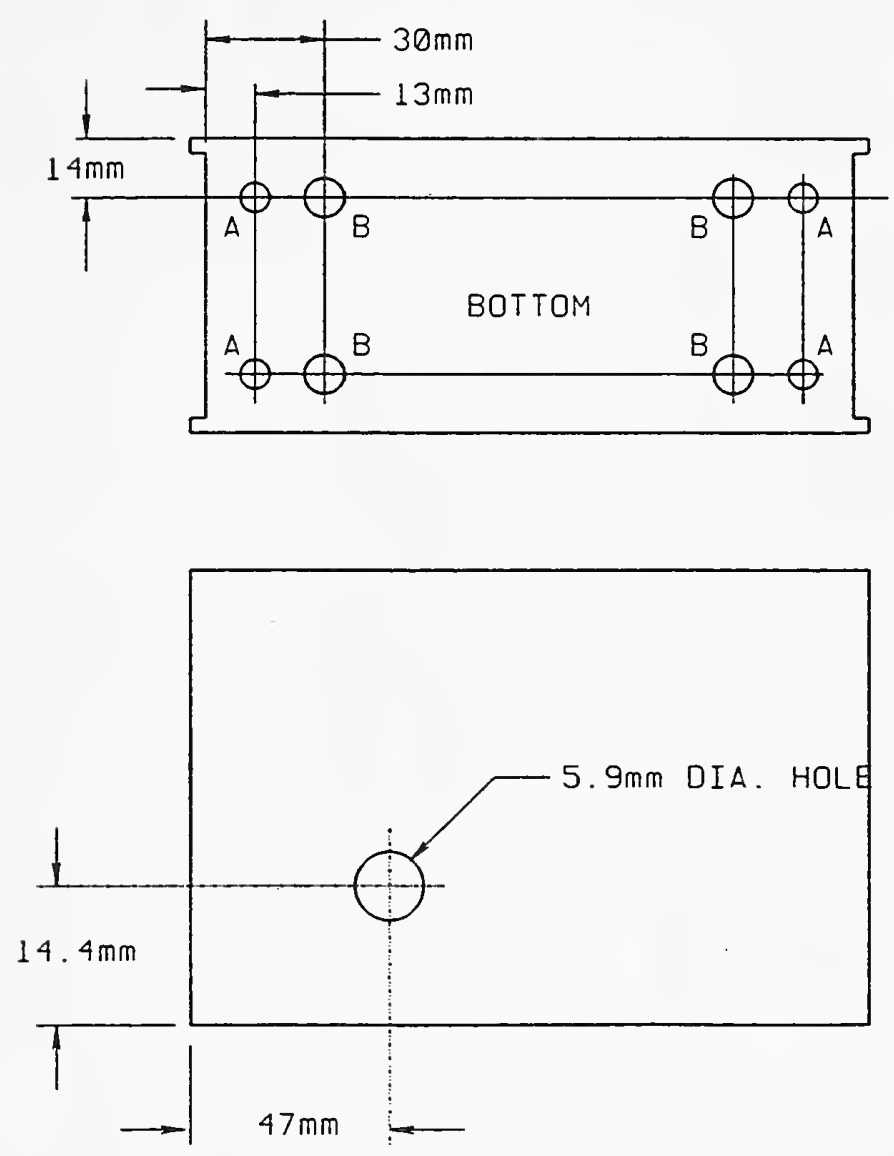

POMONA BOX
MODIFICATION
PAGE 1 OF 1
J.R. ANDREWS 25 MAR 77



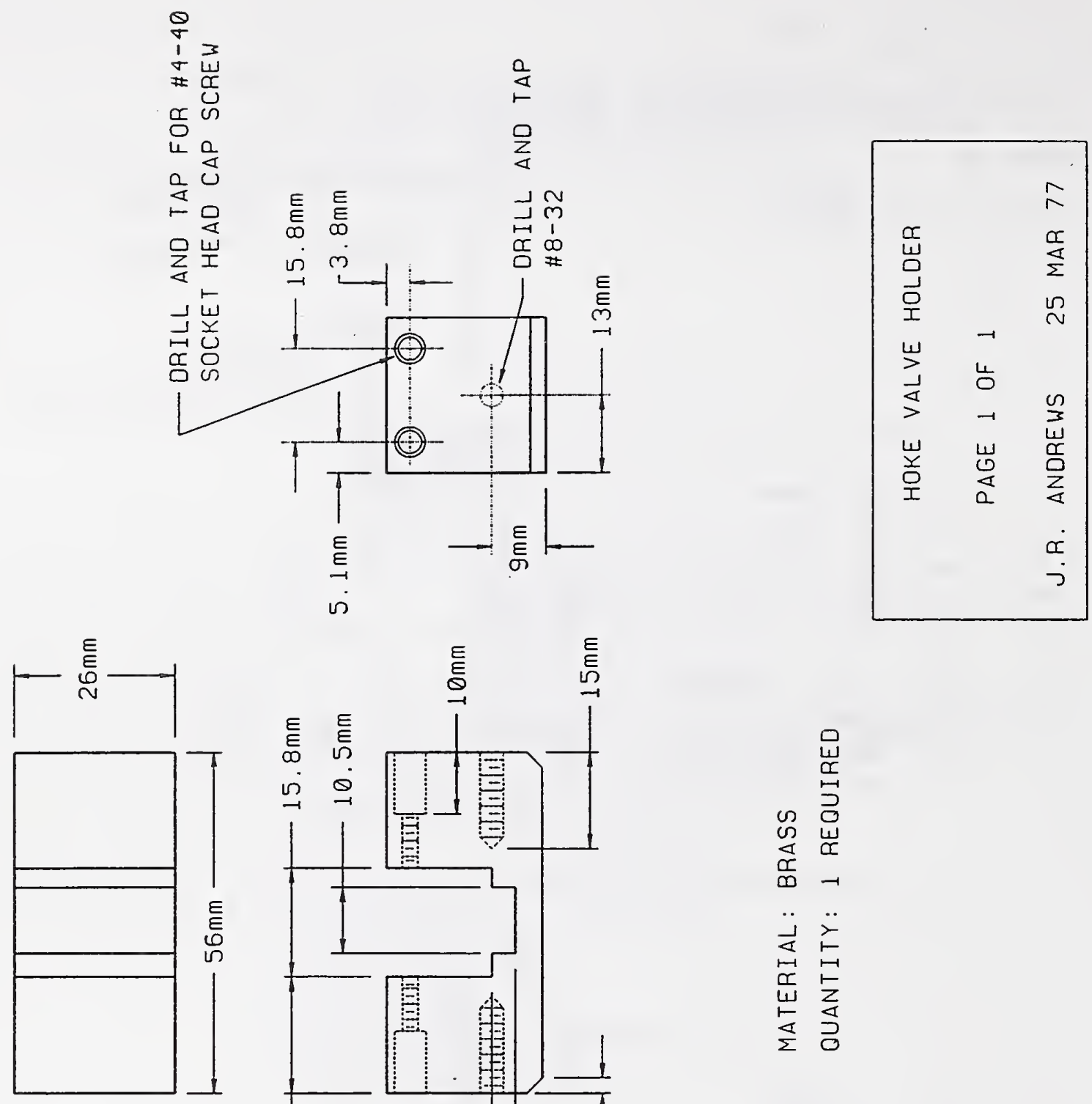
7 Appendix 2:

NBS Pulse Transition Duration Transfer Standard

Temperature Controller 


\section{Debye Line Temperature Controller}

\section{Theory of Operation}

This Appendix describes a temperature controller for the NBS Transition Duration Standards which are based on the Debye-dielectric effect to provide a reasonably well known and reproduceable step-like function when driven from a very fast source such as a tunnel-diode pulse generator. The dielectric liquid used in the standards is sensitive to temperature so it was decided to use electronic means to maintain the lines at $30^{\circ} \mathrm{C}$, which is well above the standard $23^{\circ} \mathrm{C}$ ambient laboratory temperature.

Temperature is monitored on the line-mounting brass block near the end panel with engraved information, that is, model numbers, serial numbers, etc. Two integrated circuits, IC1 and IC5 (each an LM3911), are mounted in physical contact with the brass block. These ICs deliver $1 \mathrm{mV} /{ }^{\circ} \mathrm{C}$ as output. To prevent loading of the outputs, each is buffered by a precision operational amplifier, IC2 and IC6 (each an OP-05A).

The sensor portion of the LM3911 ICs is somewhat different than a thermistor or diode-drop arrangement in that it uses the difference in $V_{B E}$ between two matched transistors with their bases tied together but with widely separated collector currents. As long as the ratio of the collector currents is held constant, a very predictable difference in emitter voltages will occur. This voltage varies with temperature in a known and reproducible manner and is less dependent on processing changes than other semiconductor effects.

This buffered data is then fed via shielded cable to the larger controller box. Some filtering is required at each end of the $1 \mathrm{~m}$ cable to reduce stray noise pickup and radiofrequency interference. The signals are then fed to the inputs of IC3 and IC7 where they are summed with a highly stable reference voltage derived from IC12 which is a temperature-controlled active zener, type $\mathrm{LM} 399 \mathrm{H}$. This voltage is impressed across a series of $0.1 \%$ resistors and switched through the range of $-2.5 \mathrm{~V}$ to $-3.5 \mathrm{~V}$ in $100 \mathrm{mV}$ steps. Small resistors attach to each tap on this range switch to insure a constant impedance source for the buffer IC8. This buffered voltage is applied through a ten-to-one ratio and summed with an equal-magnitude but opposite-polarity voltage from the temperature sensors, producing a nominal output from IC 3 and IC7 of $0 \mathrm{~V}$.

IC7 has a gain set to provide an output of $1 \mathrm{~V} /{ }^{\circ} \mathrm{C}$ as an error voltage. This signal is fed to a comparator circuit consisting of IC10 and IC11 (each LM301) where it is 
compared to a small pair of reference voltages of equal magnitude but opposite polarity. As long as the input remains within the limits defined by these reference voltages, IC 10 will drive Q6 and Q7 through an RC filter and keep the green "GO" bulb lit. When these transistors are conducting, no drive is available for Q8 and the red "NO-GO" lamp remains off. If the error voltage goes out of the set limits, the comparator switches states and since Q6 and Q7 have no drive, the base of Q8 goes up turning it on and lighting the red "NOGO" lamp.

The control channel receives a nearly identical signal as the indicator channel with IC3 summing the temperature signal with the same inverted 10:1 reference voltage. Again, the output should swing above and below $0 \mathrm{~V}$ causing the comparator IC4 to switch on and off and driving Q1 on and off. The collector of Q1 is supplied through a light-emitting diode which provides visual indication of the call for heater drive and also serves as a heater disable function when removed from its socket.

Q1 emitter current is used to energize IC9 (IL-5 or equivalent). The output from IC9 is amplified by the pair of transistors Q2 and Q3 which drive the output switch Q4 and power transistor Q5. This portion of the circuit obtains power from an unregulated supply and is electrically isolated from the critical-circuit areas by the optical coupling provided by IC9.

The heater current controlled by $Q 5$ is sent to the Debye-line package via the $1 \mathrm{~m}$ cable, passing through two $15 \Omega$ resistors in series which serve as heaters, through three $1 \Omega$ resistors in parallel, and then back through the cable to the $30 \mathrm{~V}$ supply. The power resistors are bolted to the Debye line-supporting front and rear brass blocks using heatsink compound to provide good thermal contact. The $1 \Omega$ resistors are mounted parallel to and very near the sensor ICs IC1 and IC5 and the four unused pins on IC1 are bent partially around one of the three $1 \Omega$ resistors. This becomes a sort of thermal feedback arrangement, preheating the control sensor slightly, to overcome the thermal inertia of the large thermal mass to be heated.

The power supplies are conventional circuits, with $+12 \mathrm{~V}$ and $-12 \mathrm{~V}$ furnished by a 7812 and 7912 integrated-circuit regulator fed from a small dual-secondary transformer with each winding rated at $18 \mathrm{~V}$ and $200 \mathrm{~mA}$. The unregulated supply is also a dualsecondary transformer with $1 \mathrm{~A}$ windings connected in parallel to provide more capacity and improve regulation as the heater load is cycled on and off. Use of the separate transformers improves overall stability and minimizes the interaction between the large changes in heater current and millivolt-level signals from the sensor assemblies. 


\section{Calibration Procedures}

Controller Mainframe

1. Verify that entire unit is at laboratory ambient temperature.

2. Disable heater by removing LED from PC board.

3. Remove $1 \mathrm{~m}$ cable to transition duration standard.

4. Turn on and allow 15-20 minute warmup.

5. Turn off. Remove IC8.

6. Using short clip lead, ground TP6, TP4, and TP1.

7. Turn on; null IC7 at TP5; Green light should come on.

8. Remove IC4.

9. Null IC3 at TP2.

10. Turn off; reinstall IC4 and remove IC3.

11. Remove ground from TP1 and ground TP2.

12. Ground base of Q1.

13. Turn on; null IC4 at TP3. (This is a high-gain comparator and the zero crossing may be approximated by watching the meter.)

14. Turn off and remove grounds.

15. Reinstall IC3 and IC8.

16. Remove blue lead "A" from TP8 and place on a ground pin; ground TP7.

17. Turn on; null IC8 at TP6.

18. Turn off and replace blue wire to TP8; remove ground from TP7. 
19. Turn on and check reference voltage at TP6. This voltage should be $-3.0 \mathrm{~V}$ when the front panel control is at $30^{\circ} \mathrm{C}$. The $5 \mathrm{k} \Omega$ potentiometer allows a small amount of correction. A $4 \frac{1}{2}$ digit DVM is necessary.

20. Plug in LED. (It will light if not reversed.)

21. Turn off and reconnect cable to enable calibration of the sensor assembly in the transition duration standard.

\section{Temperature Sensor}

1. Verify that transition duration standard and temperature controller mainframe are connected via the $1 \mathrm{~m}$ cable provided.

2. Remove LED on the temperature controller main chassis.

3. Remove eight screws from the transition duration standard front panel and slide panel forward to access the sensor circuit board inside the standard.

4. Gently slide the circuit board out about $2 \mathrm{~cm}$ and remove the two OP-05 ICs (IC1 and IC5).

5. Turn on the temperature controller.

6. Measure the brass-block temperature at the point shown (see sketch, next page) with a temperature probe to within $\pm 0.1^{\circ} \mathrm{C}$.

7. With a high-impedance DVM measure the voltage to circuit ground at pin 3 of IC1. (See photo, next page, for convenient probe point. Pin 3 is available as the left lead of the front resistor on the circuit card.) Adjust Pot " $A$ ", on right side of circuit board, for correct temperature-to-voltage correspondence @ $10 \mathrm{mV} /{ }^{\circ} \mathrm{C}$, e.g., $22.7^{\circ} \mathrm{C}$ temperature measurement in step 6 means that Pot " $A$ " should be adjusted to yield $227 \mathrm{mV}$ at pin 3 of IC1.

8. Repeat step 7 for pin 3 of IC5 adjusting Pot "B" on right side of circuit board.

9. Turn temperature controller off and reinstall IC1 and IC5. 
10. Remove IC8 on temperature controller main chassis and ground TP6.

11. Turn on; measure voltage at TP1 and adjust Pot " $A$ " on left side of sensor circuit board for the same value measured in step 7 .

12. Measure voltage at TP2 and adjust Pot "B" on left side of sensor circuit board for the same value measured in step 8 .

13. Turn off; replace IC8, remove ground, and replace LED.

14. Turn on; let stabilize to desired temperature, e.g., $30^{\circ} \mathrm{C}$.

15. Monitor voltage at BNC connector labeled "ERROR" at rear of temperature controller with a high-impedance DVM.

16. When system is stable (LED should be cycling on and off) carefully adjust Pot " $B$ " on right side of sensor circuit board for average value of $0.0 \mathrm{~V}$. Monitor this measurement for several minutes and readjust Pot " $B$ " slightly if necessary.

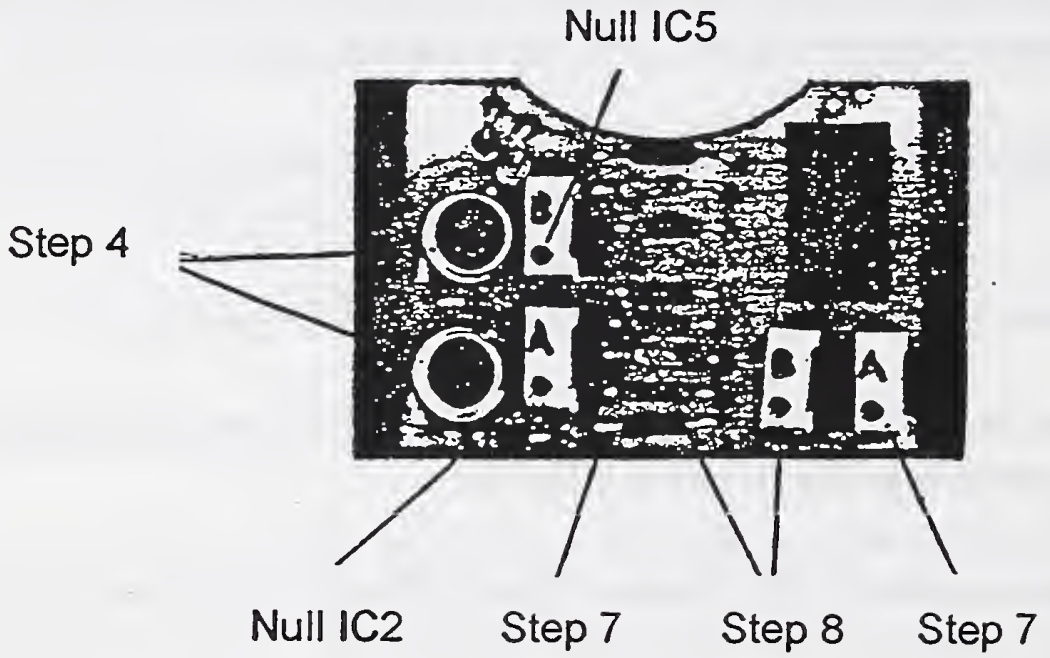

Measurement/adjustment sketches; $A=$ Heater operating point; $B=$ Indicated temperature.
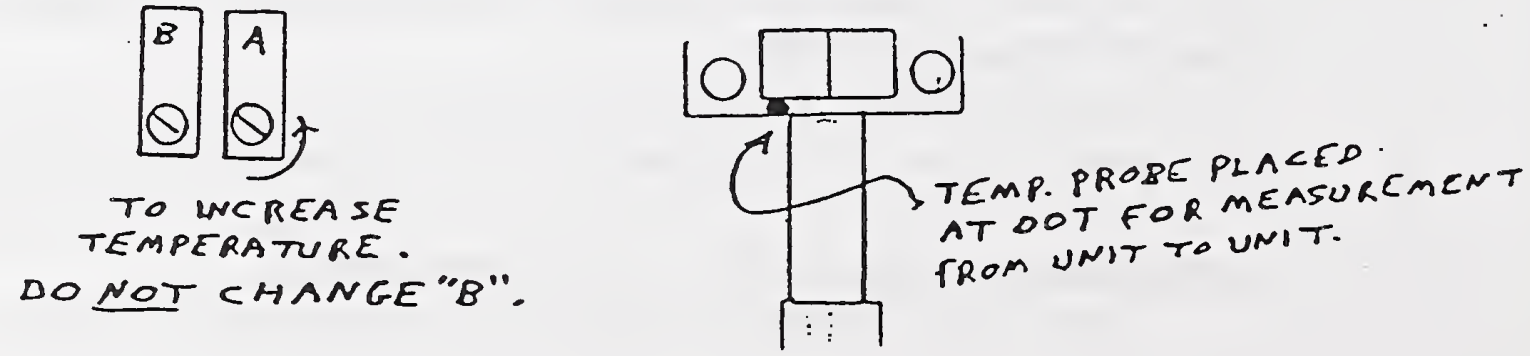


\section{Notes}

In most laboratory situations, it will probably be necessary to readjust the control loop such that the desired operational temperature is maintained. Since the entire Debyeline package, including the case and external devices (samplers, air lines, etc.) acts as a large heat sink. As a result, only a small change in ambient temperature can be compensated for by the controller. Most of the development work for the controllers has been done with the lines near $30^{\circ} \mathrm{C}$ and a laboratory ambient of near $23^{\circ} \mathrm{C}$. However, occasionally the laboratory temperatures varied over a range of approximately $18^{\circ} \mathrm{C}$ to $25^{\circ} \mathrm{C}$. From these variations it was experimentally determined that readjustment of the temperature controllers was often required if the laboratory ambient temperature changed more than about $1.5 \%$.

Typically, the controller is set to $30^{\circ} \mathrm{C}$ and allowed to stabilize. Then, a reference spot is picked as mentioned in the Temperature Sensor calibration procedure and shown on the previous page. Choosing a consistent point for temperature-probing is a requirement because large temperature gradients exist in the overall package. The chosen spot is then monitored and, if necessary, Pot " $A$ " in the transition duration standard is adjusted in small increments until the desired operating temperature is attained.

At this point, the voltage at TP5 should cycle above and below $0.0 \mathrm{~V}$ with a positive value indicating a low temperature and a negative voltage indicating that the temperature is too high.

Due to minor differences in components, the error voltage does not always track the nominal $300 \mathrm{mV}$ signal from the sensor; a simple way to move the center point of the error signal (and therefore the green "GO" lamp) is to adjust the offset pot on IC7 a small amount while monitoring the voltage level at TP5 or the rear chassis BNC connector marked "ERROR."

The schematic diagram of a simple calibration test fixture is included at the end of this appendix. 


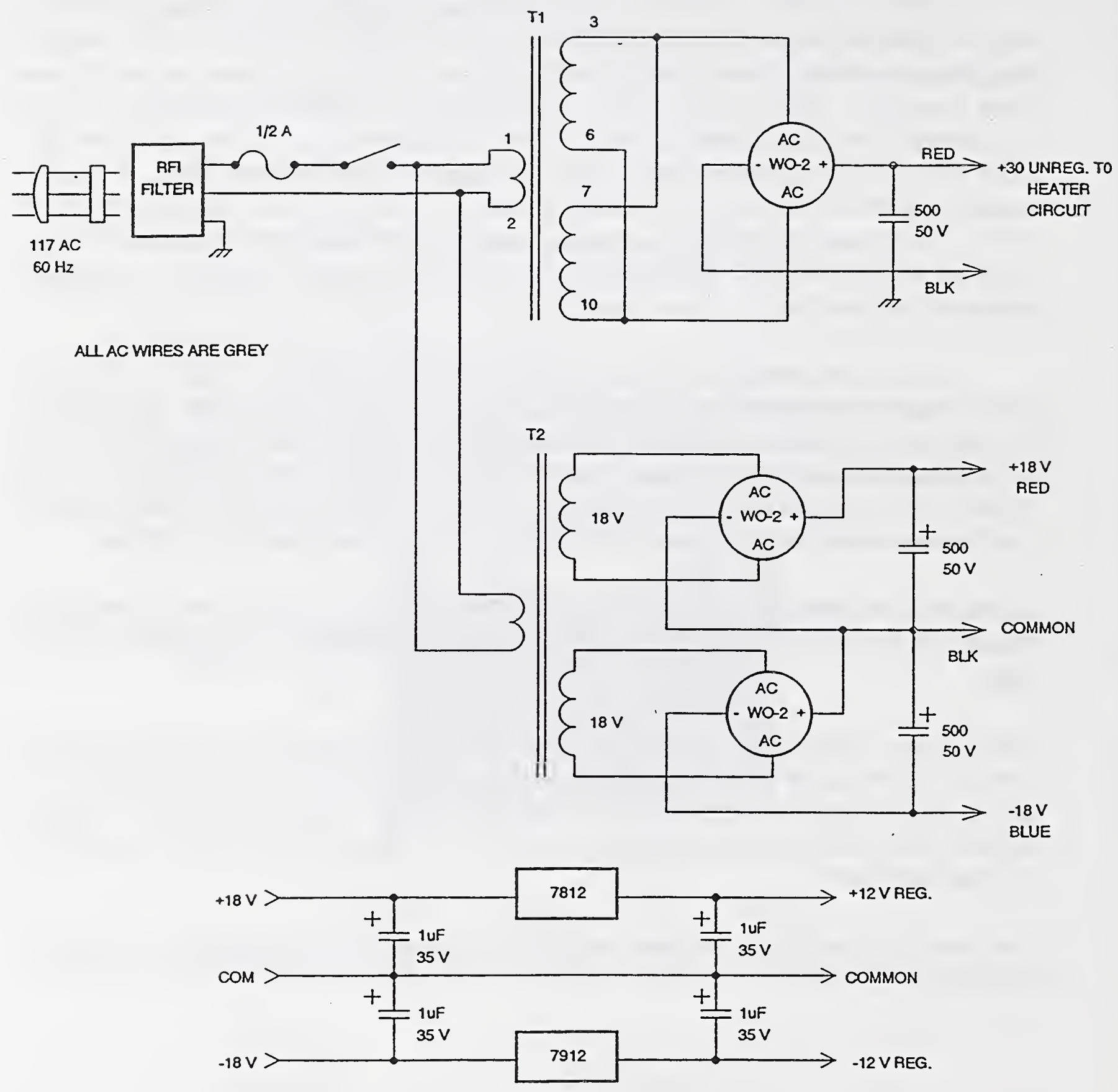

Temperature Controller Power Supply 


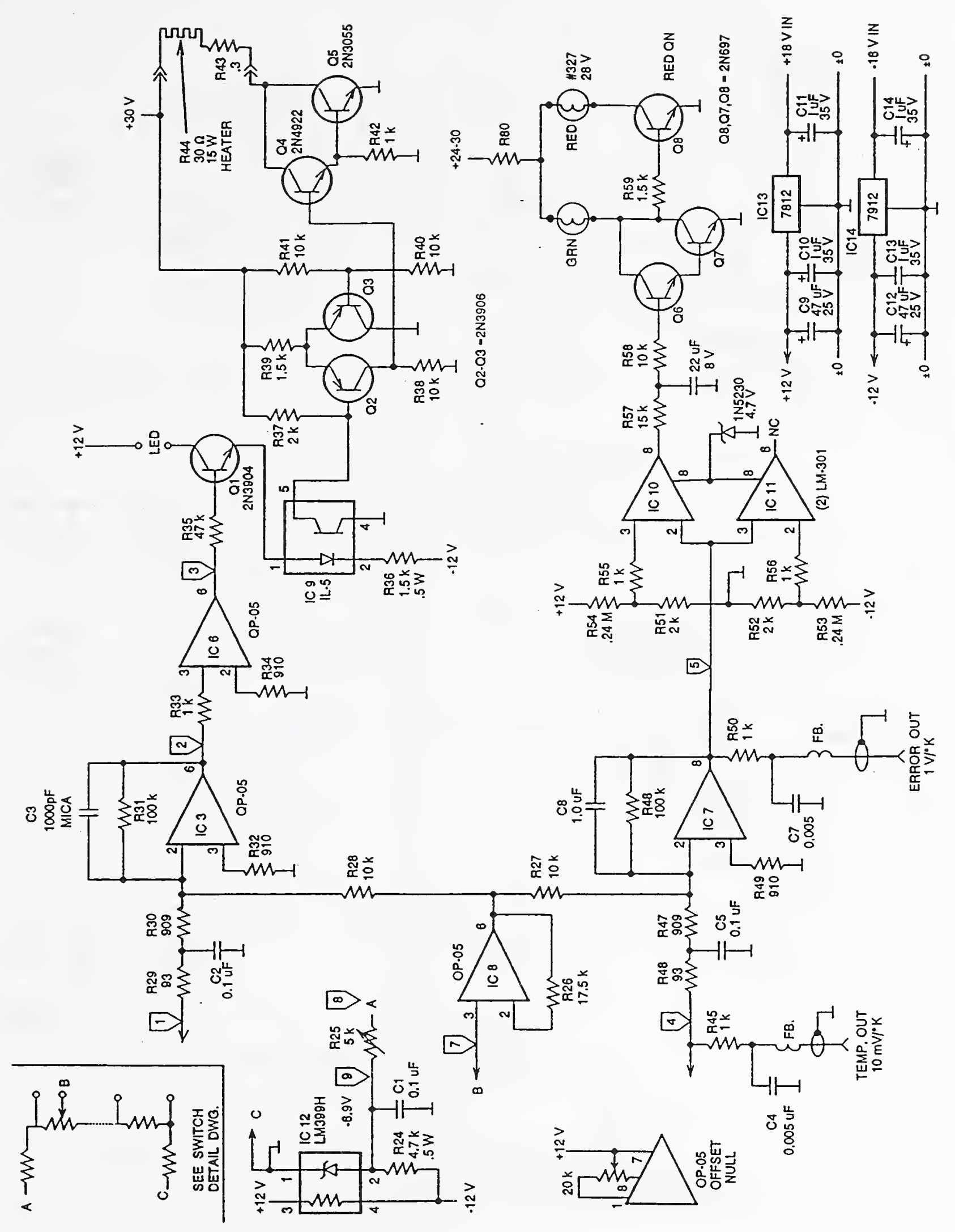

Temperature Controller Temperature Control Board 


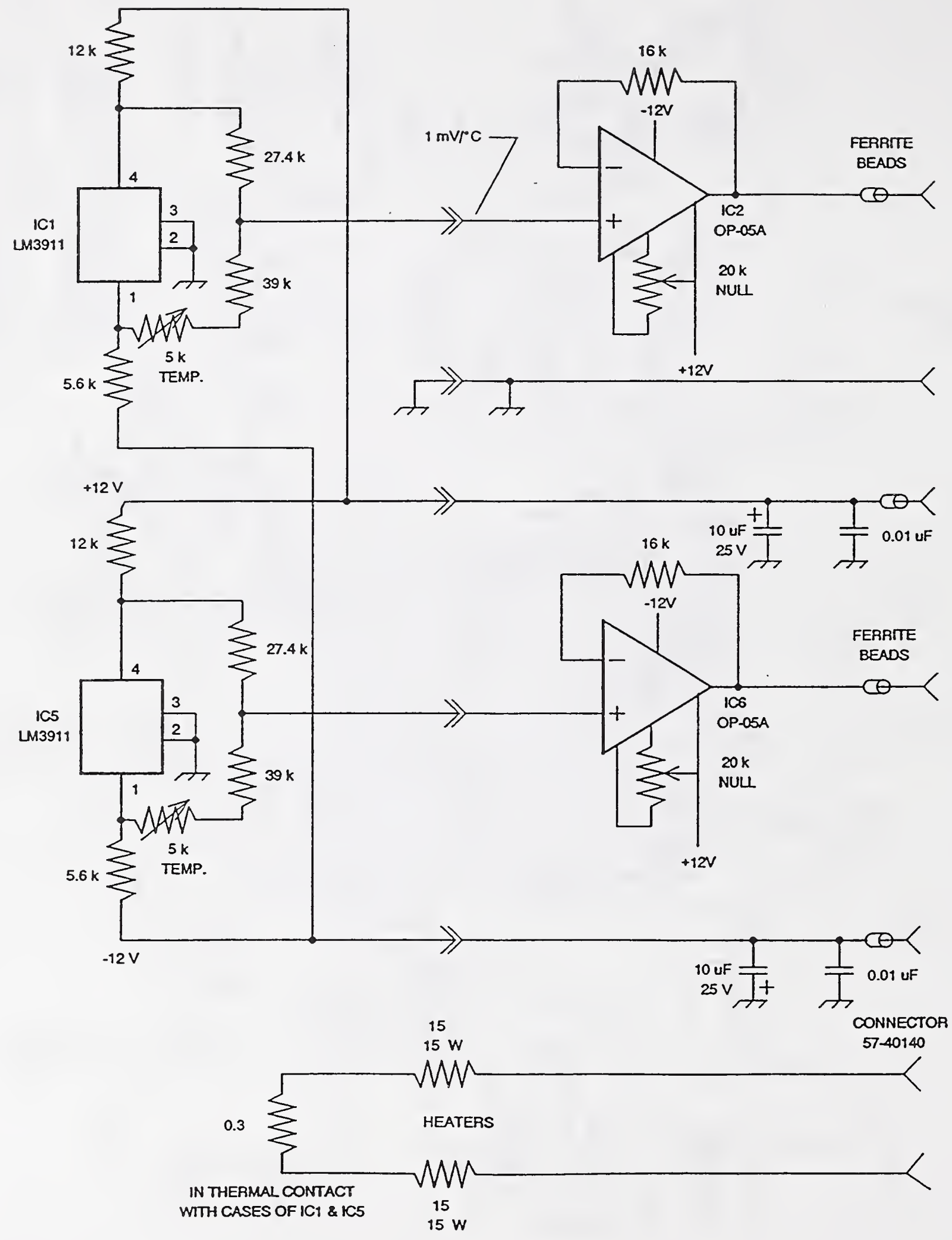

Temperature Controller Control Head Electronics 


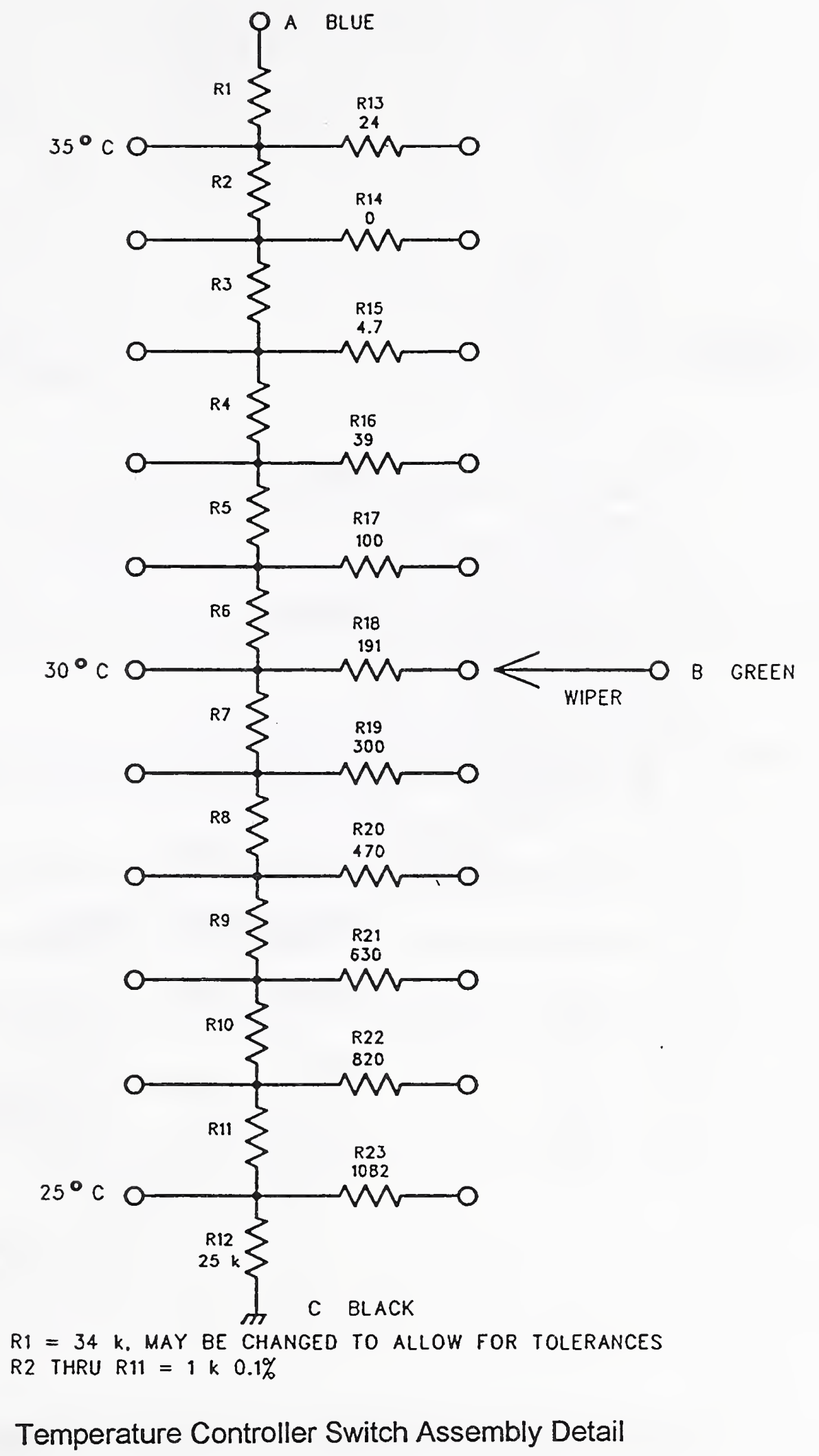


AMPHENOL-CINCH

$57-30140$
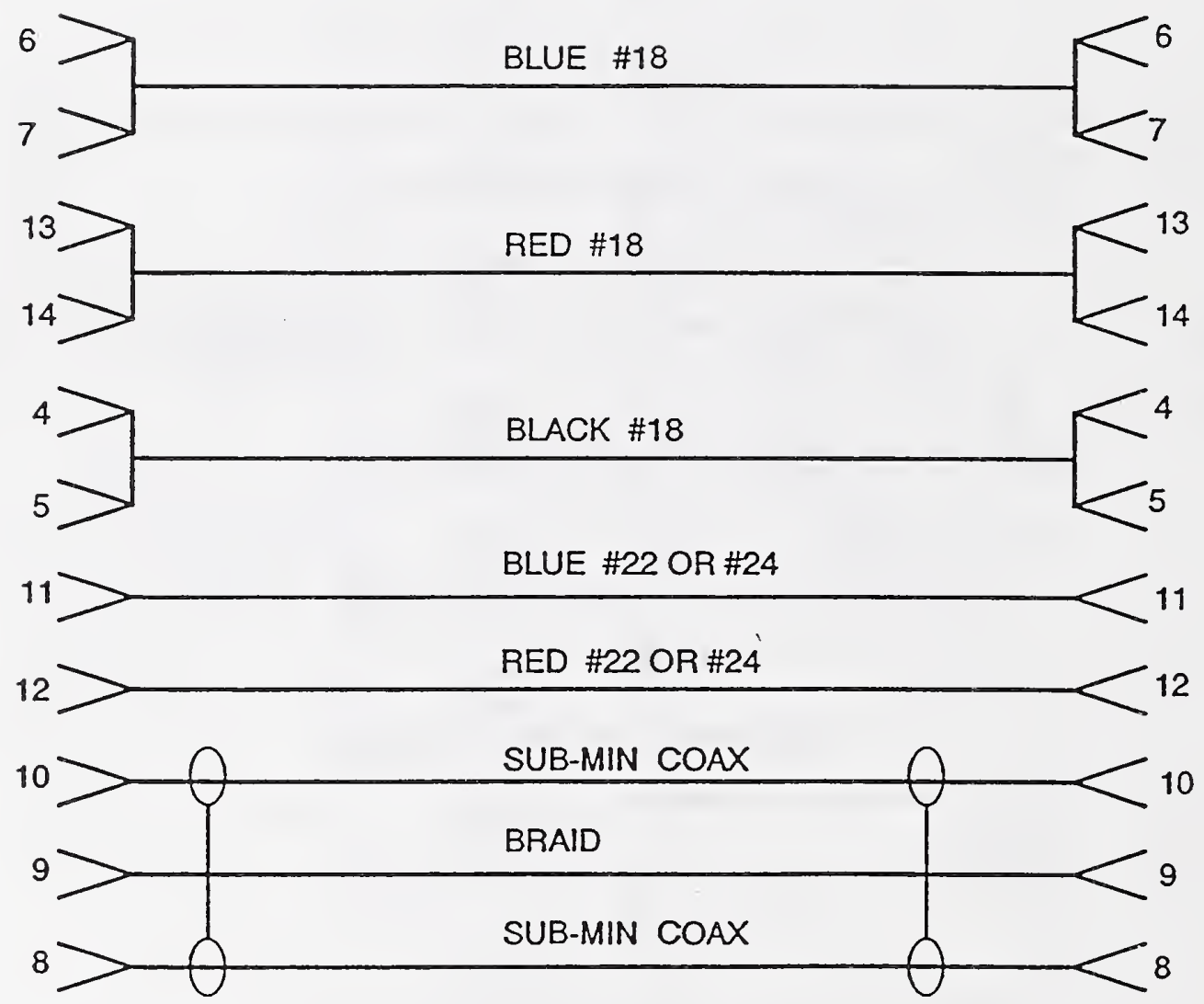

Temperature Controlier $1 \mathrm{~m}$ Interconnect Cable 

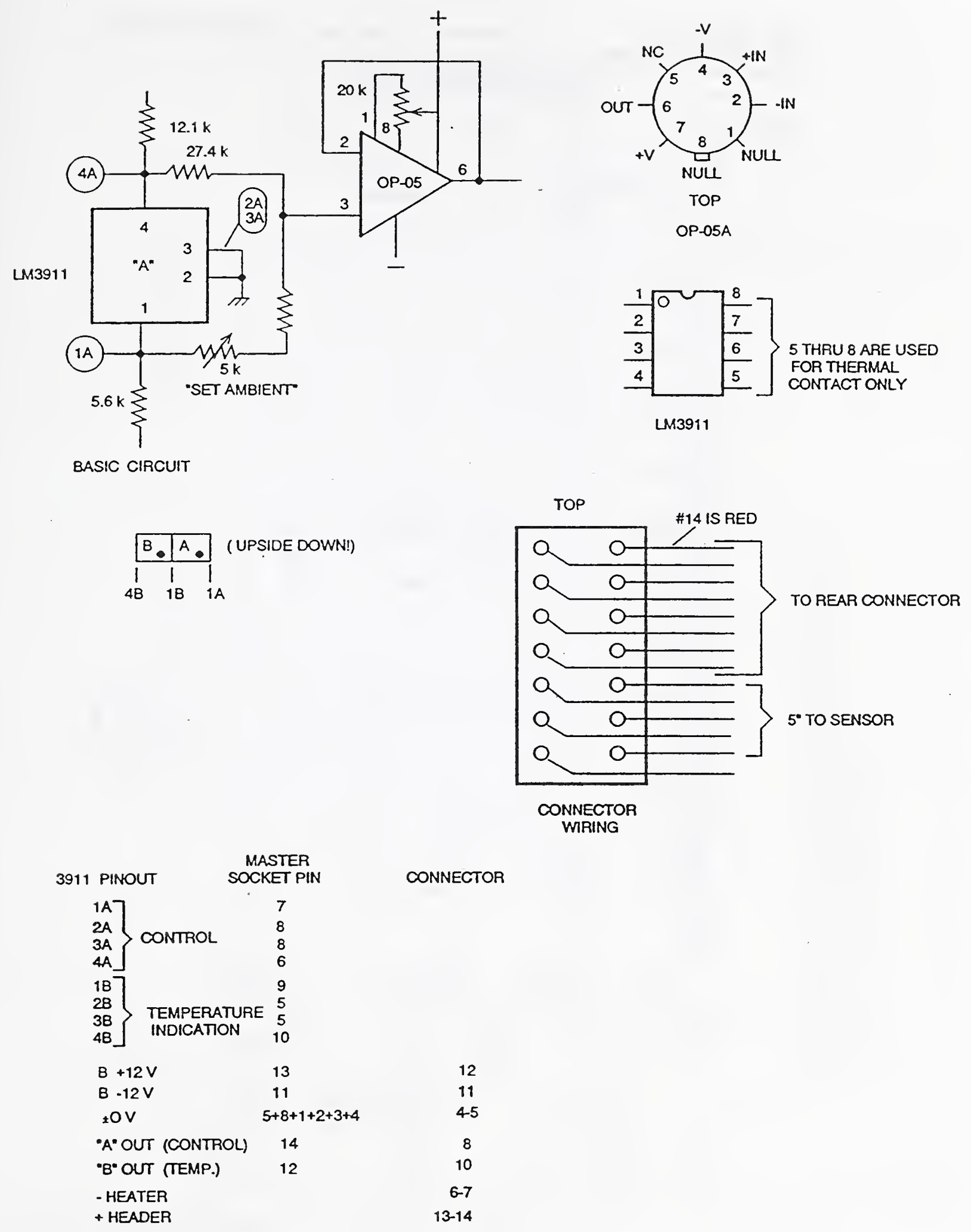

Temperature Controller Connector/Device Pin Configurations 
LET LM-399-H HAVE I $\mathrm{MA}$.

$12 \mathrm{~V}-6.9 \mathrm{~V}-5.1 \mathrm{VFOA} 1 \mathrm{~mA}, \mathrm{R}-5.1 \mathrm{k}$ USE $4.7 \mathrm{k}$ STD. VALUE

LET DMDER CURAENT = 100 UA

LET MID-POINT $Z$ oIf $=17.25 K$ BUFFER TO SEE CONSTANT SOURCE

"A" IMPEDANCE AND PROVDE LOW Z our

$-6.9 \mathrm{~V}$ BLUE TP8

NOMINAL

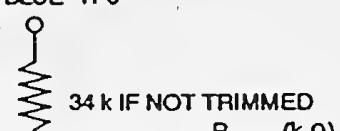

$3.5 \mathrm{~V}$

34KIFNOT TRIMMED

$$
1 \mathrm{k} \sum
$$

$1 k \sum$

InW

$1 \mathrm{k}$

$\sum M M-0$

$1 k \sum$

$\{M M-0$

$1 k \xi$

$\{M W-0$

EFF
17.303

$\triangle R O$ STD. VALVE Q

$1 k \xi$

$\{$ W

$1 k \sum$

$\sum_{1 k} M-0$

$\sum$

$\sum_{k}$

$k$

$1 k$

FMNO

17.327

24

24

0

17.322

4.7

$k \sum$

SMM

$k \leq$

$-2.5 \mathrm{~V}$

fWW $<1 \quad 16.246 \quad 1081 \quad 1000+82$

$\sum_{\text {GREEN }} 25 k$

$\delta^{\circ} C \quad B^{\circ} \quad \Delta V=100 \mathrm{mV} / \mathrm{STEP}$

BLK

GND

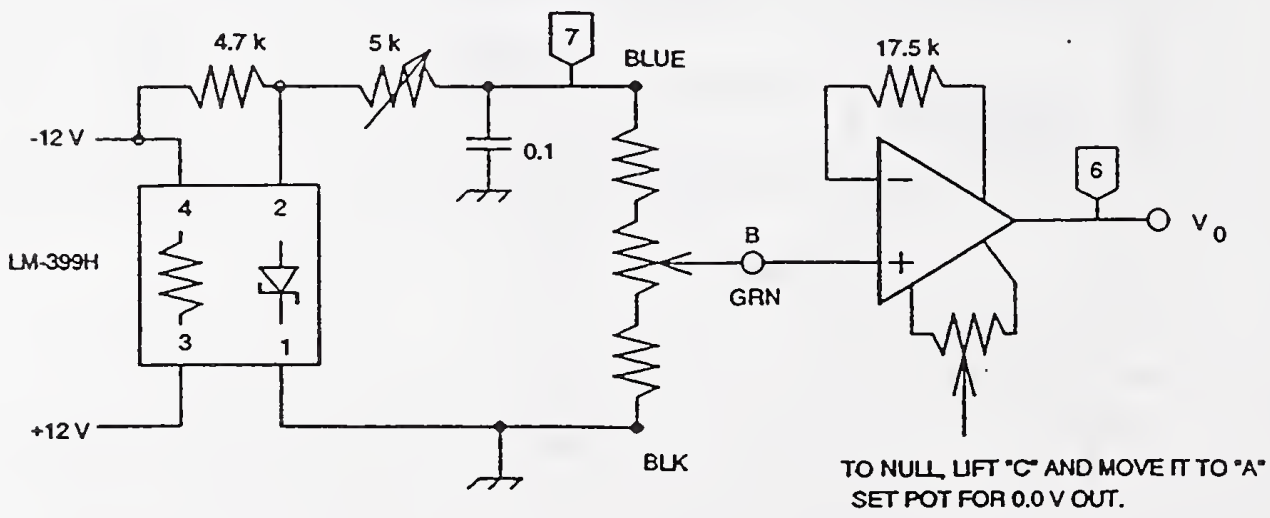

Temperature Controller Voltage Reference 


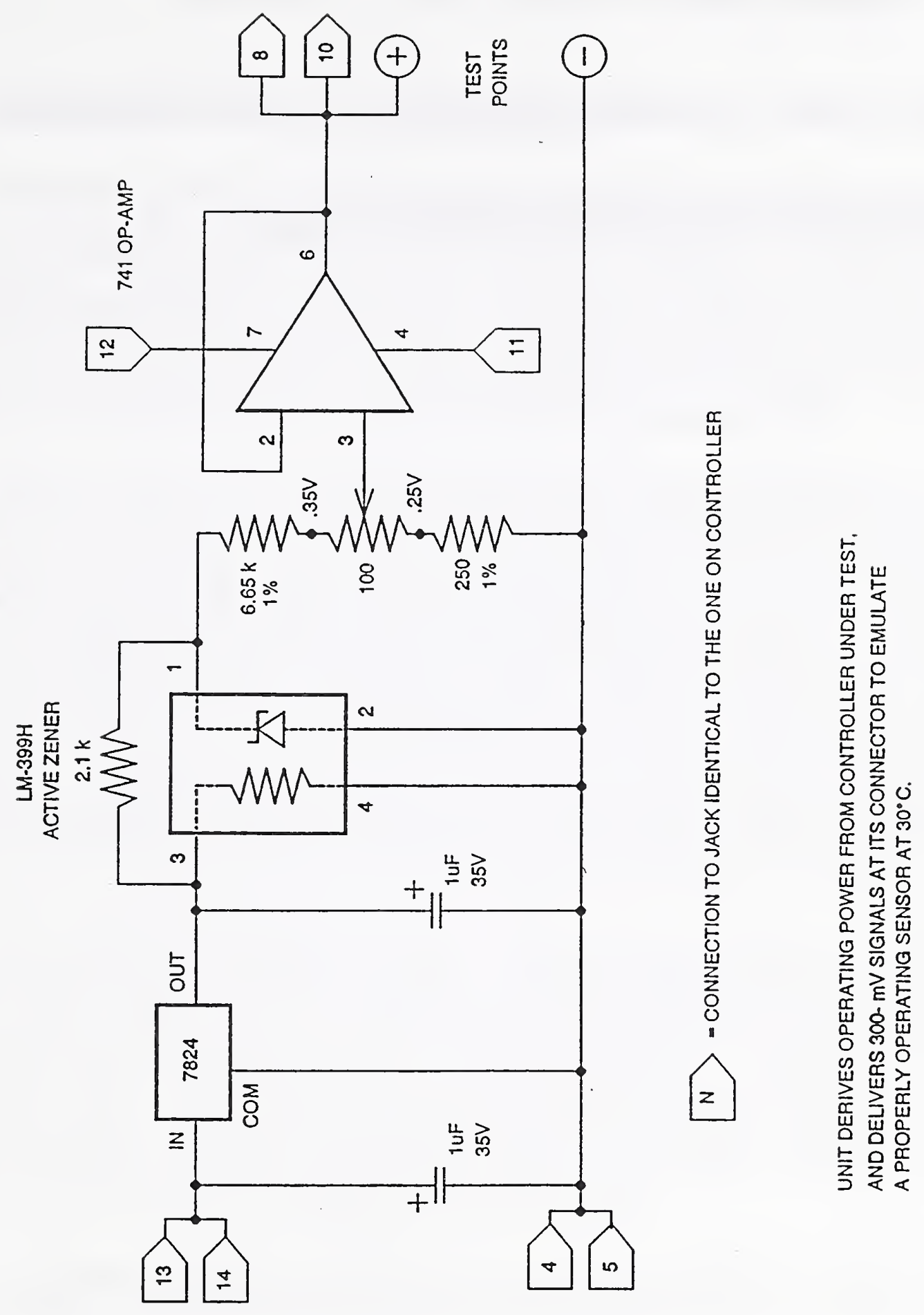

Temperature Controller Calibration Test Package 
8 Appendix 3:

NBS Pulse Transition Duration Transfer Standard

Automatic Tunnel-Diode Bias Supply 


\section{Automatic Tunnel-Diode Bias Supply}

\section{Theory of Operation}

Commercially available tunnel-diode pulse-generator bias supplies suffer from a number of shortcomings; they are usually noisy, leading to excessive time jitter on the tunnel diode (TD) output pulse; they are usually unstable, leading to excessive time drift of the output pulse relative to the trigger pulse; and they add a relatively large drive pulse to the desired TD switching pulse, leading to very poorly defined pulse baselines and toplines. In order to reduce the errors and uncertainties due to these problems, an improved automated TD bias supply has been designed and built at NBS.

The unit is housed in a reasonably large package for ease of packaging and routine maintenance. No effort was made to miniaturize it except in the areas where lead length and layout required this course of action. Where possible, plug-in printed circuit cards were used to insure reproduceability, to allow for improvements as well as to allow having spares for each card. Large, heavy and/or heat producing components were mounted on a common chassis plate which was iridited to improve electrical conductivity. Then, this chassis plate was secured to the metal enclosure which aided in heat sinking. Both top and bottom covers of the enclosure are easily removed to expedite maintenance or modification. Nomenclature on the front panel was engraved by use of a numerically controlled milling machine assuring lasting labels. The computer program has been saved on paper tape. This too provides for reproducing identical panels in the future.

The circuit cards are numbered from 1 through 6 , installed using card guides and designed so that an extender card may be used. All high-speed signals enter and exit the cards via SMA coaxial connectors mounted on the cards themselves, and cables were made long enough to connect to a card even though it might be in the extended position. Card 5 (automatic bias control) and card 6 (trigger pulse generator) use extensive ground plane areas since a fast pulse has spectral components well into the microwave region. Also, the all-metal enclosure helps reduce radiated radio-frequency interference (RFI) while a line filter on the ac input suppresses conducted RFI.

In operation, the pulse repetition rate is crystal controlled by card 4 (logic sequencer) assuring a constant rate. This same card establishes the logic timing for cards 5 and 6. 
The function of card 5 is to measure the point at which the TD was last triggered and bring the bias current up to about $98 \%$ of that value. Then, when a fast pulse from card 6 is superimposed upon this bias current, the TD fires, the point is remeasured, and the bias reset. This occurs at the rate determined by the clock on card 4 . Since a TD heats at the PN junction causing its electrical characteristics to change slightly, additional circuitry was added to provide auto-correction. A switch and variable potentiometer on the front panel provide for automatic or manual correction. Also, there is an external input connector for a user-supplied feedback loop.

The low-voltage power supply (card 1) consists of a multi-tap transformer/diode/filter providing raw dc voltages to integrated-circuit voltage regulators proving $+15 \mathrm{Vdc},-15 \mathrm{Vdc}$, and $+5 \mathrm{Vdc}$, as well as supplying $-18 \mathrm{Vdc}$ via CR7, a zener diode. Because the +5 and -15 Vdc sources must deliver more power, the pass transistor Q1 and the -15 Vdc regulator were mounted off the board for better heat sinking.

The high-voltage supply (card 2 ) is a single-diode bridge and filter fed from a reversed 6.3 Vac filament transformer, T2, whose $6.3 \mathrm{Vac}$ winding is across taps 5 and 6 of T1. With low loading this provides approximately $+200 \mathrm{~V}$ of unregulated dc. A $1 / 8$ A fuse is mounted on this card for protection for the rest of the system to failures in the highvoltage supply.

Card 3 performs two functions by being a high-voltage regulator and also an adjustable current source for a step-recovery diode. IC1 serves as an error amplifier with the inverting input referenced to a zener diode, CR2, and the non-inverting input sensing a sample of the output voltage. The output of IC1 drives Q4 and Q3 setting the bias on pass transistor, Q2. Q5 is included for excess current protection. $A+100$ Vdc source is provided by $Q 6$ and CR8. This supply also acts as a small load on the $+125 \mathrm{Vdc}$ supply, ensuring better regulation.

The current source is provided by sensing a voltage across $\mathrm{R} 12$ and the voltage picked from a $5 \mathrm{k} \Omega$ trimpot across zener reference, CR3. The output of IC2 drives $Q 7$ and Q8 with excess-voltage protection for the tunnel diode provided by diodes CR4 and CR7.

Card 5 contains the automatic bias control circuitry. It operates in the following manner. Q1 and Q2 form a current switch for the TD current supplied by the current source, Q3-Q4. The amount of current supplied is determined by the voltage across the mica capacitor, C10. Op-amp IC1 forces the voltage drop across R2O (and hence the TD current) to be equal to the voltage on C10. The C-RESET line (standing for capacitor reset) sets the voltage across $\mathrm{C} 10$ to $0 \mathrm{Vdc}$ with respect to the $+12 \mathrm{Vdc}$ bus. Q10 is used to discharge $\mathrm{C} 10$ while Q11 discharges $\mathrm{C9}$. 
The MEASURE signal turns on Q6 pulling current from the +12 Vdc reference gradually charging $\mathrm{C} 10$. This action may be observed at TP5. As the charge on $\mathrm{C} 10$ increases, the TD bias follows the increase until the TD switches states, whereupon Q5 goes on and Q6 goes off leaving C10 charged at the point where the TD switched. The TD RESET signal, via Q14, turns Q2 on and Q1 off stopping the bias current and returning the TD to the low state.

The ARM signal turns Q7 on and diverts a small amount (1\% or $2 \%$ ) of the available TD current. The actual amount is determined by Q9 and resistors R33 and R34. ARM also connects $\mathrm{C} 9$ across $\mathrm{R} 20$ and the bias rises rapidly to about the $80 \%$ point, then follows an exponential rise determined by $\mathrm{C} 9$ and R 19 to approximately $98 \%$ of the current required to cause the TD to switch. After a short time a fast trigger pulse is applied to the TD SYNC IN line. This signal is attenuated to a near optimum value by the network R5 through R8 and the $50 \Omega$ trigger line is terminated by R9 and R10. This signal is differentiated by R11 and C3 then applied to the TD BIAS line raising the current enough to fire the TD. The TD BIAS line is terminated by the network R13, R14, L2, R15, R16, and $L 3$ to provide very low reflections.

IC2 and Q9 form a current source which is adjusted by R33 and R34. A reference point is set by IC3 when in the automatic mode and provision is made to offset this value either by a front panel potentiometer or an externally applied voltage via a front panel BNC connector.

C32 provides some protection from noise transients on the TD RESET line. It slows down the action of the differential switch Q1 and Q2 when rearming the TD. This prevents the ARM signal from also triggering the TD.

The impulse generator circuit is found on card 6. The trigger is applied to two 74H0O NAND gates to sharpen up the positive-going transition. The positive-going pulse from the output of the second $74 \mathrm{HOO}$ gate is differentiated by $\mathrm{C} 7$ and is then used to trigger the first avalanche transistor, Q1. When $\mathrm{Q} 1$ avalanches, it very rapidly discharges $\mathrm{C} 8$ through pulse transformer, $\mathrm{T} 1$. The large $(\sim 100 \mathrm{~V})$ pulse from the discharge of $\mathrm{C} 8$ is coupled through the transformer to the second avalanche transistor, Q2. The secondary of the transformer is floating with respect to circuit ground. Thus, terminals 4 and 6 produce, respectively, a positive- and a negative-going pulse, which is coupled to the base-emitter junction of Q2 through the $1 \mathrm{k} \Omega$ resistors $\mathrm{R} 8$ and $\mathrm{R} 9$. This differential drive pulse produces a large turn-on base current for $\mathrm{Q} 2$, and yet it is well balanced with respect to ground such that it produces a negligible signal on the output line. R8 and R9 were chosen to be large compared with $50 \Omega$ to produce negligible loading on the Q2 output pulse. 
When Q2 avalanches, it very rapidly discharges the charge line DL2 into the $50 \Omega$ output circuit. DL2 was previously charged up to the $\mathrm{B}+$ voltage (typically $+125 \mathrm{Vdc}$ ) through the charging resistor $\mathrm{R} 20$. Q2 produces a $50 \mathrm{~V}$ pulse into $50 \Omega$ with a $0.4 \mathrm{~ns}$, $10 \%$-to- $90 \%$ first transition duration. C11 is used to provide some peaking on the leading edge to compensate for the delay line distortion. The pulse duration of about $10 \mathrm{~ns}$ is determined by twice the electrical length of DL2. The 90\%-to-10\% second transition duration of $15 \mathrm{~ns}$ is intentionally made slower by the RC network R7-C10 at the far end of DL2. The $50 \mathrm{~V}$ pulse from Q2 is too large for the SRD used, which will typically have a 25 $\checkmark$ reverse breakdown voltage. Thus, a simple $\pi$-network $6 \mathrm{~dB}$ attenuator is used to reduce the Q2 pulse to an amplitude just slightly less than the SRD's breakdown voltage. RFC3 and RFC4 are used as a bias-insertion network to supply forward-bias current to the SRDs. R17, R18, and R19 are used as a $20: 1$ voltage divider to provide a sample of the driving pulse for use as a trigger output pulse. R18 and R19 in parallel set the output impedance of the trigger pulse at $50 \Omega$.

The $74 \mathrm{H} 00$ gates, $I \mathrm{C} 1$, and the $96 \mathrm{~S} 02$ monostables, $\mathrm{IC} 2$, are used as a maximumrepetition-rate limiter. The circuit limits the maximum repetition rate to prevent damage to the avalanche transistors, Q1 and Q2, due to excessive power dissipation. The positivegoing transition of the input clock pulse, pin 53, is differentiated by R1 and C1. This positive spike is inverted twice and sharpened by gates IC $1 \mathrm{~d}$ and ICIC and is used to trigger Q1. The output of the first gate $\mathrm{IC} 1 \mathrm{a}$ is also used to trigger the first monostable IC2a. IC2a produces a $4.1 \mu$ s pulse which is added together with its triggering pulse in gate IC $1 \mathrm{~b}$ to produce a $5.1 \mu$ s pulse to ensure triggering of Q2. The trailing edge of the $\mathrm{IC} 2 \mathrm{a} 4.1 \mu \mathrm{s}$ pulse is used to trigger the second monostable IC2b. IC2b produces a $3.8 \mu \mathrm{s}$ pulse. The $4.1 \mu$ s and $3.8 \mu$ s pulses are added together in gate IC $1 \mathrm{~b}$ to produce a $7.9 \mu \mathrm{s}$ pulse. R2 and $\mathrm{C} 2$ are a delay network to compensate for the propagation delay through IC2b. IC1a inverts the $7.9 \mu$ s pulse and applies it to the second input of the IC1d gate. This disables IC1d so that it will not accept any additional input clock pulse for $7.9 \mu \mathrm{s}$, thus preventing any further triggering of Q1 and Q2 until the end of the $7.9 \mu$ s period.

In addition to that of the circuit cards, the remaining circuitry is also shown in the attached figures. A majority of the front-panel wiring is shown including the cables used to interconnect the high-speed areas of the circuit. Switch S2 energizes a small coaxial relay used to choose either external or internal synchronization. A second section of S2 switches two LEDs as a reminder to the user of the switch position.

Choice of internal, external, or automatic drift correction is make by S3. One section of S3 connects the dc input port of card 5 to either a BNC panel jack for external control, a small variable voltage source controlled by a 20 -turn potentiometer, or leaves it floating allowing the automatic control loop to function. Since the only switches in stock 
were of a double-pole, double-throw type with center 'off', a small logic circuit was included to activate LEDs for use as indicators. This assembly mounts under the main chassis plate.

The second section of S3 is used to ground the LED cathodes when in the EXT or INT position. A 7400 NAND gate has its inputs tied to the anodes of the LEDs so that the open or AUTO position occurs only during the time both gate inputs are alike. Q2 provides inversion and buffering to the green LED. A small diode in the 7400 ground lead moves its effective ground to about $+0.6 \mathrm{Vdc}$ in order to assure $T T L$ levels at the inputs since the normal LED drop is above the usually required $+0.8 \mathrm{Vdc}$.

\section{General Notes}

1. An error in layout of card 4 caused the pin number indication to be reversed, i.e., " 1 " and "60" are transposed.

2. R35 located on the top edge of card 5 is used to set the nominal TD current. This may be done in the INTERNAL mode and with the multi-turn front panel potentiometer set to mid-range.

3. Be sure card 5 fits in the socket properly. Some cards were sheared a bit small and misalignment can occur resulting in open-circuit conditions.

4. Test pins were not installed for the $+12 \mathrm{Vdc}$ or the $+15 \mathrm{Vdc}$ points.

5. There is no TP3.

6. The original artwork for card 5 omitted a trace between the junction of R44 and R45 to pin 21 of the edge connector. A small jumper was added rather than rework the boards. 


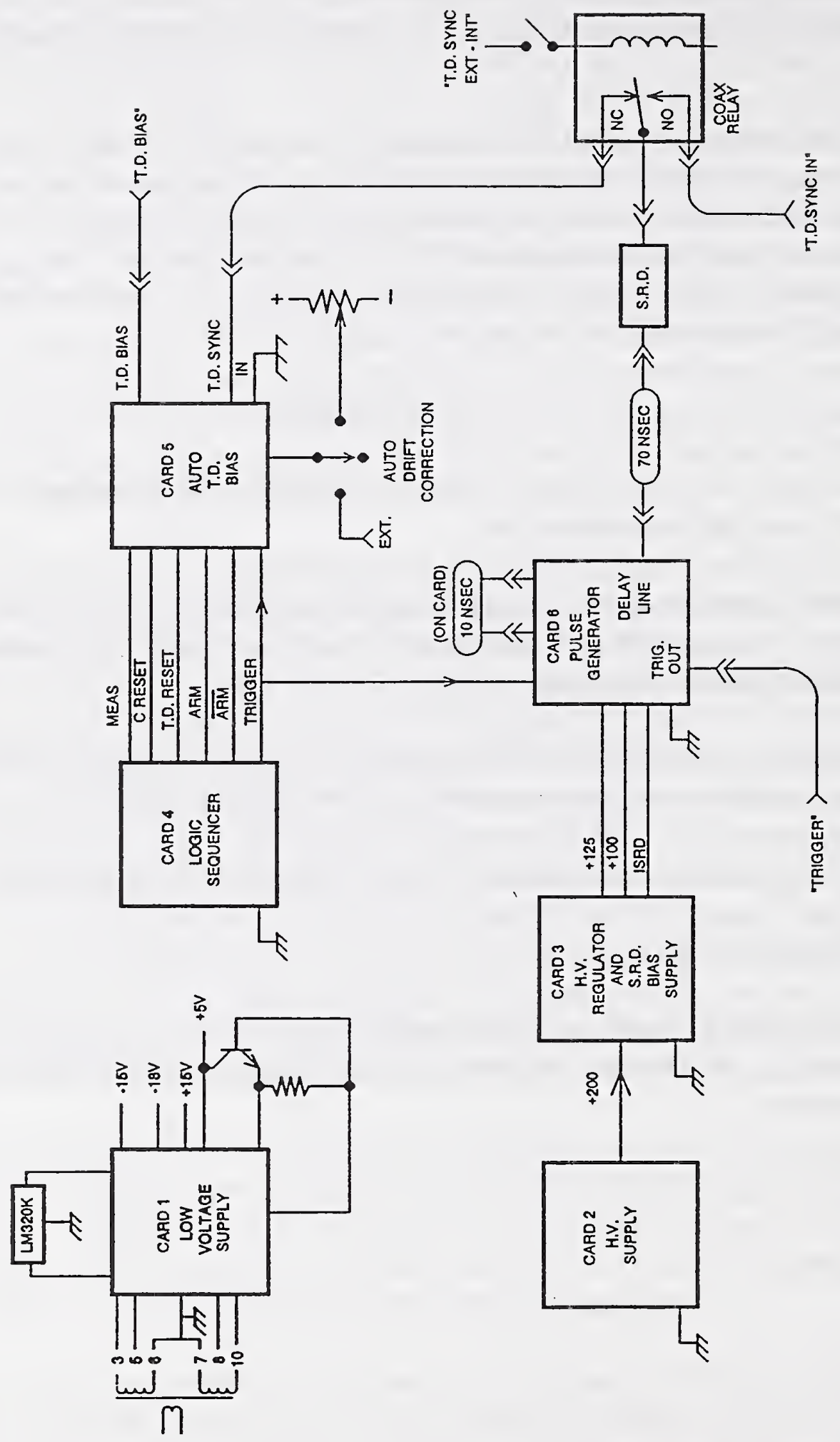

Automatic TD Bias Supply Block Diagram 


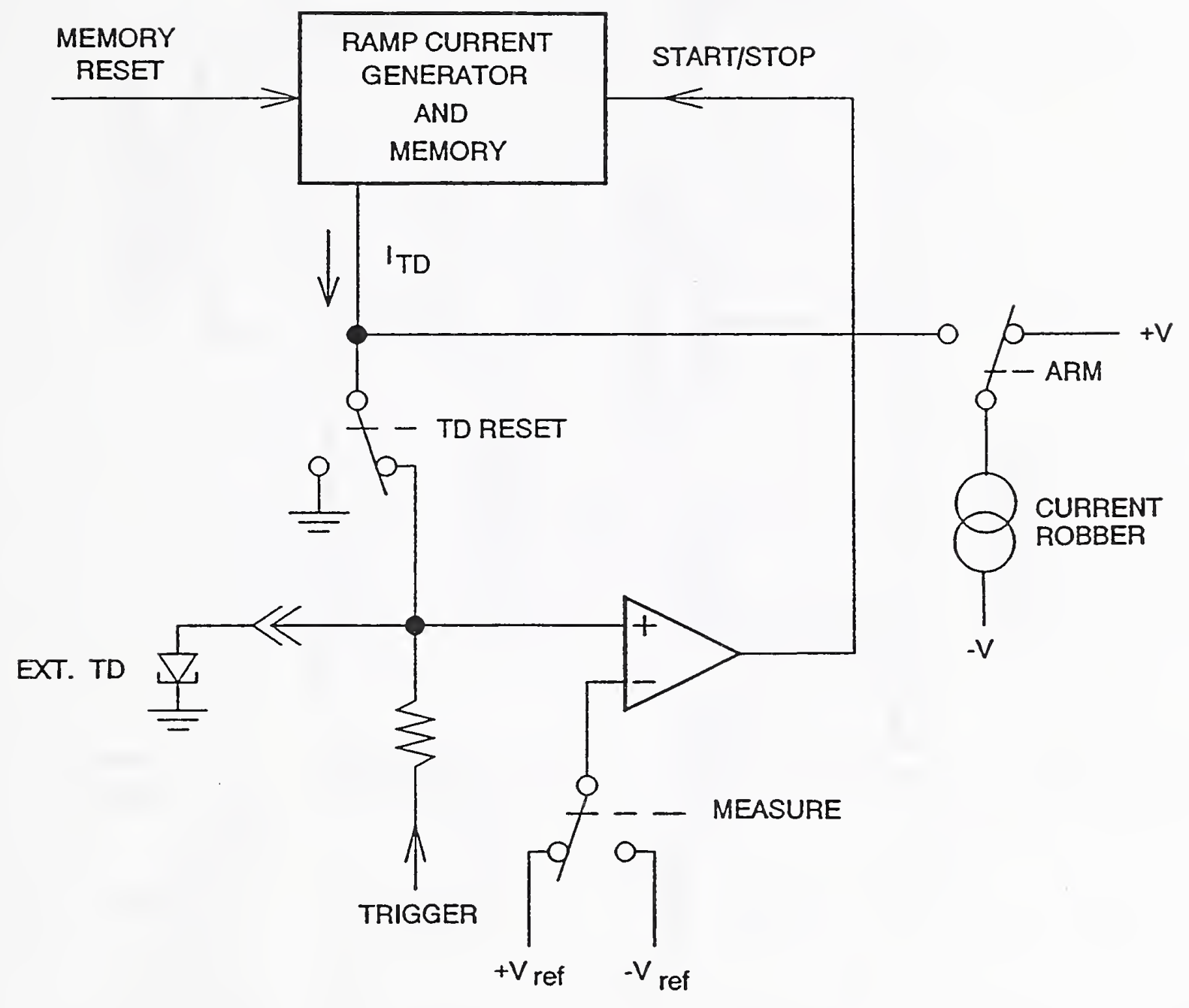

Automatic TD Bias Supply Operation Diagram 

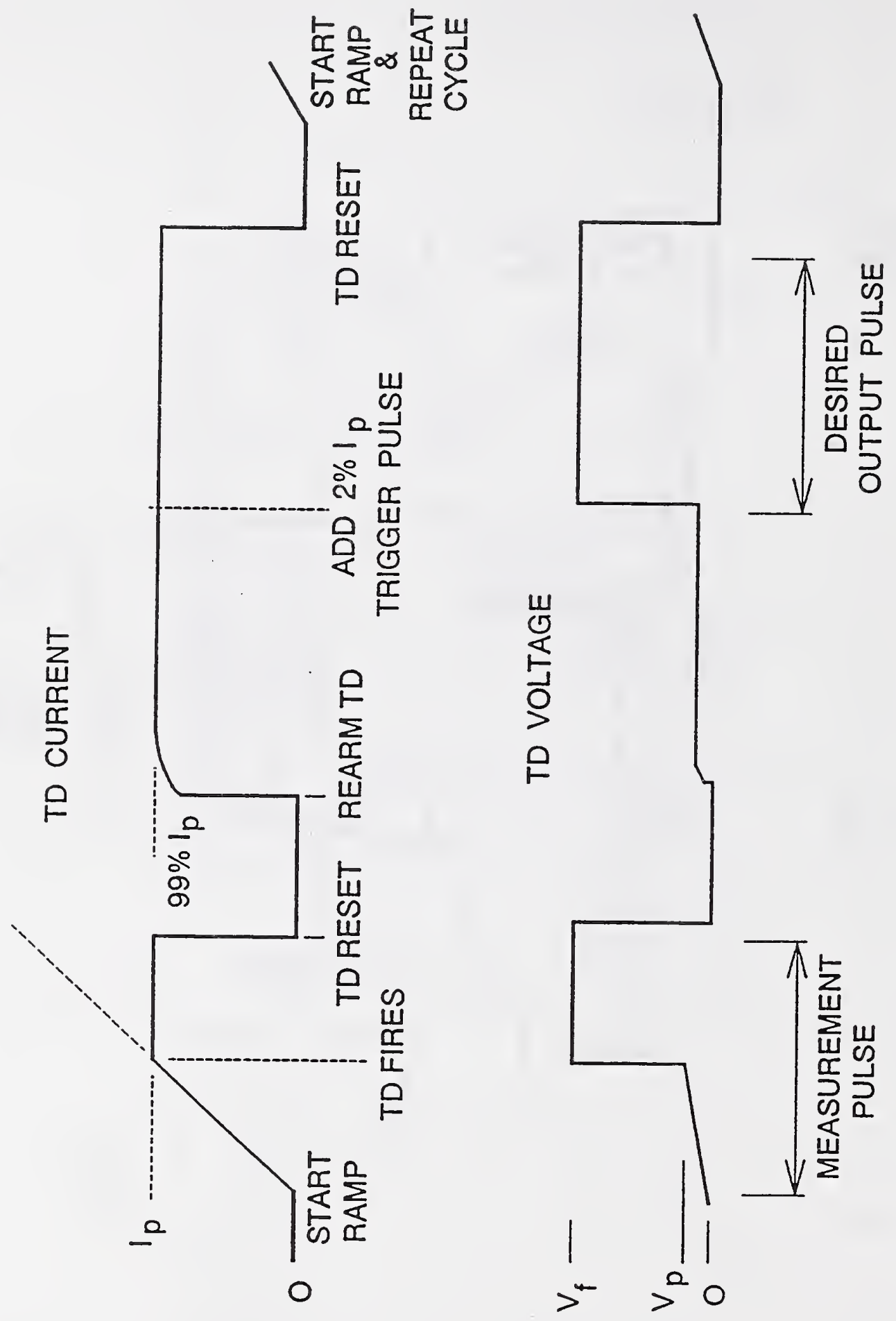

Automatic TD Bias Supply Basic Principle of Operation 


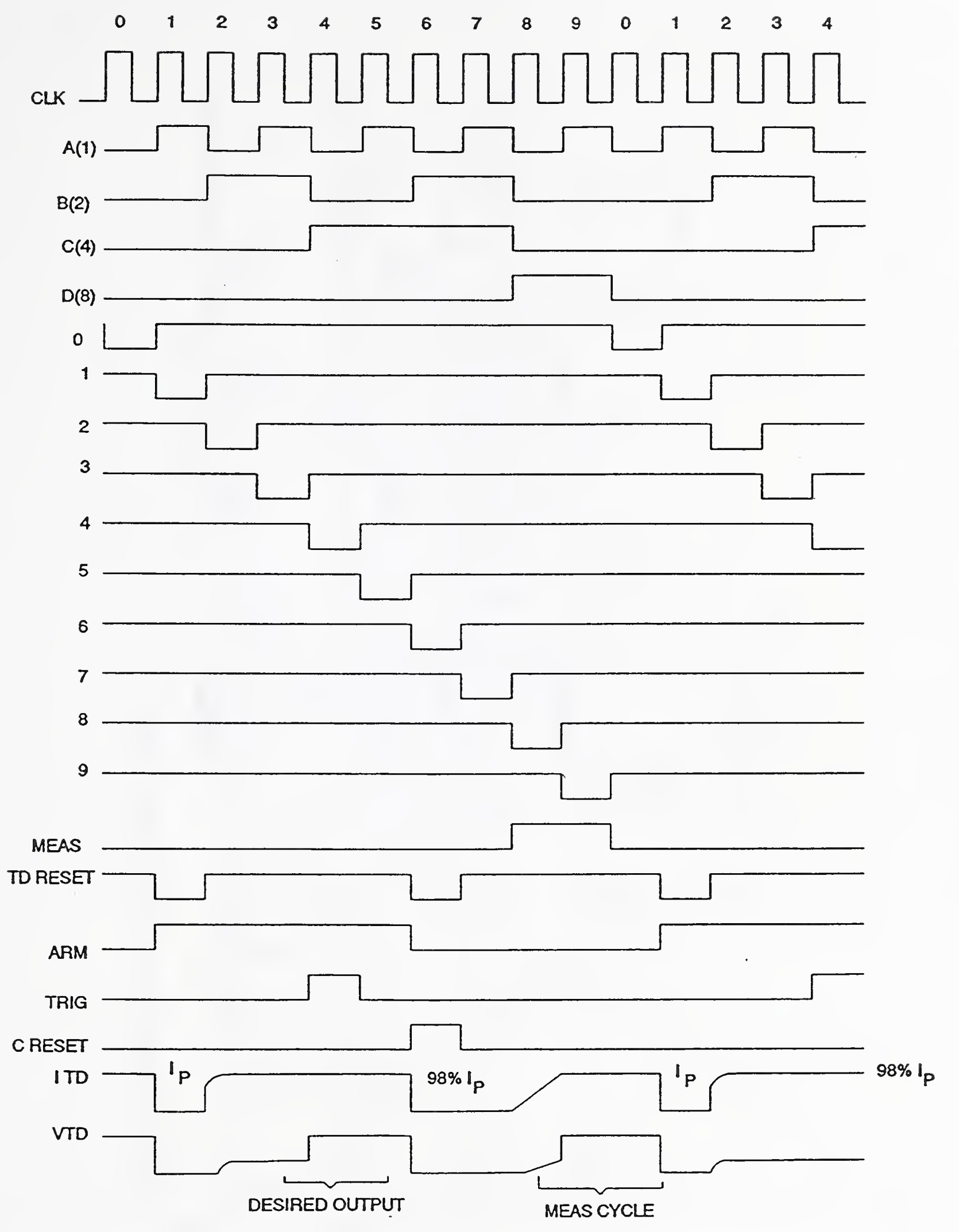

Automatic TD Bias Supply Logic Sequencer Timing Diagram 


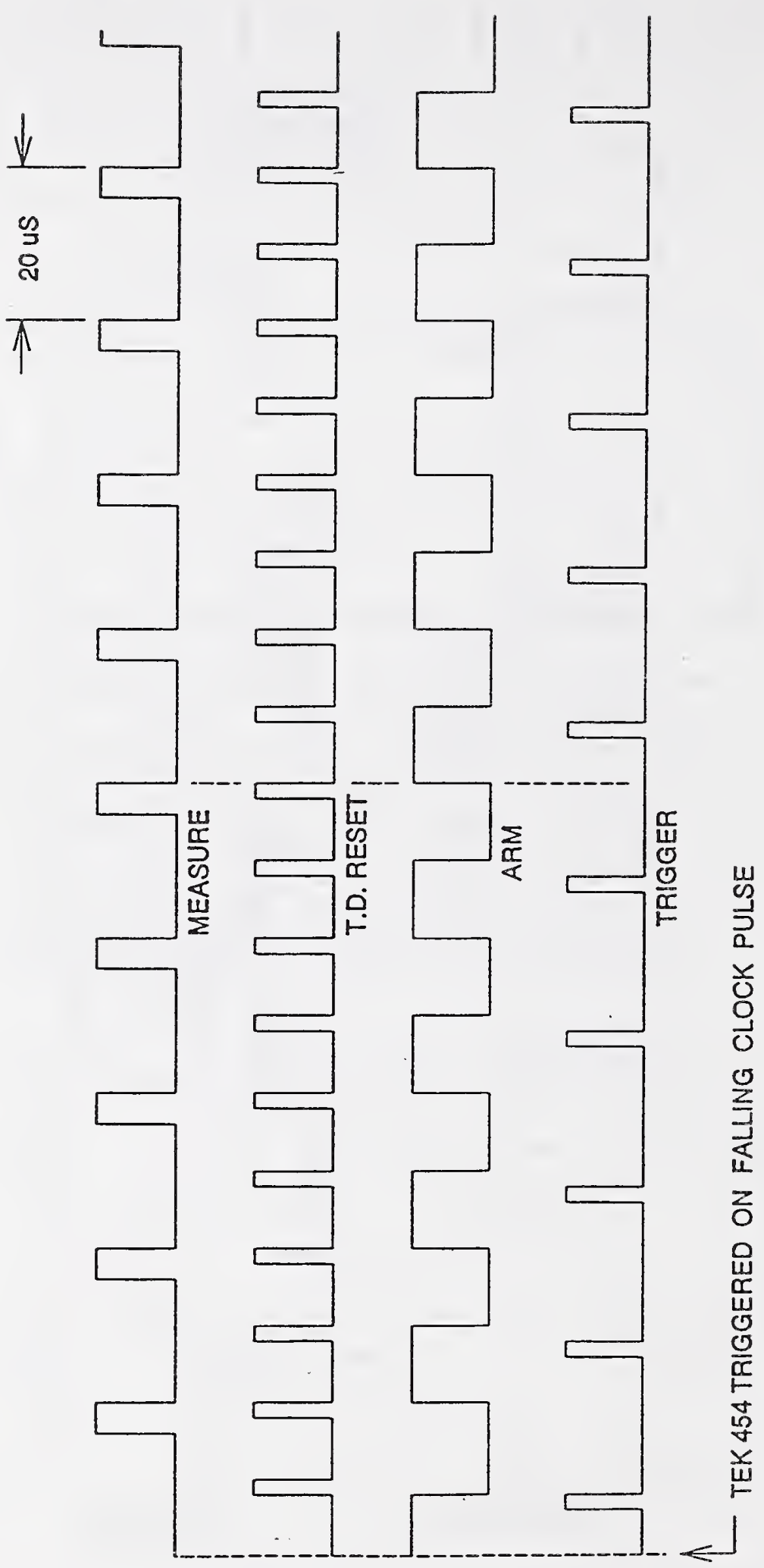

Automatic TD Bias Supply Logic Sequencer Board as seen on Logic Analyzer 


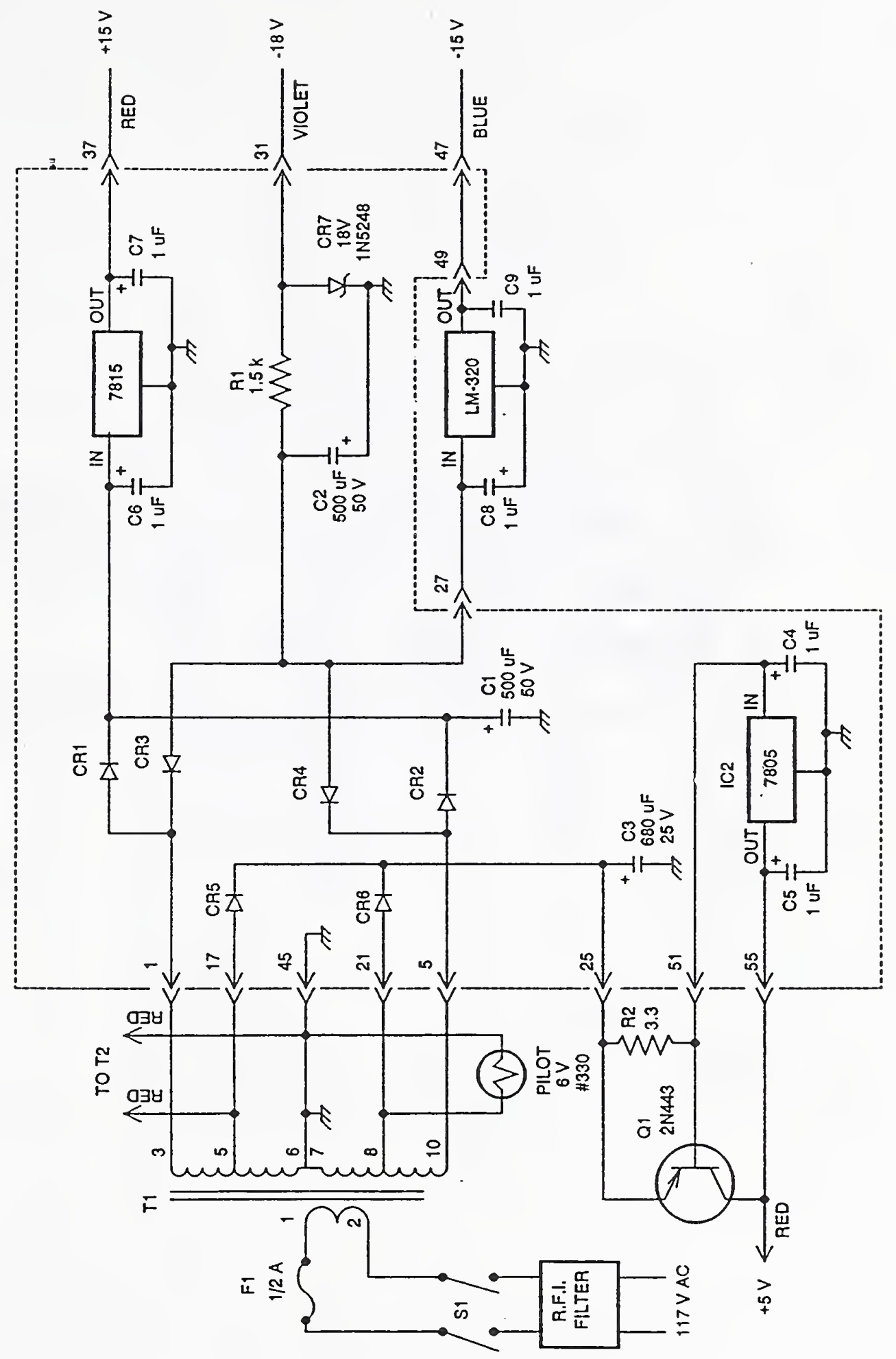

Automatic TD Bias Supply Low-Voltage Power Supply 


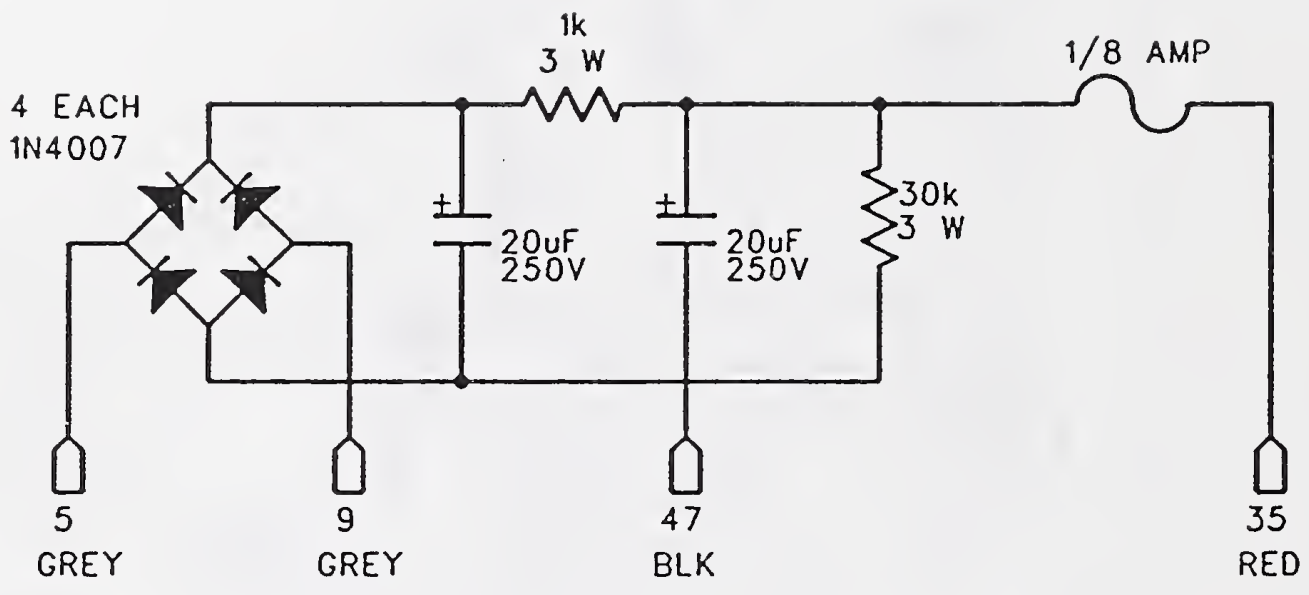

Automatic TD Bias Supply High-Voltage Card 


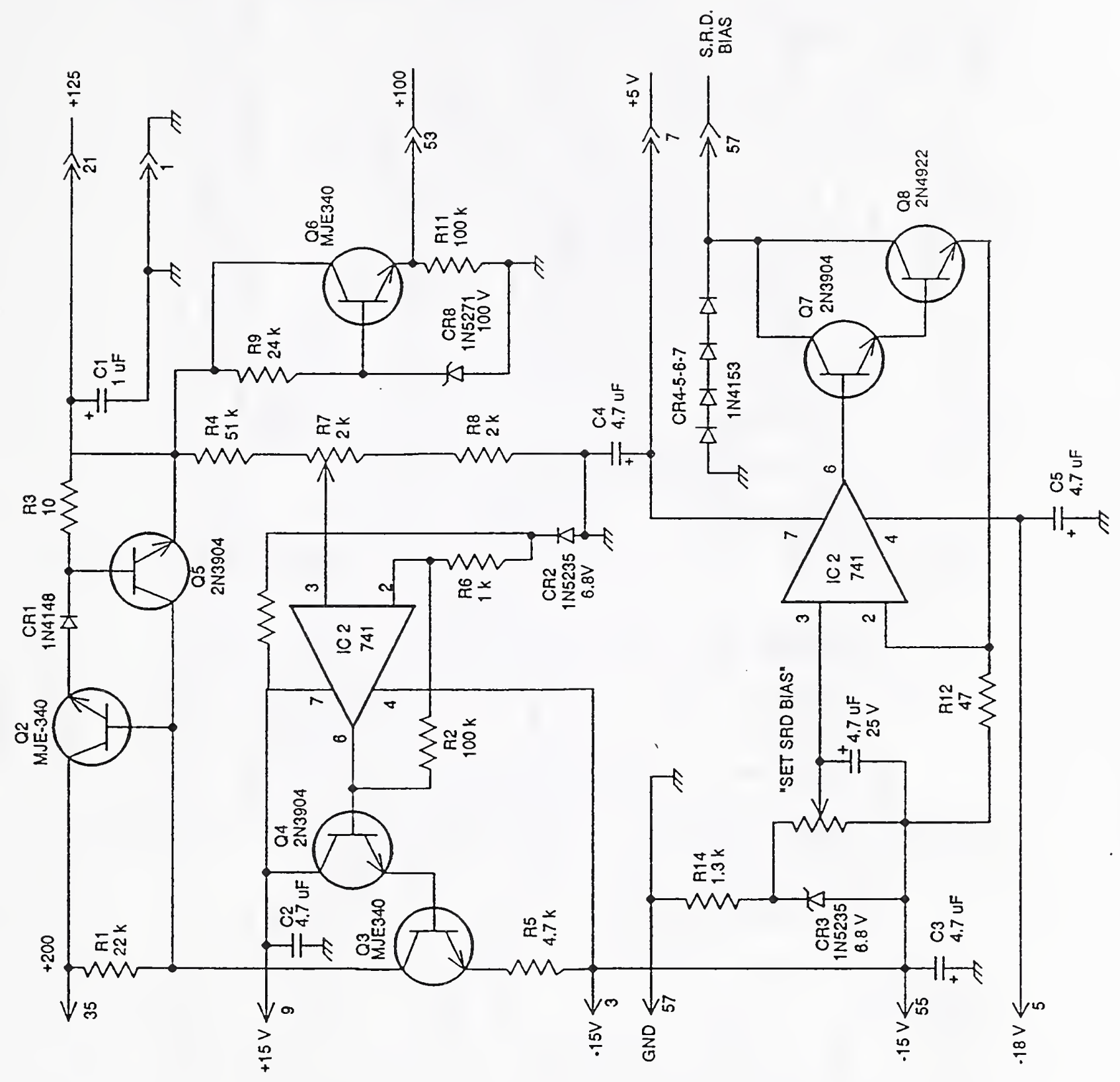

Automatic TD Bias Supply HV Regulator and SRD Bias 


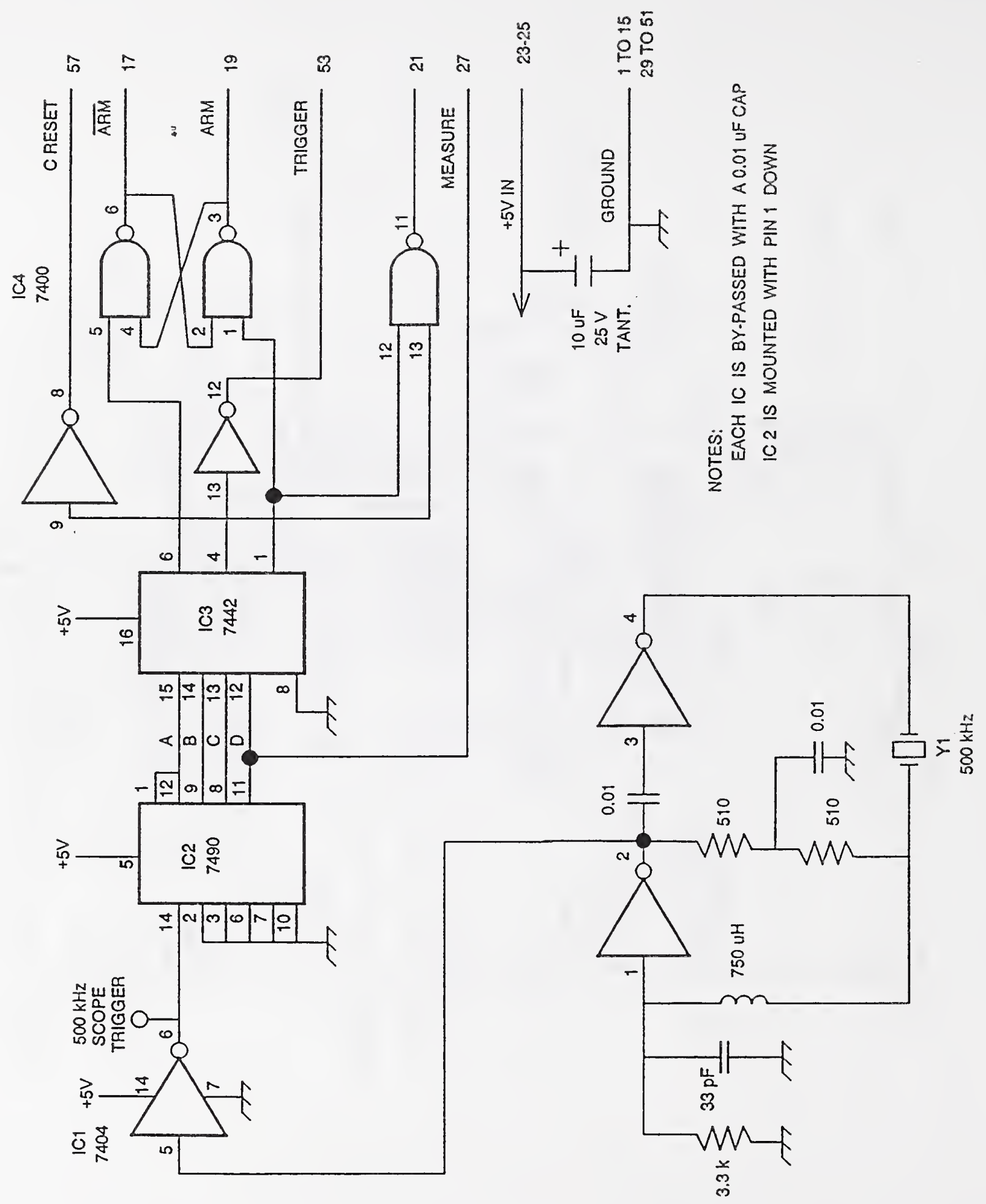

Automatic TD Bias Supply Logic Sequencer 


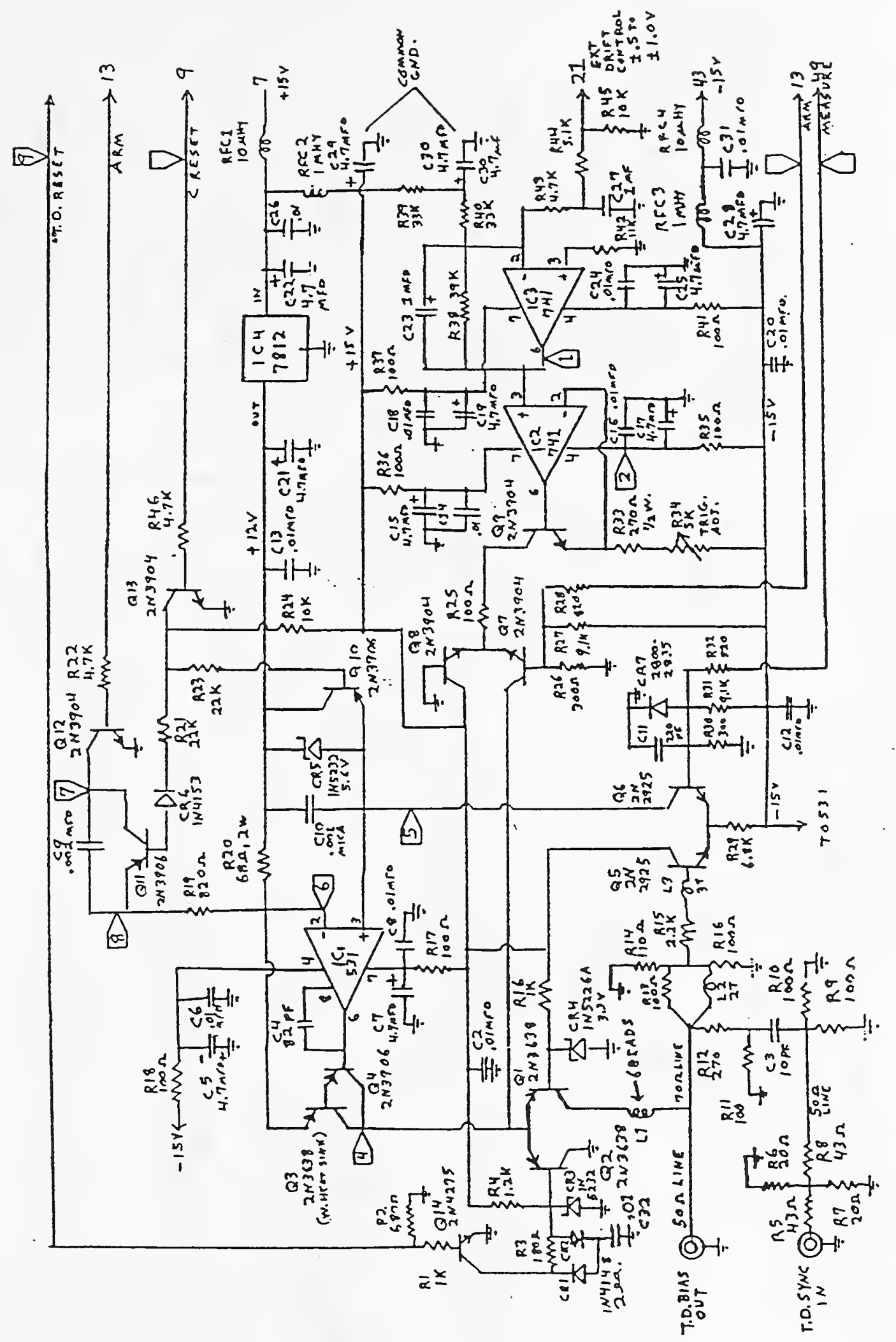

Automatic TD Bias Supply Automatic TD Bias Control 


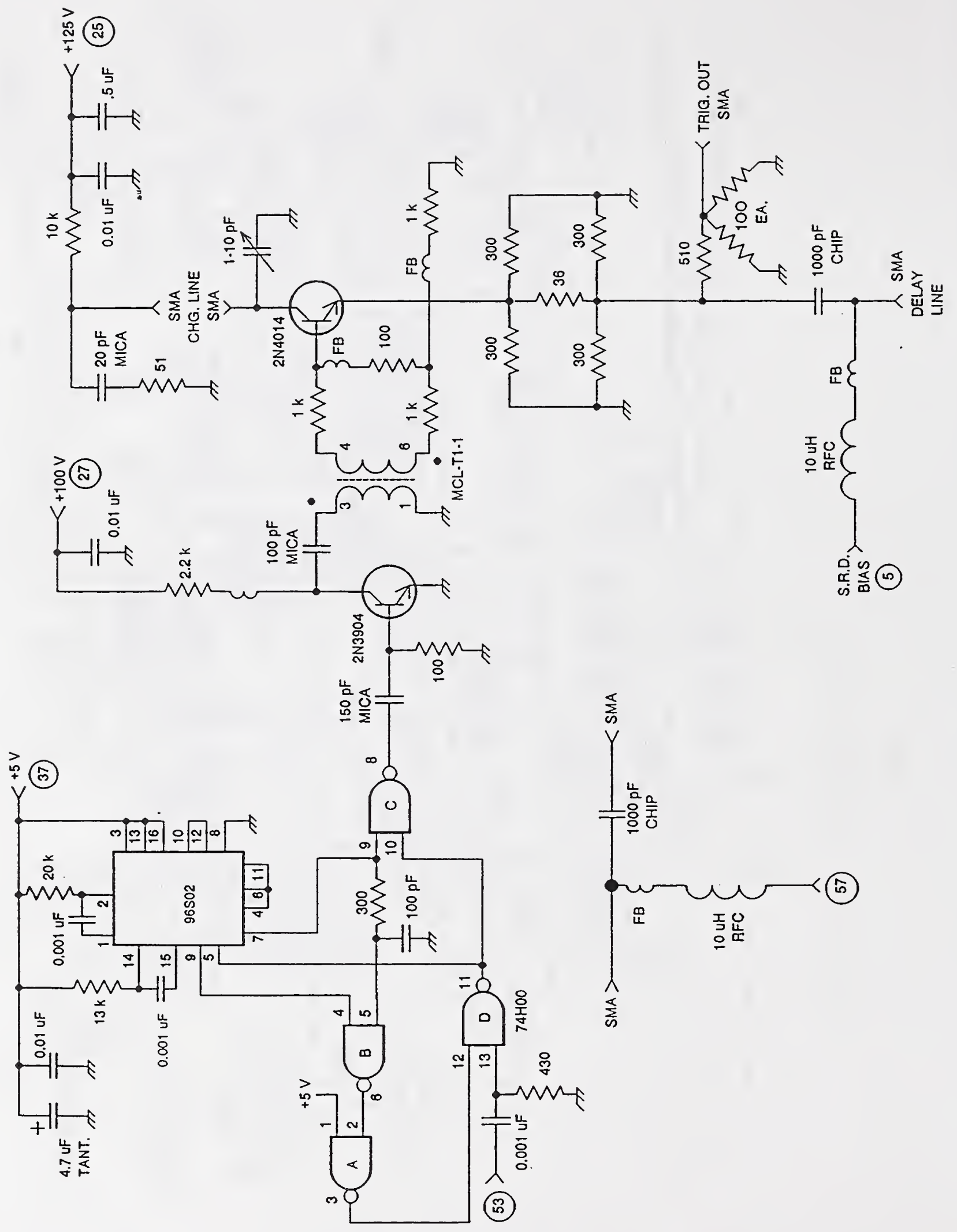

Automatic TD Bias Supply Impulse Generator Card 


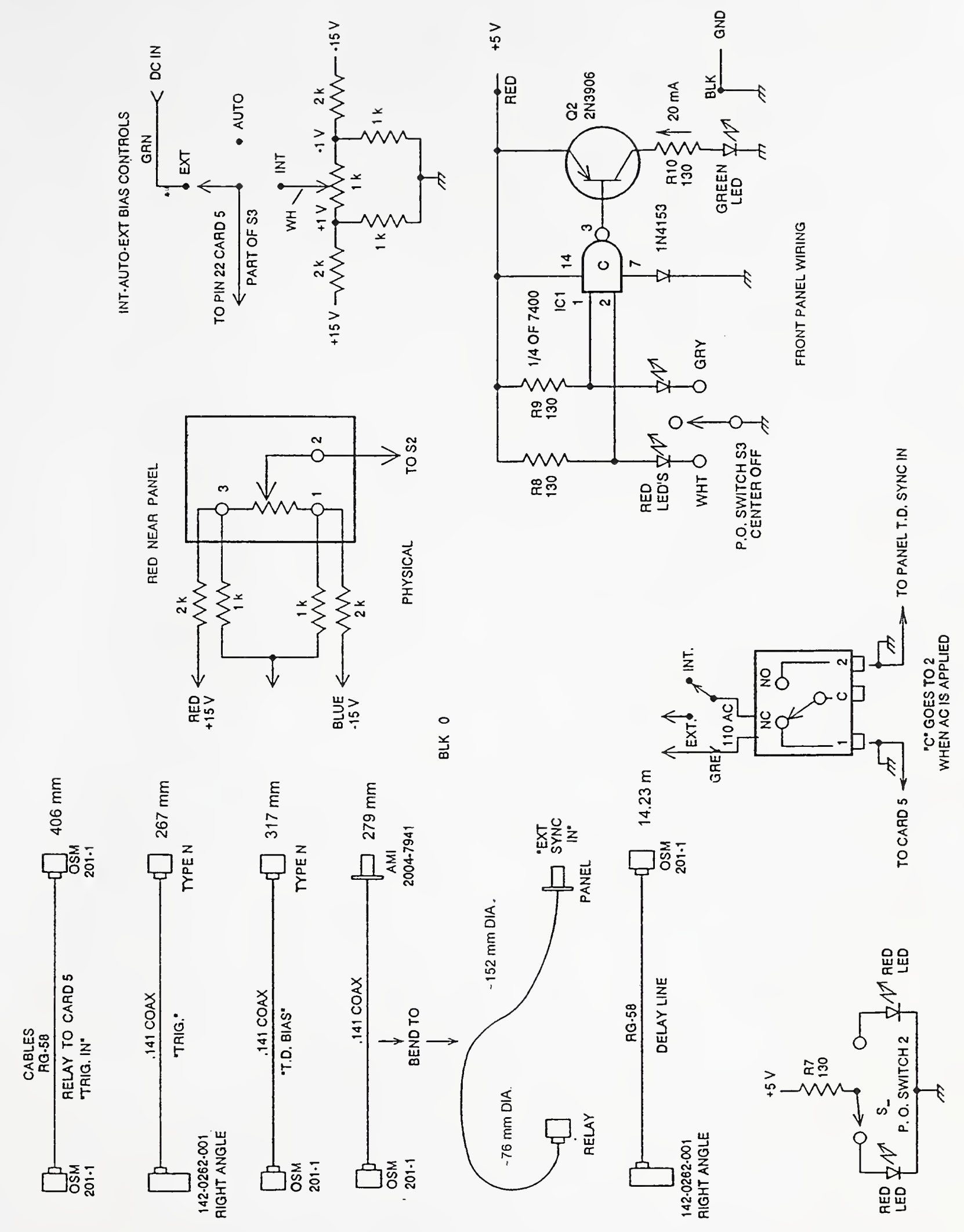

Automatic TD Bias Supply Front Panel Wiring 


